ESAIM: M2AN 55 (2021) 1-36

https://doi.org/10.1051/m2an/2020067
ESAIM: Mathematical Modelling and Numerical Analysis

www.esaim-m2an.org

\title{
ASYMPTOTIC ANALYSIS FOR PERIODIC PERFORATED SHELLS
}

\author{
Georges Griso ${ }^{1}$, Michael HaucK ${ }^{2, *}$ and Julia Orlik ${ }^{2, *}$
}

\begin{abstract}
We consider a perforated half-cylindrical thin shell and investigate the limit behavior when the period and the thickness simultaneously go to zero. By using the decomposition of shell displacements presented in Griso [JMPA 89 (2008) 199-223] we obtain a priori estimates. With the unfolding and rescaling operator we transform the problem to a reference configuration. In the end this yields a homogenized limit problem for the shell.
\end{abstract}

Mathematics Subject Classification. 35B27, 74Q05, 74K25, 74B05.

Received November 6, 2019. Accepted September 3, 2020.

\section{INTRODUCTION}

We consider a thin heterogeneous half-cylindrical shell with an in-plane periodic porous structure, whereby the periodicity $\varepsilon$ is of the same order as the shell's thickness $2 \delta$ and small compared to its in-plane surface size. This paper provides an analysis for homogenization and dimension reduction of the shell. We want to point out that both tasks are performed simultaneously, where $\lim _{(\varepsilon, \delta) \rightarrow(0,0)} \frac{\delta}{\varepsilon} \rightarrow \kappa \in(0, \infty)$. This is necessary since homogenization and dimension reduction usually do not commute as it was shown e.g. in [5]. The presented approach via the rescaling-unfolding operator is closely related to the one given in Chapter 11 of [10] for plates and for heterogeneous beams in [15], but new in the context of a linear elastic shell. There are various different homogenization techniques, as for example asymptotic expansions presented in [1,21], via Gamma-convergence in [11] and the two-scale convergence introduced in [18]. Although, the homogenization of plates and shells is in focus of interest of some other well-known research groups, our approach provides all the estimates and gives the limit not in terms of energy bounds, but yields a computational tool for the effective shell coefficients on its exact topology, which is important for applications.

Dimension reduction and homogenization of elastic plates via an asymptotic expansion technique can be found in [20]. Dimension reduction and homogenization of a shell for the diffusion problem in the sense of two-scale convergence was presented in [19], where it was shown that the curvature does not enter the homogenized model. Moreover, the homogenization for piezoelectric perforated shells without dimension reduction was presented in [12]. We want to mention, that the dimension reduction of a homogeneous shell was analyzed in $[3,8,17]$. For some notions of classical results in functional analysis we refer to $[22,23]$.

Keywords and phrases. Homogenization, dimension reduction, linear elasticity, shell, perforated domains.

1 Sorbonne Université, CNRS, Université de Paris, Laboratoire Jacques-Louis Lions (LJLL), F-75005 Paris, France.

2 Fraunhofer ITWM, Fraunhofer-Platz 1, 67663 Kaiserslautern, Germany.

*Corresponding author: michael.hauck91@gmail.com, julia.orlik@itwm.fraunhofer.de 
In our analysis, we begin with a general extension technique (based on results developed in [14]) for displacements acting on a perforated shell made of a network of thin cylinders to the full shell domain (see Prop. 1.1). The result is crucial for the following analysis. We assume that the shell is fixed along the lateral boundary and continue with a decomposition approach for thin structures introduced in [14]. This decomposition technique allows to represent any $H^{1}$-displacement of the shell through the displacement of its mid-surface, the rotations of the small segments orthogonal to the mid-surface and a warping term which takes into account the deformation of these small segments. This leads to Korn inequalities and estimates for each term of the decomposition.

In Sections 2-4, the rescaling and unfolding operators are introduced and the strain tensor is considered on a reference domain. To describe the limit behavior of the strain tensor of the whole shell, we decompose the mid-surface displacement as the sum of an inextentional displacement and an extentional (see Sect. 5). They correspond respectively to the bending and to a generalization of the membrane displacements for a plate. This decomposition has been introduced in [3]. A similar approach has been developed for curved beam in $[4,13]$.

Section 6.1 presents assumptions on forces in the right-hand side, rescaling them in a detailed manner.

At the end the limit problem is discussed. Especially Section 8.1 is important for applications, where the variational problem for an anisotropic homogenized shell is presented together with an expression to compute its effective coefficients via 6 auxiliary cell problems. The limit extensional and inextensional displacements $\left(\mathcal{U}_{E} \in \mathbb{D}_{E}, \mathcal{U} \in \mathbb{D}_{I}\right)$ solve the homogenized problem

$$
\begin{aligned}
\int_{\omega}\left[a_{\alpha \beta \alpha^{\prime} \beta^{\prime}} e_{\alpha \beta}\left(\mathcal{U}_{E}\right) e_{\alpha^{\prime} \beta^{\prime}}\left(\mathcal{V}_{E}\right)+b_{\alpha \beta \alpha^{\prime} \beta^{\prime}}^{\text {hom }}\left(e_{\alpha \beta}\left(\mathcal{U}_{E}\right) \frac{\partial}{\partial s_{\beta^{\prime}}}\left(\frac{\partial \mathcal{V}}{\partial s_{\alpha^{\prime}}} \mathbf{n}\right)\right.\right. \\
\left.\left.\quad+\frac{\partial}{\partial s_{\beta}}\left(\frac{\partial \mathcal{U}}{\partial s_{\alpha}} \mathbf{n}\right) e_{\alpha^{\prime} \beta^{\prime}}\left(\mathcal{V}_{E}\right)\right)+c_{\alpha \beta \alpha^{\prime} \beta^{\prime}}^{\text {hom }} \frac{\partial}{\partial s_{\beta}}\left(\frac{\partial \mathcal{U}}{\partial s_{\alpha}} \mathbf{n}\right) \frac{\partial}{\partial s_{\beta^{\prime}}}\left(\frac{\partial \mathcal{V}}{\partial s_{\alpha^{\prime}}} \mathbf{n}\right)\right] \mathrm{d} s \\
=\frac{\left|Y^{\prime *}\right|}{\left|Y^{\prime}\right|}\left(\int_{\omega}\left(f \cdot \mathcal{V}+\frac{\kappa^{2}}{3 a} g_{\alpha} \mathcal{V}_{\alpha}-\frac{\kappa^{2}}{3} g_{\alpha} \frac{\partial \mathcal{V}}{\partial s_{\alpha}} \mathbf{n}\right) \mathrm{d} s+\left\langle F, \mathcal{V}_{E}\right\rangle\right), \quad \forall\left(\mathcal{V}_{E}, \mathcal{V}\right) \in \mathbb{D}_{E} \times \mathbb{D}_{I}
\end{aligned}
$$

In Section 9 we focus on the effects of the boundary conditions in our model, which play an important role. Especially, if we fix the shell's curved ends we obtain a membrane dominated limit equation. In that case clamping the lateral boundary does not change the model.

\subsection{Geometrical setting}

We consider a cylindrical half-shell with constant radius $a$. We assume that our shell consists of a periodic structure with a periodicity cell of size $\varepsilon$ in its mid-plane, and is of thickness $2 \delta$, with $\delta=\kappa \varepsilon \in\left(0, \delta_{0}\right], \delta_{0}=a / 3$, where $\kappa$ is a strictly positive fixed constant.

Let $Y^{\prime}$ be a bounded domain in $\mathbb{R}^{2}$ having the paving property with respect to an additive subgroup $\mathbf{G} \doteq \mathbf{p}_{1} \mathbb{Z} \oplus \mathbf{p}_{2} \mathbb{Z}$ of $\mathbb{R}^{2}$ of dimension 2 and let $T$ be an open set such that $\bar{T} \subset Y^{\prime}$ (Fig. 1 gives an example of such a cell $Y^{\prime}$ ). We assume the boundary of $T$ to be Lipschitz and for simplicity we also assume $T$ connected. Denote

$$
\omega \doteq(0, a \pi) \times(0, l), \quad Y \doteq Y^{\prime} \times(-\kappa, \kappa), \quad Y^{\prime *} \doteq Y^{\prime} \backslash \bar{T}, \quad Y^{*} \doteq Y^{*} \times(-\kappa, \kappa) .
$$

In the periodic setting a.e. $s^{\prime} \in \mathbb{R}^{2}$ can be decomposed as

$$
s^{\prime}=\varepsilon\left[\frac{s^{\prime}}{\varepsilon}\right]_{Y^{\prime}}+\varepsilon\left\{\frac{s^{\prime}}{\varepsilon}\right\}_{Y^{\prime}}
$$

where $[\cdot]_{Y^{\prime}}$ belongs to $\mathbf{G}$ and $\{\cdot\}_{Y^{\prime}}$ to $Y^{\prime}$.

Set

$$
\Xi_{\varepsilon}=\left\{\xi \in \mathbf{G} \mid \varepsilon \xi+\varepsilon Y^{\prime} \subset \omega\right\}, \quad \widehat{\omega}_{\varepsilon}=\text { interior }\left\{\bigcup_{\xi \in \Xi_{\varepsilon}}\left(\varepsilon \xi+\varepsilon \overline{Y^{\prime}}\right)\right\}, \quad \Lambda_{\varepsilon}=\omega \backslash \overline{\widehat{\omega}_{\varepsilon}} .
$$



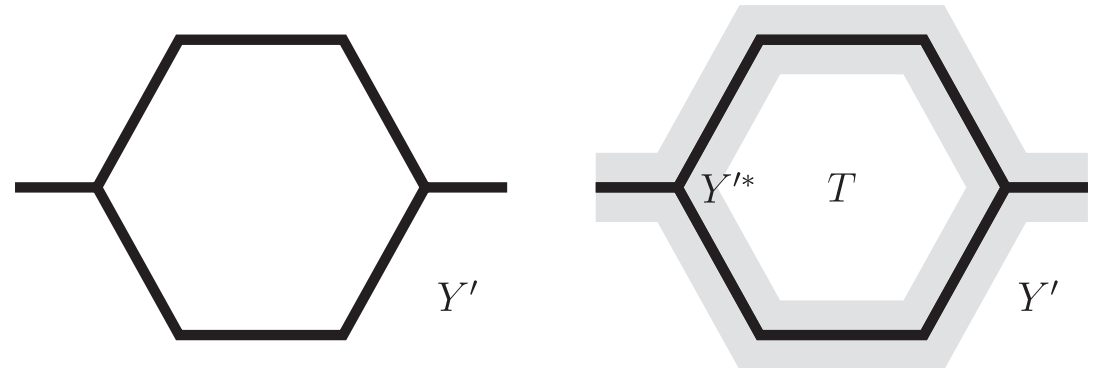

Figure 1. Cell $Y^{\prime}$ and the perforated domain $Y^{\prime *}$.

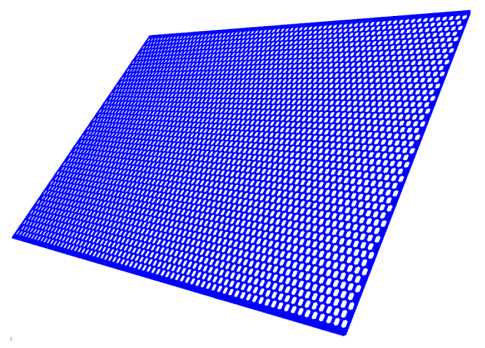

(a) Plane domain $\omega_{\varepsilon}^{*}$ with periodic hexagonal holes

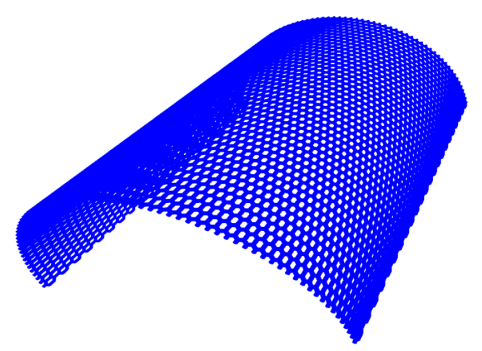

(b) Shell $\mathcal{Q}_{\varepsilon}^{*}$ with periodic holes

Figure 2. Periodic perforated plane domain transformed to a periodic shell. (a) Plane domain $\omega_{\varepsilon}^{*}$ with periodic hexagonal holes. (b) Shell $\mathcal{Q}_{\varepsilon}^{*}$ with periodic holes.

Let us also introduce some notations for the unions of all holes

$$
T_{\varepsilon} \doteq\left\{x \in \widehat{\omega}_{\varepsilon} \mid\left\{\frac{x}{\varepsilon}\right\}_{Y^{\prime}} \in T\right\}, \quad \omega_{\varepsilon}^{*}=\omega \backslash \overline{T_{\varepsilon}}, \quad \widehat{\omega}_{\varepsilon}^{*}=\widehat{\omega}_{\varepsilon} \backslash \overline{T_{\varepsilon}} .
$$

Consider the injective mapping $\phi: \bar{\omega} \rightarrow \mathbb{R}^{3}$ defined as

$$
\phi\left(s_{1}, s_{2}\right)=\left(\begin{array}{c}
s_{2} \\
a \cos \left(\frac{s_{1}}{a}\right) \\
a \sin \left(\frac{s_{1}}{a}\right)
\end{array}\right), \quad\left(s_{1}, s_{2}\right) \in \bar{\omega},
$$

and denote by $S=\phi(\bar{\omega})$ the mid-surface of the whole shell (without the holes). Furthermore, we introduce the vectors

$$
\mathbf{t}_{1}=\left(\begin{array}{c}
0 \\
-\sin \left(\frac{s_{1}}{a}\right) \\
\cos \left(\frac{s_{1}}{a}\right)
\end{array}\right), \quad \mathbf{t}_{2}=\left(\begin{array}{l}
1 \\
0 \\
0
\end{array}\right), \quad \mathbf{n}=\frac{\mathbf{t}_{1} \wedge \mathbf{t}_{2}}{\left\|\mathbf{t}_{1} \wedge \mathbf{t}_{2}\right\|_{2}}=\left(\begin{array}{c}
0 \\
\cos \left(\frac{s_{1}}{a}\right) \\
\sin \left(\frac{s_{1}}{a}\right)
\end{array}\right) .
$$

Obviously, $\mathbf{t}_{1}$ and $\mathbf{t}_{2}$ are linearly independent and are tangential vectors to the surface $S$ (Fig. 2).

Denote

- $\Omega_{\varepsilon}=\omega \times(-\kappa \varepsilon, \kappa \varepsilon), \Omega_{\varepsilon}^{*}=\omega_{\varepsilon}^{*} \times(-\kappa \varepsilon, \kappa \varepsilon)$,

- $\mathcal{Q}_{\varepsilon}^{*}=\Phi\left(\Omega_{\varepsilon}^{*}\right)$ the perforated shell,

- $\mathcal{Q}_{\varepsilon}=\Phi\left(\Omega_{\varepsilon}\right)$ the shell without the holes 
where $\Phi: \bar{\Omega}_{\varepsilon} \subset \mathbb{R}^{3} \rightarrow \mathbb{R}^{3}$ is given by

$$
\Phi(s)=\phi\left(s_{1}, s_{2}\right)+s_{3} \mathbf{n}\left(s_{1}, s_{2}\right), \quad s=\left(s_{1}, s_{2}, s_{3}\right) \in \bar{\Omega}_{\varepsilon} .
$$

We easily check that if $\delta=\kappa \varepsilon \in\left(0, \delta_{0}\right]$ the map $\Phi$ from $\bar{\Omega}_{\varepsilon}$ onto $\overline{\mathcal{Q}_{\varepsilon}}$ is a $C^{1}$-diffeomorphism. That means we have

$$
c_{0} \leq\left\|\nabla_{s} \Phi\right\|_{L^{\infty}\left(\Omega_{\varepsilon}\right)} \leq c_{1} \quad \text { and } \quad c_{0} \leq\left\|\nabla_{x} \Phi^{-1}\right\|_{L^{\infty}\left(\mathcal{Q}_{\varepsilon}\right)} \leq c_{1} .
$$

The constants do not depend on $\varepsilon$.

We denote by $x$ the running point of the shell while $s$, s.t. $\Phi(s)=x$, is the running point in the reference domain. A function $u$ defined on $\mathcal{Q}_{\varepsilon}\left(\right.$ resp. $\left.\mathcal{Q}_{\varepsilon}^{*}\right)$ can also be considered as a function defined on $\Omega_{\varepsilon}\left(\right.$ resp. $\left.\Omega_{\varepsilon}^{*}\right)$ which we also denote by $u$.

Proposition 1.1. There exists an extension operator $\mathcal{P}_{\varepsilon}$ from $H^{1}\left(\mathcal{Q}_{\varepsilon}^{*}\right)^{3}$ into $H^{1}\left(\mathcal{Q}_{\varepsilon}\right)^{3}$ satisfying for all $u \in$ $H^{1}\left(\mathcal{Q}_{\varepsilon}^{*}\right)^{3}$

$$
\mathcal{P}_{\varepsilon}(u)_{\mid \mathcal{Q}_{\varepsilon}^{*}}=u, \quad\left\|e\left(\mathcal{P}_{\varepsilon}(u)\right)\right\|_{L^{2}\left(\mathcal{Q}_{\varepsilon}\right)} \leq C\|e(u)\|_{L^{2}\left(\mathcal{Q}_{\varepsilon}^{*}\right)} .
$$

The constant does not depend on $\varepsilon$.

The proof of Proposition 1.1 has been moved to the Appendix A.

Set $\gamma_{0}=\{0\} \times[0, l] \cup\{\pi\} \times[0, l] \subset \partial \omega$. The part $\Gamma_{0, \varepsilon}=\Phi\left(\gamma_{0} \times(-\kappa \varepsilon, \kappa \varepsilon)\right)$ of the lateral boundary of the shell is clamped. The complementary of $\Gamma_{0, \varepsilon}$ in the lateral boundary of the shell is a free boundary.

From now on, any displacement $u$ belonging to $H^{1}\left(\mathcal{Q}_{\varepsilon}^{*}\right)^{3}$ will be extended to a displacement belonging to $H^{1}\left(\mathcal{Q}_{\varepsilon}\right)^{3}$. We will always denote by $u$ the extended displacement, which will satisfy (1.6). This displacement (still denoted $u$ ) could also be considered as an element of $H^{1}\left(\Omega_{\varepsilon}^{*}\right)^{3}$ or $H^{1}\left(\Omega_{\varepsilon}\right)^{3}$.

\subsection{Decomposition of shell displacements}

In this section we introduce a decomposition for every displacement $u$ of the shell $\mathcal{Q}_{\varepsilon}^{*}$ as it was shown in [14].

Definition 1.2. An elementary displacement $U_{e}$ associated to $u \in H^{1}\left(\Omega_{\varepsilon}\right)^{3}$ is given by

$$
U_{e}=\mathcal{U}\left(s_{1}, s_{2}\right)+s_{3} \mathcal{R}\left(s_{1}, s_{2}\right),
$$

where $(\alpha \in\{1,2\})$

$$
\mathcal{U}=\frac{1}{2 \kappa \varepsilon} \int_{-\kappa \varepsilon}^{\kappa \varepsilon} u\left(\cdot, s_{3}\right) \mathrm{d} s_{3}, \quad \mathcal{R}_{\alpha}=\frac{3}{2(\kappa \varepsilon)^{3}} \int_{-\kappa \varepsilon}^{\kappa \varepsilon} s_{3} u\left(\cdot, s_{3}\right) \cdot \mathbf{t}_{\alpha} \mathrm{d} s_{3}, \quad \mathcal{R}_{3}=0 \quad \text { a.e. } \quad \text { in } \quad \omega .
$$

Moreover, we have that $\mathcal{U}=\left(\mathcal{U}_{1}, \mathcal{U}_{2}, \mathcal{U}_{3}\right) \in H^{1}(\omega)^{3}$ and $\mathcal{R}=\left(\mathcal{R}_{1}, \mathcal{R}_{2}\right) \in H^{1}(\omega)^{2}$. Every displacement $u$ is then decomposed as

$$
U_{e}\left(\cdot, s_{3}\right)=\sum_{\alpha=1}^{2}\left(\mathcal{U}_{\alpha}+s_{3} \mathcal{R}_{\alpha}\right) \mathbf{t}_{\alpha}+\mathcal{U}_{3} \mathbf{n}, \quad u=U_{e}+\bar{u},
$$

where $\bar{u} \in H^{1}\left(\Omega_{\varepsilon}\right)^{3}$ is a residual displacement called warping.

Denote

$$
\begin{aligned}
V_{\varepsilon} & \doteq\left\{v \in H^{1}\left(\mathcal{Q}_{\varepsilon}\right)^{3} \mid v=0 \quad \text { on } \quad \Gamma_{0, \varepsilon}\right\}, \quad V_{\varepsilon}^{*} \doteq\left\{v \in H^{1}\left(\mathcal{Q}_{\varepsilon}^{*}\right)^{3} \mid v=0 \quad \text { on } \quad \Gamma_{0, \varepsilon}\right\}, \\
H_{\Gamma_{0}}^{1}(\omega) & \doteq\left\{\Phi \in H^{1}(\omega) \mid \Phi=0 \quad \text { on } \quad \Gamma_{0}\right\} .
\end{aligned}
$$

One has

$$
\mathcal{U} \in H_{\Gamma_{0}}^{1}(\omega)^{3}, \quad \mathcal{R} \in H_{\Gamma_{0}}^{1}(\omega)^{2}, \quad \bar{u} \in V_{\varepsilon} .
$$


Remark 1.3. The warping $\bar{u}$ fulfills the following properties

$$
\int_{-\kappa \varepsilon}^{\kappa \varepsilon} \bar{u}\left(\cdot, s_{3}\right) \mathrm{d} s_{3}=0, \quad \int_{-\kappa \varepsilon}^{\kappa \varepsilon} s_{3} \bar{u}\left(\cdot, s_{3}\right) \cdot \mathbf{t}_{\alpha} \mathrm{d} s_{3}=0 .
$$

For $\mathcal{U}$ and $\mathcal{R}$ holds

$$
\mathcal{U}=\mathcal{U}_{1} \mathbf{t}_{1}+\mathcal{U}_{2} \mathbf{t}_{2}+\mathcal{U}_{3} \mathbf{n}, \quad \mathcal{R}=\mathcal{R}_{1} \mathbf{t}_{1}+\mathcal{R}_{2} \mathbf{t}_{2} .
$$

In the next step we want to establish the strain tensor in the cylindrical coordinates. The derivatives of the elementary displacement $U_{e}$ are calculated using

$$
\begin{aligned}
\frac{\partial \mathcal{U}}{\partial s_{1}} & =\frac{\partial \mathcal{U}_{1}}{\partial s_{1}} \mathbf{t}_{1}-\frac{1}{a} \mathcal{U}_{1} \mathbf{n}+\frac{\partial \mathcal{U}_{2}}{\partial s_{1}} \mathbf{t}_{2}+\frac{\partial \mathcal{U}_{3}}{\partial s_{1}} \mathbf{n}+\frac{1}{a} \mathcal{U}_{3} \mathbf{t}_{1}, \\
\frac{\partial \mathcal{U}}{\partial s_{2}} & =\frac{\partial \mathcal{U}_{1}}{\partial s_{2}} \mathbf{t}_{1}+\frac{\partial \mathcal{U}_{2}}{\partial s_{2}} \mathbf{t}_{2}+\frac{\partial \mathcal{U}_{3}}{\partial s_{2}} \mathbf{n}
\end{aligned}
$$

and

$$
\begin{aligned}
& \frac{\partial \mathcal{R}}{\partial s_{1}}=\frac{\partial \mathcal{R}_{1}}{\partial s_{1}} \mathbf{t}_{1}-\frac{1}{a} \mathcal{R}_{1} \mathbf{n}+\frac{\partial \mathcal{R}_{2}}{\partial s_{1}} \mathbf{t}_{2}, \\
& \frac{\partial \mathcal{R}}{\partial s_{2}}=\frac{\partial \mathcal{R}_{1}}{\partial s_{2}} \mathbf{t}_{1}+\frac{\partial \mathcal{R}_{2}}{\partial s_{2}} \mathbf{t}_{2} .
\end{aligned}
$$

The strain tensor for a shell displacement $u \in H^{1}\left(\mathcal{Q}_{\varepsilon}\right)$ is given by

$$
e_{x}(u)=\frac{\nabla_{x} u+\left(\nabla_{x} u\right)^{T}}{2} .
$$

A small computation yields, that $\nabla_{s}$ in the coordinates of the reference domain is given by

$$
\nabla_{s}=\nabla_{x} \nabla \Phi
$$

Furthermore, we still have that $e_{x}(u)$ is in the shell configuration. Therefore, we consider the transformation matrix $\left(\mathbf{t}_{1}\left|\mathbf{t}_{2}\right| \mathbf{n}\right)$ and transfer our strain matrix into the reference domain by

$$
\left(\mathbf{t}_{1}\left|\mathbf{t}_{2}\right| \mathbf{n}\right)^{T} e_{x}(u)\left(\mathbf{t}_{1}\left|\mathbf{t}_{2}\right| \mathbf{n}\right) .
$$

Definition 1.4. We define by $e(u)$ the strain tensor in the coordinates of the reference domain by

$$
e(u)=\left(\mathbf{t}_{1}\left|\mathbf{t}_{2}\right| \mathbf{n}\right)^{T} \frac{\nabla_{s} u(\nabla \Phi)^{-1}+\left(\nabla_{s} u(\nabla \Phi)^{-1}\right)^{T}}{2}\left(\mathbf{t}_{1}\left|\mathbf{t}_{2}\right| \mathbf{n}\right)
$$

Hence, we obtain

$$
\begin{aligned}
\left(\mathbf{t}_{1}\left|\mathbf{t}_{2}\right| \mathbf{n}\right)^{T} \nabla_{s} u(\nabla \Phi)^{-1}\left(\mathbf{t}_{1}\left|\mathbf{t}_{2}\right| \mathbf{n}\right) & =\left(\mathbf{t}_{1}\left|\mathbf{t}_{2}\right| \mathbf{n}\right)^{T} \nabla_{s} u\left(\begin{array}{ccc}
\frac{a}{a+s_{3}} & 0 & 0 \\
0 & 1 & 0 \\
0 & 0 & 1
\end{array}\right) \\
& =\left(\begin{array}{ccc}
\frac{a}{a+s_{3}} \frac{\partial u}{\partial s_{1}} \mathbf{t}_{1} & \frac{\partial u}{\partial s_{2}} \mathbf{t}_{1} & \frac{\partial u}{\partial s_{3}} \mathbf{t}_{1} \\
\frac{a}{a+s_{3}} \frac{\partial u}{\partial s_{1}} \mathbf{t}_{2} & \frac{\partial u}{\partial s_{2}} \mathbf{t}_{2} & \frac{\partial u}{\partial s_{3}} \mathbf{t}_{2} \\
\frac{a}{a+s_{3}} \frac{\partial u}{\partial s_{1}} \mathbf{n} & \frac{\partial u}{\partial s_{2}} \mathbf{n} & \frac{\partial u}{\partial s_{3}} \mathbf{n}
\end{array}\right),
\end{aligned}
$$


where

$$
\begin{aligned}
\frac{\partial u}{\partial s_{1}}= & \left(\frac{\partial \mathcal{U}_{1}}{\partial s_{1}}+s_{3} \frac{\partial \mathcal{R}_{1}}{\partial s_{1}}+\frac{\partial \bar{u}_{1}}{\partial s_{1}}+\frac{1}{a}\left(\mathcal{U}_{3}+\bar{u}_{3}\right)\right) \mathbf{t}_{1}+\left(\frac{\partial \mathcal{U}_{2}}{\partial s_{1}}+s_{3} \frac{\partial \mathcal{R}_{2}}{\partial s_{1}}+\frac{\partial \bar{u}_{2}}{\partial s_{1}}\right) \mathbf{t}_{2} \\
& +\left(\frac{\partial \mathcal{U}_{3}}{\partial s_{1}}+\frac{\partial \bar{u}_{3}}{\partial s_{1}}-\frac{1}{a}\left(\mathcal{U}_{1}+s_{3} \mathcal{R}_{1}+\bar{u}_{1}\right)\right) \mathbf{n}, \\
\frac{\partial u}{\partial s_{2}}= & \left(\frac{\partial \mathcal{U}_{1}}{\partial s_{2}}+s_{3} \frac{\partial \mathcal{R}_{1}}{\partial s_{2}}+\frac{\partial \bar{u}_{1}}{\partial s_{2}}\right) \mathbf{t}_{1}+\left(\frac{\partial \mathcal{U}_{2}}{\partial s_{2}}+s_{3} \frac{\partial \mathcal{R}_{2}}{\partial s_{2}}+\frac{\partial \bar{u}_{2}}{\partial s_{2}}\right) \mathbf{t}_{2}+\left(\frac{\partial \mathcal{U}_{3}}{\partial s_{2}}+\frac{\partial \bar{u}_{3}}{\partial s_{2}}\right) \mathbf{n}, \\
\frac{\partial u}{\partial s_{3}}= & \left(\mathcal{R}_{1}+\frac{\partial \bar{u}_{1}}{\partial s_{3}}\right) \mathbf{t}_{1}+\left(\mathcal{R}_{2}+\frac{\partial \bar{u}_{2}}{\partial s_{3}}\right) \mathbf{t}_{2}+\frac{\partial \bar{u}_{3}}{\partial s_{3}} \mathbf{n} .
\end{aligned}
$$

We get for the strain tensor $e(u)$ of a displacement $u \in V_{\varepsilon}$ the following components:

$$
\begin{aligned}
e_{11}(u)= & \frac{a}{a+s_{3}}\left[\left(\frac{\partial \mathcal{U}_{1}}{\partial s_{1}}+\frac{1}{a} \mathcal{U}_{3}\right)+s_{3} \frac{\partial \mathcal{R}_{1}}{\partial s_{1}}+\frac{\partial \bar{u}_{1}}{\partial s_{1}}+\frac{1}{a} \bar{u}_{3}\right], \\
e_{22}(u)= & \frac{\partial \mathcal{U}_{2}}{\partial s_{2}}+s_{3} \frac{\partial \mathcal{R}_{2}}{\partial s_{2}}+\frac{\partial \bar{u}_{2}}{\partial s_{2}}, \\
e_{12}(u)= & \frac{1}{2} \frac{a}{a+s_{3}}\left[\left(\frac{\partial \mathcal{U}_{2}}{\partial s_{1}}+\frac{\partial \mathcal{U}_{1}}{\partial s_{2}}\right)+s_{3}\left(\frac{\partial \mathcal{R}_{2}}{\partial s_{1}}+\frac{\partial \mathcal{R}_{1}}{\partial s_{2}}\right)+\frac{s_{3}}{a} \frac{\partial \mathcal{U}_{1}}{\partial s_{2}}+\frac{s_{3}^{2}}{a} \frac{\partial \mathcal{R}_{1}}{\partial s_{2}}\right. \\
& \left.+\frac{\partial \bar{u}_{2}}{\partial s_{1}}+\left(1+\frac{s_{3}}{a}\right) \frac{\partial \bar{u}_{1}}{\partial s_{2}}\right], \\
e_{13}(u)= & \frac{1}{2} \frac{a}{a+s_{3}}\left[\left(\frac{\partial \mathcal{U}_{3}}{\partial s_{1}}-\frac{1}{a} \mathcal{U}_{1}+\mathcal{R}_{1}\right)-\frac{1}{a} \bar{u}_{1}+\frac{\partial \bar{u}_{3}}{\partial s_{1}}+\left(1+\frac{s_{3}}{a}\right) \frac{\partial \bar{u}_{1}}{\partial s_{3}}\right], \\
e_{23}(u)= & \frac{1}{2}\left\{\left(\frac{\partial \mathcal{U}_{3}}{\partial s_{2}}+\mathcal{R}_{2}\right)+\frac{\partial \bar{u}_{3}}{\partial s_{2}}+\frac{\partial \bar{u}_{2}}{\partial s_{3}}\right\}, \quad e_{33}(u)=\frac{\partial \bar{u}_{3}}{\partial s_{3}} .
\end{aligned}
$$

Theorem 1.5. Let $u \in H^{1}\left(\mathcal{Q}_{\varepsilon}^{*}\right)^{3}$ and $(\mathcal{U}, \mathcal{R}, \bar{u})$ be the terms of its decomposition, then the following inequalities are satisfied:

$$
\begin{aligned}
\left\|e\left(U_{e}\right)\right\|_{L^{2}\left(\Omega_{\varepsilon}\right)} & \leq C\|e(u)\|_{L^{2}\left(\mathcal{Q}_{\varepsilon}^{*}\right)} \\
\|\bar{u}\|_{L^{2}\left(\mathcal{Q}_{\varepsilon}\right)} & \leq C \varepsilon\|e(u)\|_{L^{2}\left(\mathcal{Q}_{\varepsilon}^{*}\right)} \\
\|\nabla \bar{u}\|_{L^{2}\left(\mathcal{Q}_{\varepsilon}\right)} & \leq C\|e(u)\|_{L^{2}\left(\mathcal{Q}_{\varepsilon}^{*}\right)} .
\end{aligned}
$$

Proof. The proof is given in Theorem 4.1 of [14].

From [14] we also obtain the full estimates of $u$ and the components of the elementary displacement $U_{e}$.

Proposition 1.6. For every $u \in V_{\varepsilon}^{*}$

$$
\|u\|_{H^{1}\left(\Omega_{\varepsilon}\right)} \leq \frac{C}{\varepsilon}\|e(u)\|_{L^{2}\left(\mathcal{Q}_{\varepsilon}^{*}\right)}, \quad\|\mathcal{R}\|_{H^{1}(\omega)}+\|\mathcal{U}\|_{H^{1}(\omega)} \leq \frac{C}{\varepsilon^{3 / 2}}\|e(u)\|_{L^{2}\left(\mathcal{Q}_{\varepsilon}^{*}\right)} .
$$

The constants do not depend on $\varepsilon$.

From the expression of the strain tensor $e(u)$ one derives the following estimates:

Lemma 1.7. One has also the following estimates $\left((\alpha, \beta) \in\{1,2\}^{2}\right)$ :

$$
\left\|\frac{\partial \mathcal{U}}{\partial s_{\alpha}} \cdot \boldsymbol{t}_{\beta}+\frac{\partial \mathcal{U}}{\partial s_{\beta}} \cdot \boldsymbol{t}_{\alpha}\right\|_{L^{2}(\omega)}+\left\|\frac{\partial \mathcal{U}}{\partial s_{\alpha}} \cdot \boldsymbol{n}+\mathcal{R} \cdot \boldsymbol{t}_{\alpha}\right\|_{L^{2}(\omega)} \leq \frac{C}{\varepsilon^{1 / 2}}\|e(u)\|_{L^{2}\left(\mathcal{Q}_{\varepsilon}^{*}\right)} .
$$

The constant does not depend on $\varepsilon$. 
Proof. We will only show that

$$
\left\|\frac{\partial \mathcal{U}_{2}}{\partial s_{1}}+\frac{\partial \mathcal{U}_{1}}{\partial s_{2}}\right\|_{L^{2}(\omega)} \leq \frac{C}{\varepsilon^{1 / 2}}\|e(u)\|_{L^{2}\left(\mathcal{Q}_{\varepsilon}^{*}\right)},
$$

since the other inequalities follow in the same way.

First observe that $\frac{a}{a+s_{3}}$ is uniformly bounded. Then, we start with the expression of $e_{12}(u)$ given by (1.18). Due to (1.19) and (1.20) we obtain

$$
\int_{\Omega_{\varepsilon}}\left[\left(\frac{\partial \mathcal{U}_{2}}{\partial s_{1}}+\frac{\partial \mathcal{U}_{1}}{\partial s_{2}}\right)+s_{3}\left(\frac{\partial \mathcal{R}_{2}}{\partial s_{1}}+\frac{\partial \mathcal{R}_{1}}{\partial s_{2}}\right)+\frac{s_{3}}{a} \frac{\partial \mathcal{U}_{1}}{\partial s_{2}}+\frac{s_{3}^{2}}{a} \frac{\partial \mathcal{R}_{1}}{\partial s_{2}}\right]^{2} \mathrm{~d} s \leq C\|e(u)\|_{L^{2}\left(\mathcal{Q}_{\varepsilon}^{*}\right)}^{2} .
$$

Hence, using the estimates (1.21)

$$
\varepsilon \int_{\omega}\left(\frac{\partial \mathcal{U}_{2}}{\partial s_{1}}+\frac{\partial \mathcal{U}_{1}}{\partial s_{2}}\right)^{2} \mathrm{~d} s \leq C\|e(u)\|_{L^{2}\left(\mathcal{Q}_{\varepsilon}^{*}\right)}^{2},
$$

which proves the inequality (1.23).

\section{The RESCAling Operator $\mathfrak{T}_{\varepsilon}$}

From now on we consider the reference domain

$$
\Omega=\omega \times(-\kappa, \kappa)
$$

and we rescale the shell in its $s_{3}$ direction.

Definition 2.1. Given a measurable function $\Psi$ on $\Omega_{\varepsilon}$, we define the measurable function $\mathfrak{T}_{\varepsilon}(\Psi)$ on $\Omega$ as

$$
\mathfrak{T}_{\varepsilon}(\Psi)\left(s_{1}, s_{2}, y_{3}\right)=\Psi\left(s_{1}, s_{2}, \varepsilon y_{3}\right), \quad \text { for } \quad \text { a.e. } \quad\left(s_{1}, s_{2}, y_{3}\right) \in \Omega .
$$

Lemma 2.2. One has for every $\Psi \in L^{2}\left(\Omega_{\varepsilon}\right)$ and for the warping $\bar{u}$

$$
\begin{aligned}
\left\|\mathfrak{T}_{\varepsilon}(\Psi)\right\|_{L^{2}(\Omega)} & \leq C \varepsilon^{-1 / 2}\|\Psi\|_{L^{2}\left(\Omega_{\varepsilon}\right)}, \quad\left\|\mathfrak{T}_{\varepsilon}(\bar{u})\right\|_{L^{2}(\Omega)} \leq C \varepsilon^{1 / 2}\|e(u)\|_{L^{2}\left(\mathcal{Q}_{\varepsilon}^{*}\right)}, \\
\left\|\frac{\partial \mathfrak{T}_{\varepsilon}(\bar{u})}{\partial s_{\alpha}}\right\|_{L^{2}(\Omega)} & \leq C \varepsilon^{-1 / 2}\|e(u)\|_{L^{2}\left(\mathcal{Q}_{\varepsilon}^{*}\right)}, \quad\left\|\frac{\partial \mathfrak{T}_{\varepsilon}(\bar{u})}{\partial y_{3}}\right\|_{L^{2}(\Omega)} \leq C \varepsilon^{1 / 2}\|e(u)\|_{L^{2}\left(\mathcal{Q}_{\varepsilon}^{*}\right)} .
\end{aligned}
$$

\section{Asymptotic Behavior OF THE STRAin TENSOR}

Lemma 3.1. Let $\left\{u_{\varepsilon}\right\}_{\varepsilon}$ be a sequence of displacements belonging to $V_{\varepsilon}^{*}$ and satisfying ${ }^{3}$

$$
\left\|e\left(u_{\varepsilon}\right)\right\|_{L^{2}\left(\mathcal{Q}_{\varepsilon}^{*}\right)} \leq C \varepsilon^{3 / 2}
$$

with a constant independent of $\varepsilon$.

There exists a subsequence (still denoted $\varepsilon$ ) and $\mathcal{U} \in H_{\Gamma_{0}}^{1}(\omega)^{3}, \mathcal{R} \in H_{\Gamma_{0}}^{1}(\omega)^{2}, \mathcal{Z}_{\alpha \beta} \in L^{2}(\omega), \mathcal{Z}_{\alpha 3} \in L^{2}(\omega)$ and $\bar{u} \in L^{2}\left(\omega ; H^{1}(-\kappa, \kappa)\right)^{3}$ satisfying

$$
\int_{-\kappa}^{\kappa} \bar{u}\left(\cdot, y_{3}\right) \mathrm{d} y_{3}=0, \quad \int_{-\kappa}^{\kappa} y_{3} \bar{u}_{\alpha}\left(\cdot, y_{3}\right) \mathrm{d} y_{3}=0 \quad \text { a.e. } \quad \text { in } \quad \omega
$$

\footnotetext{
${ }^{3}$ Or equivalently $\left\|e\left(u_{\varepsilon}\right)\right\|_{L^{2}\left(\mathcal{Q}_{\varepsilon}\right)} \leq C \varepsilon^{3 / 2}$ since the displacements are extended to the whole shell.
} 
such that

$$
\begin{aligned}
& \mathcal{U}_{\varepsilon} \longrightarrow \mathcal{U} \quad \text { strongly in } H_{\Gamma_{0}}^{1}(\omega)^{3}, \quad \mathcal{R}_{\varepsilon, \alpha} \rightarrow \mathcal{R}_{\alpha} \quad \text { weakly in } H_{\Gamma_{0}}^{1}(\omega), \\
& \frac{1}{\varepsilon}\left(\frac{\partial \mathcal{U}_{\varepsilon}}{\partial s_{\alpha}} \cdot \boldsymbol{t}_{\beta}+\frac{\partial \mathcal{U}_{\varepsilon}}{\partial s_{\beta}} \cdot \boldsymbol{t}_{\alpha}\right) \rightarrow \mathcal{Z}_{\alpha \beta} \quad \text { weakly in } L^{2}(\omega), \\
& \frac{1}{\varepsilon}\left(\frac{\partial \mathcal{U}_{\varepsilon}}{\partial s_{\alpha}} \cdot \boldsymbol{n}+\mathcal{R}_{\varepsilon} \cdot \boldsymbol{t}_{\alpha}\right) \rightarrow \mathcal{Z}_{\alpha 3} \quad \text { weakly in } L^{2}(\omega), \\
& \frac{1}{\varepsilon^{2}} \mathfrak{T}_{\varepsilon}\left(\bar{u}_{\varepsilon}\right) \rightarrow \bar{u} \quad \text { weakly in } L^{2}\left(\omega ; H^{1}(-\kappa, \kappa)\right)^{3}, \\
& \frac{1}{\varepsilon} \mathfrak{T}_{\varepsilon}\left(\frac{\partial \bar{u}_{\varepsilon}}{\partial s_{\alpha}}\right)=\frac{1}{\varepsilon} \frac{\partial}{\partial s_{\alpha}} \mathfrak{T}_{\varepsilon}\left(\bar{u}_{\varepsilon}\right) \rightarrow 0 \quad \text { weakly in } L^{2}(\omega \times(-\kappa, \kappa))^{3}, \\
& \frac{1}{\varepsilon} \mathfrak{T}_{\varepsilon}\left(e\left(u_{\varepsilon}\right)\right) \rightarrow \mathcal{E}(\mathcal{U}, \mathcal{Z}, \bar{u}) \quad \text { weakly } \quad \text { in } L^{2}(\omega)^{3 \times 3} .
\end{aligned}
$$

Moreover, one has

$$
\frac{\partial \mathcal{U}_{3}}{\partial s_{1}}-\frac{1}{a} \mathcal{U}_{1}+\mathcal{R}_{1}=0, \quad \frac{\partial \mathcal{U}_{3}}{\partial s_{2}}+\mathcal{R}_{2}=0
$$

Proof. We start with the weak limits; they are the consequences of (1.21).

$$
\mathcal{U}_{\varepsilon} \rightarrow \mathcal{U} \quad \text { weakly in } H_{\Gamma_{0}}^{1}(\omega)^{3}, \quad \mathcal{R}_{\varepsilon} \rightarrow \mathcal{R} \quad \text { weakly in } H_{\Gamma_{0}}^{1}(\omega)^{2} .
$$

The results in (3.2) $)_{5,6}$ follow from Lemma 2.2 and equation (1.19).

Both convergences $(3.2)_{3,4}$ follow directly from Lemma 1.7 .

Now we prove

$$
\mathcal{U}_{\varepsilon, 3} \longrightarrow \mathcal{U}_{3} \quad \text { strongly } \quad \text { in } \quad H_{\Gamma_{0}}^{1}(\omega) .
$$

By the Sobolev embedding and convergences (3.3), one has

$$
\mathcal{U}_{\varepsilon} \longrightarrow \mathcal{U} \text { strongly in } L^{2}(\omega)^{3}, \quad \mathcal{R}_{\varepsilon} \longrightarrow \mathcal{R} \text { strongly in } L^{2}(\omega)^{2} .
$$

Besides, from estimate $(1.22)_{2}$, one obtains

$$
\begin{aligned}
& \frac{\partial \mathcal{U}_{\varepsilon, 3}}{\partial s_{1}}-\frac{1}{a} \mathcal{U}_{\varepsilon, 1}+\mathcal{R}_{\varepsilon, 1} \longrightarrow 0 \quad \text { strongly } \quad \text { in } \quad L^{2}(\omega), \\
& \frac{\partial \mathcal{U}_{\varepsilon, 3}}{\partial s_{2}}+\mathcal{R}_{\varepsilon, 2} \longrightarrow 0 \quad \text { strongly } \quad \text { in } \quad L^{2}(\omega) .
\end{aligned}
$$

Hence $\nabla \mathcal{U}_{\varepsilon, 3}$ strongly converges to its limit in $L^{2}(\omega)^{2}$, which ends the proof of (3.4). That also proves the last equalities of the lemma.

Now, prove the strong convergences

$$
\mathcal{U}_{\varepsilon, \alpha} \longrightarrow \mathcal{U}_{\alpha} \quad \text { strongly } \quad \text { in } \quad H_{\Gamma_{0}}^{1}(\omega), \quad \alpha=1,2 .
$$

By estimate $(1.22)_{1}$ one immediately has

$$
\begin{aligned}
& \frac{\partial \mathcal{U}_{\varepsilon, 1}}{\partial s_{1}}+\frac{1}{a} \mathcal{U}_{\varepsilon, 3} \longrightarrow 0 \text { strongly in } L^{2}(\omega), \\
& \frac{\partial \mathcal{U}_{\varepsilon, 2}}{\partial s_{2}} \longrightarrow 0 \quad \text { strongly } \quad \text { in } L^{2}(\omega), \\
& \frac{\partial \mathcal{U}_{\varepsilon, 1}}{\partial s_{2}}+\frac{\partial \mathcal{U}_{\varepsilon, 2}}{\partial s_{1}} \longrightarrow 0 \text { strongly in } L^{2}(\omega) .
\end{aligned}
$$


Furthermore, from (3.5) and the above strong convergences, one obtains the strong convergence of the strain tensor of the displacement $\left(\mathcal{U}_{\varepsilon, 1}, \mathcal{U}_{\varepsilon, 2}\right)$ in $L^{2}(\omega)^{3}$. Since $\omega$ is a Lipschitz domain, this displacement strongly converges to its limit in $H_{\Gamma_{0}}^{1}(\omega)^{2}$. The elements of the limit strain tensor are then particularly given by $(\alpha \in$ $\{1,2\})$

$$
\begin{aligned}
& \frac{1}{\varepsilon} \mathfrak{T}_{\varepsilon}\left(e_{\alpha \alpha}\right) \rightarrow \mathcal{Z}_{\alpha \alpha}+y_{3} \frac{\partial \mathcal{R}_{\alpha}}{\partial s_{\alpha}}, \\
& \frac{1}{\varepsilon} \mathfrak{T}_{\varepsilon}\left(e_{12}\right) \rightarrow \frac{1}{2}\left\{\mathcal{Z}_{12}+\frac{y_{3}}{a} \frac{\partial \mathcal{U}_{1}}{\partial s_{2}}+y_{3} \frac{\partial \mathcal{R}_{2}}{\partial s_{1}}+y_{3} \frac{\partial \mathcal{R}_{1}}{\partial s_{2}}\right\}, \\
& \frac{1}{\varepsilon} \mathfrak{T}_{\varepsilon}\left(e_{\alpha 3}\right) \rightarrow \frac{1}{2}\left\{\mathcal{Z}_{\alpha 3}+\frac{\partial \bar{u}_{\alpha}}{\partial y_{3}}\right\}, \\
& \frac{1}{\varepsilon} \mathfrak{T}_{\varepsilon}\left(e_{33}\right) \rightarrow \frac{\partial \bar{u}_{3}}{\partial y_{3}} .
\end{aligned}
$$

Putting everything together we obtain the symmetric tensor

$$
\mathcal{E}(\mathcal{U}, \mathcal{Z}, \bar{u})=\left(\begin{array}{ccc}
\mathcal{Z}_{11}+\frac{y_{3}}{a} \frac{\partial \mathcal{U}_{1}}{\partial s_{1}}-y_{3} \frac{\partial^{2} \mathcal{U}_{3}}{\partial s_{1}^{2}} & \frac{1}{2} \mathcal{Z}_{12}+\frac{y_{3}}{a} \frac{\partial \mathcal{U}_{1}}{\partial s_{2}}-y_{3} \frac{\partial^{2} \mathcal{U}_{3}}{\partial s_{1} \partial s_{2}} & \frac{1}{2}\left(\mathcal{Z}_{13}+\frac{\partial \bar{u}_{1}}{\partial y_{3}}\right) \\
* & \mathcal{Z}_{22}-y_{3} \frac{\partial^{2} \mathcal{U}_{3}}{\partial s_{2}^{2}} & \frac{1}{2}\left(\mathcal{Z}_{23}+\frac{\partial \bar{u}_{2}}{\partial y_{3}}\right) \\
* & * & \frac{\partial \bar{u}_{3}}{\partial y_{3}}
\end{array}\right),
$$

which ends the proof of the lemma.

As a consequence of the estimates in Lemma 1.7 and the above lemma, one has a.e. in $\omega$ with $\mathcal{U}_{i} \in H_{\Gamma_{0}}^{1}(\omega)$ and $\mathcal{R}_{\alpha} \in H_{\Gamma_{0}}^{1}(\omega)$

$$
\frac{\partial \mathcal{U}}{\partial s_{\alpha}} \cdot \mathbf{t}_{\beta}+\frac{\partial \mathcal{U}}{\partial s_{\beta}} \cdot \mathbf{t}_{\alpha}=0, \quad \frac{\partial \mathcal{U}}{\partial s_{\alpha}} \cdot \mathbf{n}+\mathcal{R} \cdot \mathbf{t}_{\alpha}=0
$$

From the first equation in (3.6) we obtain for $(\alpha, \beta)=(2,2)$ that $\frac{\partial \mathcal{U}_{2}}{\partial s_{2}}=0$. Hence $\mathcal{U}_{2}$ does not depend on $s_{2}$, $\mathcal{U}_{2}=U_{2}\left(s_{1}\right)$ and due to the boundary conditions, one has $U_{2} \in H_{0}^{1}(0, a \pi)$.

With that we conclude for $(\alpha, \beta)=(1,2)$ that

$$
\mathcal{U}_{1}\left(s_{1}, s_{2}\right)=-s_{2} \frac{\mathrm{d} U_{2}}{\mathrm{~d} s_{1}}\left(s_{1}\right)+U_{1}\left(s_{1}\right)
$$

Since $\mathcal{U}_{1}$ belongs to $H_{\Gamma_{0}}^{1}(\omega)$, we get $U_{2} \in H_{0}^{2}(0, a \pi)$ and $U_{1} \in H_{0}^{1}(0, a \pi)$.

This yields for the last case, $(\alpha, \beta)=(1,1)$,

$$
\mathcal{U}_{3}\left(s_{1}, s_{2}\right)=a s_{2} \frac{\mathrm{d}^{2} U_{2}}{\mathrm{~d} s_{1}^{2}}-a \frac{\mathrm{d} U_{1}}{\mathrm{~d} s_{1}}\left(s_{1}\right)
$$

Since $\mathcal{U}_{3}$ belongs to $H_{\Gamma_{0}}^{1}(\omega)$, this implies at this step

$$
\begin{aligned}
U_{2} & \in H_{0}^{3}(0, a \pi), \quad U_{1} \in H_{0}^{2}(0, a \pi), \\
\text { and } \mathcal{U}\left(s_{1}, s_{2}\right) & =\left(-s_{2} \frac{\mathrm{d} U_{2}}{\mathrm{~d} s_{1}}\left(s_{1}\right)+U_{1}\left(s_{1}\right), U_{2}\left(s_{1}\right), a s_{2} \frac{\mathrm{d}^{2} U_{2}}{\mathrm{~d} s_{1}^{2}}-a \frac{\mathrm{d} U_{1}}{\mathrm{~d} s_{1}}\left(s_{1}\right)\right) .
\end{aligned}
$$

We now focus on the second equality given in (3.6), where we obtain with our expression for $\mathcal{U}$

$$
\mathcal{R}_{1}\left(s_{1}, s_{2}\right)=-s_{2}\left(\frac{1}{a} \frac{\mathrm{d} U_{2}}{\mathrm{~d} s_{1}}\left(s_{1}\right)+a \frac{\mathrm{d}^{3} U_{2}}{\mathrm{~d} s_{1}^{3}}\left(s_{1}\right)\right)+\frac{1}{a} U_{1}\left(s_{1}\right)+a \frac{\mathrm{d}^{2} U_{1}}{\mathrm{~d} s_{1}^{2}}\left(s_{1}\right), \quad \mathcal{R}_{2}\left(s_{1}, s_{2}\right)=-a \frac{\mathrm{d}^{2} U_{2}}{\mathrm{~d} s_{1}^{2}}\left(s_{1}\right) .
$$


Observe that due to the above conditions on $U_{2}, \mathcal{R}_{2}$ belongs to $H_{\Gamma_{0}}^{1}(\omega)$. Now, since $\mathcal{R}_{1}$ also belongs to $H_{\Gamma_{0}}^{1}(\omega)$, we finally obtain

$$
U_{2} \in H_{0}^{4}(0, a \pi), \quad U_{1} \in H_{0}^{3}(0, a \pi) .
$$

Thus

$$
\begin{array}{lll}
\mathcal{R}_{1} \in H_{\Gamma_{0}}^{1}(\omega), & \mathcal{R}_{2} \in H^{2}(\omega) \cap H_{\Gamma_{0}}^{1}(\omega), & \\
\mathcal{U}_{1} \in H^{2}(\omega) \cap H_{\Gamma_{0}}^{1}(\omega), & \mathcal{U}_{2} \in H^{4}(\omega) \cap H_{\Gamma_{0}}^{1}(\omega), \quad \mathcal{U}_{3} \in H^{2}(\omega) \cap H_{\Gamma_{0}}^{1}(\omega) .
\end{array}
$$

\section{UNFOLDING OF THE RESCALED SHELL}

Definition 4.1. The unfolding $\mathcal{T}_{\varepsilon}\left(\psi^{\prime}\right)$ (resp. $\mathcal{T}_{\varepsilon}(\psi)$ ) of a measurable function defined on $\omega$ (resp. $\Omega$ ) is measurable on $\omega \times Y^{\prime}$ (resp. $\Omega \times Y^{\prime}$ ) and given by

$$
\begin{array}{llll}
\mathcal{T}_{\varepsilon}\left(\psi^{\prime}\right)\left(s^{\prime}, y^{\prime}\right)=\psi^{\prime}\left(\varepsilon\left[\frac{s^{\prime}}{\varepsilon}\right]_{Y^{\prime}}+\varepsilon y^{\prime}\right) & \text { for } & \text { a.e. } & \left(s^{\prime}, y^{\prime}\right) \in \widehat{\omega}_{\varepsilon} \times Y^{\prime}, \\
\mathcal{T}_{\varepsilon}(\psi)\left(s^{\prime}, y^{\prime}\right)=0 & \text { for } & \text { a.e. } & \left(s^{\prime}, y^{\prime}\right) \in \Lambda_{\varepsilon} \times Y^{\prime},
\end{array}
$$

and

$$
\begin{array}{lll}
\mathcal{T}_{\varepsilon}(\psi)\left(s^{\prime}, y^{\prime}, y_{3}\right)=\psi\left(\varepsilon\left[\frac{s^{\prime}}{\varepsilon}\right]_{Y^{\prime}}+\varepsilon y^{\prime}, y_{3}\right) & \text { for } \quad \text { a.e. } & \left(s^{\prime}, y^{\prime}, y_{3}\right) \in \widehat{\omega}_{\varepsilon} \times Y, \\
\mathcal{T}_{\varepsilon}(\psi)\left(s^{\prime}, y^{\prime}, y_{3}\right)=0 & \text { for } \quad \text { a.e. } & \left(s^{\prime}, y^{\prime}, y_{3}\right) \in \Lambda_{\varepsilon} \times Y .
\end{array}
$$

As shown in [9], for every $\psi^{\prime} \in L^{2}(\omega)$ we have

$$
\left\|\mathcal{T}_{\varepsilon}\left(\psi^{\prime}\right)\right\|_{L^{2}\left(\omega \times Y^{\prime}\right)} \leq\left\|\psi^{\prime}\right\|_{L^{2}(\omega)} .
$$

Definition 4.2. The rescaling-unfolding operator is defined by $\Pi_{\varepsilon}=\mathcal{T}_{\varepsilon} \circ \mathfrak{T}_{\varepsilon}$.

Lemma 4.3. We obtain the following estimate for the warping:

$$
\left\|\Pi_{\varepsilon}(\bar{u})\right\|_{L^{2}\left(\omega ; H^{1}(Y)\right)} \leq C \varepsilon^{1 / 2}\|e(u)\|_{L^{2}\left(\mathcal{Q}_{\varepsilon}^{*}\right)} .
$$

Denote $H_{\text {per }}^{1}\left(Y^{\prime}\right)\left(\right.$ resp. $\left.H_{\text {per }}^{1}(Y)\right)$ the subspace of $H_{\text {loc }}^{1}\left(\mathbb{R}^{2}\right)\left(\right.$ resp. $\left.H_{\text {loc }}^{1}\left(\mathbb{R}^{2} \times(-\kappa, \kappa)\right) \cap H^{1}(Y)\right)$ containing the functions $\mathbf{G}$ periodic and

$$
\widehat{\overline{\mathcal{W}}} \doteq\left\{\widehat{\bar{\pi}} \in H_{\mathrm{per}}^{1}(Y)^{3} \mid \int_{-\kappa}^{\kappa} \widehat{\bar{\pi}}\left(\cdot, y_{3}\right) \mathrm{d} y_{3}=0, \quad \int_{-\kappa}^{\kappa} y_{3} \widehat{\bar{\pi}}_{\alpha}\left(\cdot, y_{3}\right) \mathrm{d} y_{3}=0 \quad \text { a.e. } \quad \text { in } \quad \omega \times Y^{\prime}\right\} .
$$

Lemma 4.4. There exists a subsequence of $\{\varepsilon\} \quad$ (still denoted $\{\varepsilon\}$ ) and $\widehat{\mathcal{U}} \in L^{2}\left(\omega ; H_{\text {per }}^{1}\left(Y^{\prime}\right)\right)^{3}$, $\widehat{\mathcal{R}} \in L^{2}\left(\omega ; H_{\mathrm{per}}^{1}\left(Y^{\prime}\right)\right)^{2}$ and $\widehat{\bar{u}} \in L^{2}(\omega ; \widehat{\overline{\mathcal{W}}})$ such that

$$
\begin{aligned}
& \mathcal{T}_{\varepsilon}\left(\mathcal{U}_{\varepsilon}\right) \longrightarrow \mathcal{U} \text { strongly in } L^{2}\left(\omega ; H^{1}\left(Y^{\prime}\right)\right)^{3}, \\
& \mathcal{T}_{\varepsilon}\left(\mathcal{R}_{\varepsilon}\right) \longrightarrow \mathcal{R} \text { strongly in } L^{2}\left(\omega ; H^{1}\left(Y^{\prime}\right)\right)^{2}, \\
& \mathcal{T}_{\varepsilon}\left(\frac{\partial \mathcal{U}_{\varepsilon}}{\partial s_{\alpha}}\right) \longrightarrow \frac{\partial \mathcal{U}}{\partial s_{\alpha}} \text { strongly in } L^{2}\left(\omega \times Y^{\prime}\right)^{3}, \\
& \mathcal{T}_{\varepsilon}\left(\frac{\partial \mathcal{R}_{\varepsilon}}{\partial s_{\alpha}}\right) \rightarrow \frac{\partial \mathcal{R}}{\partial s_{\alpha}}+\frac{\partial \widehat{\mathcal{R}}}{\partial y_{\alpha}} \quad \text { weakly in } L^{2}\left(\omega \times Y^{\prime}\right)^{2}, \\
& \frac{1}{\varepsilon^{2}} \Pi_{\varepsilon}\left(\bar{u}_{\varepsilon}\right) \rightarrow \widehat{\bar{u}} \quad \text { weakly } \text { in } \quad L^{2}\left(\omega ; H^{1}(Y)\right)^{3} .
\end{aligned}
$$


One has

$$
\bar{u}\left(s_{1}, s_{2}, y_{3}\right)=\frac{1}{\left|Y^{\prime}\right|} \int_{Y^{\prime}} \widehat{\bar{u}}\left(s_{1}, s_{2}, y_{1}, y_{2}, y_{3}\right) \mathrm{d} y_{1} \mathrm{~d} y_{2} \quad \text { for } \quad \text { a.e. } \quad\left(s_{1}, s_{2}, y_{3}\right) \in \Omega
$$

Moreover,

$$
\begin{gathered}
\frac{1}{\varepsilon} \mathcal{T}_{\varepsilon}\left(\frac{\partial \mathcal{U}_{\varepsilon}}{\partial s_{\alpha}} \cdot \boldsymbol{n}+\mathcal{R}_{\varepsilon} \cdot \boldsymbol{t}_{\alpha}\right) \rightarrow \mathcal{Z}_{\alpha 3}+\widehat{Z}_{\alpha 3} \quad \text { weakly } \quad \text { in } \quad L^{2}\left(\omega ; H^{1}\left(Y^{\prime}\right)\right), \\
\frac{1}{\varepsilon} \mathcal{T}_{\varepsilon}\left(\frac{\partial \mathcal{U}_{\varepsilon}}{\partial s_{\alpha}} \cdot \boldsymbol{t}_{\beta}+\frac{\partial \mathcal{U}_{\varepsilon}}{\partial s_{\beta}} \cdot \boldsymbol{t}_{\alpha}\right) \rightarrow \mathcal{Z}_{\alpha \beta}+\widehat{Z}_{\alpha \beta} \quad \text { weakly } \quad \text { in } \quad L^{2}\left(\omega ; H^{1}\left(Y^{\prime}\right)\right),
\end{gathered}
$$

where

$$
\widehat{Z}_{13}=\frac{\partial \widehat{\mathcal{U}}_{3}}{\partial y_{1}}+\widehat{\mathcal{R}}_{1}, \quad \widehat{Z}_{23}=\frac{\partial \widehat{\mathcal{U}}_{3}}{\partial y_{2}}+\widehat{\mathcal{R}}_{2}, \quad \widehat{Z}_{\alpha \beta}=e_{y, \alpha \beta}(\widehat{\mathcal{U}})
$$

Proof. The strong convergences of $(4.3)_{1,2,3}$ follow from $(3.2)_{1,2}$ and Proposition 3.4 of [9]. Convergence (4.3) 4 is the consequence of Theorem 3.5 from [9] and $(4.3)_{5}$ is obtained with Corollary 3.2 of [9].

The convergences of $(4.4)_{1,2}$ follow from Lemma 3.1 and Theorem 3.5 of [9]. With Lemma A.2 we then obtain the expression for $\widehat{Z}_{\alpha 3}$ in (4.5) and Lemma A.3 yields the expression $\widehat{Z}_{\alpha \beta}$.

To do that, first we need to identify the different fields appearing in Lemma A.2. Here

$$
u_{\varepsilon} \longleftrightarrow \mathcal{U}_{\varepsilon, 3}, \quad v_{\varepsilon} \longleftrightarrow\left(\begin{array}{c}
-\frac{1}{a} \mathcal{U}_{\varepsilon, 1}+\mathcal{R}_{\varepsilon, 1} \\
\mathcal{R}_{\varepsilon, 2}
\end{array}\right)
$$

From $(3.2)_{1,4}$, one has

$$
\begin{aligned}
& \frac{1}{\varepsilon}\left(\nabla \mathcal{U}_{\varepsilon, 3}+\left(\begin{array}{c}
-\frac{1}{a} \mathcal{U}_{\varepsilon, 1}+\mathcal{R}_{\varepsilon, 1} \\
\mathcal{R}_{\varepsilon, 2}
\end{array}\right)\right) \rightarrow\left(\begin{array}{l}
\mathcal{Z}_{13} \\
\mathcal{Z}_{23}
\end{array}\right) \quad \text { weakly } \quad \text { in } \quad L^{2}(\omega)^{2} \\
& \mathcal{T}_{\varepsilon}\left[\nabla\left(\begin{array}{c}
-\frac{1}{a} \mathcal{U}_{\varepsilon, 1}+\mathcal{R}_{\varepsilon, 1} \\
\mathcal{R}_{\varepsilon, 2}
\end{array}\right)\right] \rightarrow \nabla\left(\begin{array}{c}
-\frac{1}{a} \mathcal{U}_{1}+\mathcal{R}_{1} \\
\mathcal{R}_{2}
\end{array}\right)+\nabla_{y}\left(\begin{array}{l}
\widehat{\mathcal{R}}_{1} \\
\widehat{\mathcal{R}}_{2}
\end{array}\right) \text { weakly in } \quad L^{2}\left(\omega \times Y^{\prime}\right)^{2} .
\end{aligned}
$$

Then, one can apply Lemma A.2. The function $\mathfrak{u}$ is called $\widehat{\mathcal{U}}_{3}$.

Now we determine the $\widehat{Z}_{\alpha \beta}$ 's.

Let us identify

$$
u_{\varepsilon} \longleftrightarrow\left(\begin{array}{l}
\mathcal{U}_{\varepsilon, 1} \\
\mathcal{U}_{\varepsilon, 2}
\end{array}\right), \quad v_{\varepsilon} \longleftrightarrow\left(\begin{array}{cc}
\frac{1}{a} \mathcal{U}_{\varepsilon, 3} & 0 \\
0 & 0
\end{array}\right)
$$

Hence, by $(3.2)_{1,3} \frac{1}{\varepsilon}\left(e\left(u_{\varepsilon}\right)+v_{\varepsilon}\right) \rightarrow \mathcal{X}$, and $\mathcal{T}_{\varepsilon}\left(\nabla v_{\varepsilon}\right) \rightarrow \nabla v+\nabla_{y} \widehat{v}$. Here, observe that $\widehat{v}=0$. The field $\left(\mathfrak{u}_{1}, \mathfrak{u}_{2}\right)$ given by Lemma A.3 is denoted $\left(\widehat{\mathcal{U}}_{1}, \widehat{\mathcal{U}}_{2}\right)$. 


\subsection{Limit of the rescaled-unfolded strain tensor}

Proposition 4.5. Under the assumptions and the results of Lemma 4.4 we obtain the following weak convergences in $L^{2}(\omega \times Y)$ :

$$
\begin{aligned}
\frac{1}{\varepsilon} \Pi_{\varepsilon}\left(e_{\alpha \alpha}\left(u_{\varepsilon}\right)\right) \rightarrow \mathcal{Z}_{\alpha \alpha}+e_{y, \alpha \alpha}(\widehat{\mathcal{U}})+y_{3}\left(\frac{\partial \mathcal{R}_{\alpha}}{\partial s_{\alpha}}+\frac{\partial \widehat{\mathcal{R}}_{\alpha}}{\partial y_{\alpha}}\right)+\frac{\partial \widehat{\bar{u}}_{\alpha}}{\partial y_{\alpha}}, \\
\frac{1}{\varepsilon} \Pi_{\varepsilon}\left(e_{12}\left(u_{\varepsilon}\right)\right) \rightarrow \frac{1}{2}\left(\mathcal{Z}_{12}+2 e_{y, 22}(\widehat{\mathcal{U}})+y_{3}\left(\frac{\partial \mathcal{R}_{1}}{\partial s_{2}}+\frac{\partial \widehat{\mathcal{R}}_{1}}{\partial y_{2}}+\frac{\partial \mathcal{R}_{2}}{\partial s_{1}}+\frac{\partial \widehat{\mathcal{R}}_{2}}{\partial y_{1}}\right)+\frac{\partial \widehat{\bar{u}}_{1}}{\partial y_{2}}+\frac{\partial \widehat{\bar{u}}_{2}}{\partial y_{1}}\right), \\
\frac{1}{\varepsilon} \Pi_{\varepsilon}\left(e_{\alpha 3}\left(u_{\varepsilon}\right)\right) \rightarrow \frac{1}{2}\left(\mathcal{Z}_{\alpha 3}+\frac{\partial \widehat{\mathcal{U}}_{3}}{\partial y_{\alpha}}+\widehat{\mathcal{R}}_{\alpha}+\frac{\partial \widehat{\bar{u}}_{\alpha}}{\partial y_{3}}+\frac{\partial \widehat{\bar{u}}_{3}}{\partial y_{\alpha}}\right), \\
\frac{1}{\varepsilon} \Pi_{\varepsilon}\left(e_{33}\left(u_{\varepsilon}\right)\right) \rightarrow \frac{\partial \widehat{\bar{u}}_{3}}{\partial y_{3}} .
\end{aligned}
$$

Proof. First, note that the function $y_{3} \longrightarrow \frac{a}{a+\varepsilon y_{3}}$ converges uniformly to 1 in $\omega \times Y$.

Below, we give the limits for $\frac{1}{\varepsilon} \Pi_{\varepsilon}\left(e_{11}\left(u_{\varepsilon}\right)\right)$ and $\frac{1}{\varepsilon} \Pi_{\varepsilon}\left(e_{13}\left(u_{\varepsilon}\right)\right)$, since other cases follow in a similar way. For the calculation we combine the results obtained in Lemmas 3.1 and 4.4. We have,

$$
\frac{1}{\varepsilon} \Pi_{\varepsilon}\left(e_{11}\left(u_{\varepsilon}\right)\right)=\frac{1}{\varepsilon}\left[\frac{a}{a+\varepsilon y_{3}}\left(\mathcal{T}_{\varepsilon}\left(\frac{\partial \mathcal{U}_{\varepsilon, 1}}{\partial s_{1}}+\frac{1}{a} \mathcal{U}_{\varepsilon, 3}\right)+\varepsilon y_{3} \mathcal{T}_{\varepsilon}\left(\frac{\partial \mathcal{R}_{\varepsilon, 1}}{\partial s_{1}}\right)+\Pi_{\varepsilon}\left(\frac{\partial \bar{u}_{\varepsilon, 1}}{\partial s_{1}}\right)+\frac{1}{a} \Pi_{\varepsilon}\left(\bar{u}_{\varepsilon, 3}\right)\right)\right] .
$$

Therefore, we get for each term in the limit

$$
\begin{aligned}
& \frac{1}{\varepsilon} \mathcal{T}_{\varepsilon}\left(\frac{\partial \mathcal{U}_{\varepsilon, 1}}{\partial s_{1}}+\frac{1}{a} \mathcal{U}_{\varepsilon, 3}\right) \rightarrow \mathcal{Z}_{11}+e_{y, 11}(\widehat{\mathcal{U}}) \text { weakly in } L^{2}\left(\omega \times Y^{\prime}\right), \\
& \mathcal{T}_{\varepsilon}\left(\frac{\partial \mathcal{R}_{\varepsilon, 1}}{\partial s_{1}}\right)-\frac{\partial \mathcal{R}_{1}}{\partial s_{1}}+\frac{\partial \widehat{\mathcal{R}}_{1}}{\partial y_{1}} \quad \text { weakly in } L^{2}\left(\omega \times Y^{\prime}\right), \\
& \frac{1}{\varepsilon} \Pi_{\varepsilon}\left(\frac{\partial \bar{u}_{\varepsilon, 1}}{\partial s_{1}}\right)=\frac{1}{\varepsilon^{2}} \frac{\partial \prod_{\varepsilon}\left(\bar{u}_{\varepsilon, 1}\right)}{\partial y_{1}} \rightarrow \frac{\partial \widehat{\bar{u}}_{1}}{\partial y_{1}} \quad \text { weakly in } L^{2}(\omega \times Y), \\
& \frac{1}{\varepsilon} \Pi_{\varepsilon}\left(\bar{u}_{\varepsilon, 3}\right) \rightarrow 0 \quad \text { weakly in } L^{2}(\omega \times Y) .
\end{aligned}
$$

Hence,

$$
\frac{1}{\varepsilon} \Pi_{\varepsilon}\left(e_{11}\left(u_{\varepsilon}\right)\right) \rightarrow \mathcal{Z}_{11}+e_{y, 11}(\widehat{\mathcal{U}})+y_{3}\left(\frac{\partial \mathcal{R}_{1}}{\partial s_{1}}+\frac{\partial \widehat{\mathcal{R}}_{1}}{\partial y_{1}}\right)+\frac{\partial \widehat{\bar{u}}_{1}}{\partial y_{1}} \quad \text { weakly } \quad \text { in } \quad L^{2}(\omega \times Y) .
$$

Now we focus on

$$
\begin{aligned}
\frac{1}{\varepsilon} \Pi_{\varepsilon}\left(e_{13}\left(u_{\varepsilon}\right)\right)= & \frac{1}{2} \frac{1}{\varepsilon} \frac{a}{a+\varepsilon y_{3}}\left[\mathcal{T}_{\varepsilon}\left(\frac{\partial \mathcal{U}_{\varepsilon, 3}}{\partial s_{1}}-\frac{1}{a} \mathcal{U}_{\varepsilon, 1}+\mathcal{R}_{\varepsilon, 1}\right)\right. \\
& \left.-\frac{1}{a} \Pi_{\varepsilon}\left(\bar{u}_{\varepsilon, 1}\right)+\Pi_{\varepsilon}\left(\frac{\partial \bar{u}_{\varepsilon, 3}}{\partial s_{1}}\right)+\left(1+\frac{\varepsilon y_{3}}{a}\right) \Pi_{\varepsilon}\left(\frac{\partial \bar{u}_{\varepsilon, 1}}{\partial s_{3}}\right)\right] .
\end{aligned}
$$


Similar to the previous case we calculate the limits of each component, obtaining

$$
\begin{aligned}
& \frac{1}{\varepsilon} \mathcal{T}_{\varepsilon}\left(\frac{\partial \mathcal{U}_{\varepsilon, 3}}{\partial s_{1}}-\frac{1}{a} \mathcal{U}_{\varepsilon, 1}+\mathcal{R}_{\varepsilon, 1}\right) \rightarrow \mathcal{Z}_{13}+\frac{\partial \widehat{\mathcal{U}}_{3}}{\partial y_{1}}+\widehat{\mathcal{R}}_{1} \quad \text { weakly in } L^{2}\left(\omega \times Y^{\prime}\right), \\
& \frac{1}{\varepsilon} \Pi_{\varepsilon}\left(\bar{u}_{\varepsilon, 1}\right) \rightarrow 0 \quad \text { weakly in } L^{2}(\omega \times Y), \\
& \frac{1}{\varepsilon} \Pi_{\varepsilon}\left(\frac{\partial \bar{u}_{\varepsilon, 3}}{\partial s_{1}}\right)=\frac{1}{\varepsilon^{2}} \frac{\partial \Pi_{\varepsilon}\left(\bar{u}_{3}\right)}{\partial y_{1}} \rightarrow \frac{\partial \widehat{\bar{u}}_{3}}{\partial y_{1}} \quad \text { weakly in } L^{2}(\omega \times Y), \\
& \frac{1}{\varepsilon^{2}} \frac{\partial \Pi_{\varepsilon}\left(\bar{u}_{\varepsilon, 1}\right)}{\partial y_{3}} \rightarrow \frac{\partial \overline{\bar{u}}_{1}}{\partial y_{3}} \quad \text { weakly in } L^{2}(\omega \times Y) .
\end{aligned}
$$

Hence,

$$
\frac{1}{\varepsilon} \Pi_{\varepsilon}\left(e_{13}\left(u_{\varepsilon}\right)\right) \rightarrow \frac{1}{2}\left(\mathcal{Z}_{13}+\frac{\partial \widehat{\mathcal{U}}_{3}}{\partial y_{1}}+\widehat{\mathcal{R}}_{1}+\frac{\partial \widehat{\bar{u}}_{3}}{\partial y_{1}}+\frac{\partial \widehat{\bar{u}}_{1}}{\partial y_{3}}\right)
$$

Define the displacement $\widehat{u}$ belonging to $L^{2}\left(\omega ; H_{\text {per }}^{1}(Y)\right)^{3}$ by

$$
\widehat{u}(\cdot, y)=\widehat{\mathcal{U}}\left(\cdot, y_{1}, y_{2}\right)+y_{3} \widehat{\mathcal{R}}\left(\cdot, y_{1}, y_{2}\right)+\left(y_{3}\left(\mathcal{Z}_{13} \mathbf{t}_{1}+\mathcal{Z}_{23} \mathbf{t}_{2}\right)+\widehat{\bar{u}}(\cdot, y)\right), \quad \text { for a.e. } y \in Y^{*} \text { and a.e. in } \omega \text {. }
$$

Hence, one obtains

$$
\begin{aligned}
& \frac{1}{\varepsilon} \Pi_{\varepsilon}\left(e\left(u_{\varepsilon}\right)\right) \rightarrow\left(\begin{array}{ccc}
\mathcal{Z}_{11}+\frac{y_{3}}{a} \frac{\partial \mathcal{U}_{1}}{\partial s_{1}}-y_{3} \frac{\partial^{2} \mathcal{U}_{3}}{\partial s_{1}^{2}} & \frac{1}{2} \mathcal{Z}_{12}+\frac{y_{3}}{a} \frac{\partial \mathcal{U}_{1}}{\partial s_{2}}-y_{3} \frac{\partial^{2} \mathcal{U}_{3}}{\partial s_{1} \partial s_{2}} & 0 \\
* & \mathcal{Z}_{22}-y_{3} \frac{\partial^{2} \mathcal{U}_{3}}{\partial s_{2}^{2}} & 0 \\
* & * & 0
\end{array}\right) \\
& \quad+\mathcal{E}_{y}(\widehat{u}) \text { weakly in } L^{2}(\omega \times Y)^{3 \times 3},
\end{aligned}
$$

where $\mathcal{E}_{y}(\widehat{u})$ is the symmetric tensor whose components are the $e_{y, i j}(\widehat{u})$ 's. We want to note here that we obtain the same kind of result in [15].

The aim of the following section is to determine the $\mathcal{Z}_{\alpha \beta}$ 's.

Remark 4.6. If we compare our results with Proposition 11.13 of [14], we see that

$$
\mathcal{E}_{y}(\widehat{u})=E_{w}\left(\stackrel{u}{)}+\mathcal{E}_{y}^{1}(\widehat{u}),\right.
$$

where the terms on the right hand side follow from the given definitions in [14].

\section{InEXTENSIONAL AND EXTENSIONAL DisPLACEMENTS}

\subsection{Inextensional displacements}

Denote $\mathbb{H} \doteq\left[H_{\Gamma_{0}}^{1}(\omega)\right]^{2} \times L^{2}(\omega)$. We equip $\mathbb{H}$ with the scalar product

$$
\begin{aligned}
\langle\mathcal{U}, \mathcal{V}\rangle= & \int_{\omega}\left[\frac{1}{2}\left(\frac{\partial \mathcal{U}_{1}}{\partial s_{1}}+\frac{1}{a} \mathcal{U}_{3}\right)\left(\frac{\partial \mathcal{V}_{1}}{\partial s_{1}}+\frac{1}{a} \mathcal{V}_{3}\right)+\frac{\partial \mathcal{U}_{2}}{\partial s_{2}} \frac{\partial \mathcal{V}_{2}}{\partial s_{2}}\right. \\
& \left.+\frac{1}{2}\left(\frac{\partial \mathcal{U}_{1}}{\partial s_{2}}+\frac{\partial \mathcal{U}_{2}}{\partial s_{1}}\right)\left(\frac{\partial \mathcal{V}_{1}}{\partial s_{2}}+\frac{\partial \mathcal{V}_{2}}{\partial s_{1}}\right)+\mathcal{U}_{3} \mathcal{V}_{3}\right] \mathrm{d} s_{1} \mathrm{~d} s_{2}
\end{aligned}
$$


The associated norm is equivalent to the usual norm of $\left[H_{\Gamma_{0}}^{1}(\omega)\right]^{2} \times L^{2}(\omega)$.

Denote $D_{I}$ the space of inextensional displacements

$$
D_{I} \doteq\left\{\Phi \in \mathbb{H} \quad \mid \quad \frac{\partial \Phi_{1}}{\partial s_{1}}+\frac{1}{a} \Phi_{3}=0, \quad \frac{\partial \Phi_{2}}{\partial s_{2}}=0, \quad \frac{\partial \Phi_{1}}{\partial s_{2}}+\frac{\partial \Phi_{2}}{\partial s_{1}}=0\right\} .
$$

A displacement $\mathcal{V}$ belongs to $D_{I}$ if and only if there exists $\left(V_{1}, V_{2}\right) \in H_{0}^{1}(0, a \pi) \times H_{0}^{2}(0, a \pi)$ such that for a.e. $\left(s_{1}, s_{2}\right) \in \omega$

$$
\begin{aligned}
& \mathcal{V}_{2}\left(s_{1}, s_{2}\right)=V_{2}\left(s_{1}\right), \\
& \mathcal{V}_{1}\left(s_{1}, s_{2}\right)=-s_{2}^{c} V_{2}^{\prime}\left(s_{1}\right)+V_{1}\left(s_{1}\right), \quad V_{1} \in H_{0}^{1}(0, a \pi), \quad V_{2} \in H_{0}^{2}(0, a \pi), \\
& \mathcal{V}_{3}\left(s_{1}, s_{2}\right)=a\left(s_{2}^{c} V_{2}^{\prime \prime}\left(s_{1}\right)-V_{1}^{\prime}\left(s_{1}\right)\right) .
\end{aligned}
$$

Here we set

$$
s_{1}^{c}=s_{1}-\frac{a \pi}{2}, \quad s_{2}^{c}=s_{2}-\frac{l}{2} .
$$

The map $\mathcal{V} \in D_{I} \longmapsto\left(V_{1}, V_{2}\right) \in H_{0}^{1}(0, a \pi) \times H_{0}^{2}(0, a \pi)$ is one to one and onto.

Denote

$$
\mathbb{D}_{I}=D_{I} \cap\left(\left[H_{\Gamma_{0}}^{1}(\omega)\right]^{2} \times H_{\Gamma_{0}}^{2}(\omega)\right) .
$$

Note that the limit of the mid surface displacement of the shell $\mathcal{U}$ belongs to $\mathbb{D}_{I}$.

We equip $D_{I}\left(\right.$ resp. $\left.\mathbb{D}_{I}\right)$ with the semi-norm

$$
\|\mathcal{V}\|_{D_{I}}=\left\|\mathcal{V}_{3}\right\|_{L^{2}(\omega)}, \quad\left(\text { resp. }\|\mathcal{V}\|_{\mathbb{D}_{I}}=\left\|\mathcal{V}_{3}\right\|_{H^{2}(\omega)}\right) .
$$

Lemma 5.1. The semi-norm $\|\cdot\|_{D_{I}}$ (resp. $\|\cdot\|_{\mathbb{D}_{I}}$ ) is a norm equivalent to the norm of the product space $\left[H^{1}(\omega)\right]^{2} \times L^{2}(\omega)$ (resp. $\left.\left[H^{1}(\omega)\right]^{2} \times H^{2}(\omega)\right)$.

Moreover, there exist two constants $c, C$ such that for every $\mathcal{V} \in D_{I}$ (resp. $\mathcal{V} \in \mathbb{D}_{I}$ ) one has

$$
\begin{aligned}
c\left(\left\|V_{1}\right\|_{H_{0}^{1}(0, a \pi)}^{2}+\left\|V_{2}\right\|_{H_{0}^{2}(0, a \pi)}^{2}\right) & \leq\|\mathcal{V}\|_{D_{I}}^{2} \leq C\left(\left\|V_{1}\right\|_{H_{0}^{1}(0, a \pi)}^{2}+\left\|V_{2}\right\|_{H_{0}^{2}(0, a \pi)}^{2}\right), \\
\text { (resp. } c\left(\left\|V_{1}\right\|_{H_{0}^{3}(0, a \pi)}^{2}+\left\|V_{2}\right\|_{H_{0}^{4}(0, a \pi)}^{2}\right) & \left.\leq\|\mathcal{V}\|_{\mathbb{D}_{I}}^{2} \leq C\left(\left\|V_{1}\right\|_{H_{0}^{3}(0, a \pi)}^{2}+\left\|V_{2}\right\|_{H_{0}^{4}(0, a \pi)}^{2}\right)\right)
\end{aligned}
$$

where $\left(V_{1}, V_{2}\right)$ are associated to $\mathcal{V}$ by $(5.1)$.

Proof. see Appendix A.

\subsection{Extensional displacements}

Denote $D_{E}$ the orthogonal subspace of $D_{I}$ in $\mathbb{H}$ for the scalar product of $\mathbb{H}$.

For every $\phi$ in $L^{2}(\omega)$, denote

$$
\mathcal{M}_{2}(\phi)\left(s_{1}\right)=\frac{1}{l} \int_{0}^{l} \phi\left(s_{1}, s_{2}\right) \mathrm{d} s_{2}, \quad \mathcal{M}_{2}^{c}(\phi)\left(s_{1}\right)=\frac{1}{l} \int_{0}^{l} \phi\left(s_{1}, s_{2}\right) s_{2}^{c} \mathrm{~d} s_{2} \quad \text { for } \quad \text { a.e. } \quad s_{1} \in(0, a \pi) .
$$

Note that for every $\mathcal{U} \in D_{E}$, one has $\mathcal{M}_{2}\left(\mathcal{U}_{\alpha}\right), \mathcal{M}_{2}^{c}\left(\mathcal{U}_{\alpha}\right) \in H_{0}^{1}(0, a \pi)$ while $\mathcal{M}_{2}\left(\mathcal{U}_{3}\right), \mathcal{M}_{2}^{c}\left(\mathcal{U}_{3}\right) \in L^{2}(0, a \pi)$ $(\alpha \in\{1,2\})$.

Let $\mathcal{U}$ be in $D_{E}$, it satisfies $\langle\mathcal{U}, \mathcal{V}\rangle=\int_{\omega} \mathcal{U}_{3} \mathcal{V}_{3} \mathrm{~d} s_{1} \mathrm{~d} s_{2}, \quad \forall \mathcal{V} \in D_{I} . \quad$ Thus,

$$
\int_{\omega} \mathcal{U}_{3}\left(s_{1}, s_{2}\right)\left(s_{2}^{c} V_{2}^{\prime \prime}\left(s_{1}\right)-V_{1}^{\prime}\left(s_{1}\right)\right) \mathrm{d} s_{1} \mathrm{~d} s_{2}=0, \quad \forall V_{1} \in H_{0}^{1}(0, a \pi), \quad \forall V_{2} \in H_{0}^{2}(0, a \pi) .
$$

That gives

$$
\begin{aligned}
& \mathcal{M}_{2}\left(\mathcal{U}_{3}\right)\left(s_{1}\right)=C_{1}, \quad \mathcal{M}_{2}^{c}\left(\mathcal{U}_{3}\right)\left(s_{1}\right)=C_{2} s_{1}^{c}+C_{3}, \\
& \left(C_{1}, C_{2}, C_{3}\right) \in \mathbb{R}^{3} \quad \text { for } \quad \text { a.e. } \quad s_{1} \in(0, a \pi) .
\end{aligned}
$$


Hence

$$
D_{E}=\left\{\mathcal{U} \in \mathbb{H} \mid \frac{\mathrm{d} \mathcal{M}_{2}\left(\mathcal{U}_{3}\right)}{\mathrm{d} s_{1}}=\frac{\mathrm{d}^{2} \mathcal{M}_{2}^{c}\left(\mathcal{U}_{3}\right)}{\mathrm{d} s_{1}^{2}}=0 \quad \text { in } \quad(0, a \pi)\right\} .
$$

We equip $D_{E}$ with the norm

$$
\|\Phi\|_{E}=\sqrt{\int_{\omega}\left[\frac{1}{2}\left|\frac{\partial \Phi_{1}}{\partial s_{1}}+\frac{1}{a} \Phi_{3}\right|^{2}+\left|\frac{\partial \Phi_{2}}{\partial s_{2}}\right|^{2}+\frac{1}{2}\left|\frac{\partial \Phi_{1}}{\partial s_{2}}+\frac{\partial \Phi_{2}}{\partial s_{1}}\right|^{2}\right] \mathrm{d} s_{1} \mathrm{~d} s_{2} .}
$$

Endowed with this norm, $D_{E}$ is not a Hilbert space. We denote with $\mathbb{D}_{E}$ the completion of $D_{E}$ for this norm.

Lemma 5.2. For every $\mathcal{U}$ in $\mathbb{D}_{E}$, one has

$$
\left\|\mathcal{U}_{2}\right\|_{H^{1}\left(0, l ; L^{2}(0, a \pi)\right)}+\left\|\mathcal{U}_{1}\right\|_{H^{1}\left(0, l ;\left(H^{1}(0, a \pi)\right)^{\prime}\right)}+\left\|\mathcal{U}_{3}\right\|_{L^{2}\left(0, l ;\left(H^{2}(0, a \pi)\right)^{\prime}\right)} \leq C\|\mathcal{U}\|_{E}
$$

Proof. See Appendix A.

Now, consider the field $\mathcal{U}_{\varepsilon}$, the mid-surface displacement associated to $u_{\varepsilon}$ the solution of the variational problem (6.5). This field belongs to $\mathbb{H}$. We decompose it as the sum of an inextensional displacement $\mathcal{U}_{I, \varepsilon}$ and an extensional one $\mathcal{U}_{E, \varepsilon}$. By the definition of $\|\cdot\|_{E}$ and Lemma 1.7 we obtain

$$
\left\|\mathcal{U}_{E, \varepsilon}\right\|_{E} \leq \sum_{\alpha, \beta=1}^{2}\left\|\frac{\partial \mathcal{U}_{\varepsilon}}{\partial s_{\alpha}} \cdot \mathbf{t}_{\beta}+\frac{\partial \mathcal{U}_{\varepsilon}}{\partial s_{\beta}} \cdot \mathbf{t}_{\alpha}\right\|_{L^{2}(\omega)} \leq \frac{C}{\varepsilon^{1 / 2}}\left\|e\left(u_{\varepsilon}\right)\right\|_{L^{2}\left(\mathcal{Q}_{\varepsilon}^{*}\right)} \leq C \varepsilon .
$$

Lemma 5.3. There exist a subsequence (still denoted $\{\varepsilon\}$ ) and $\mathcal{U}_{E} \in \mathbb{D}_{E}$ such that

$$
\begin{aligned}
& \frac{1}{\varepsilon} \mathcal{U}_{E, \varepsilon, 1} \rightarrow \mathcal{U}_{E, 1} \quad \text { weakly } \quad \text { in } \quad H^{1}\left(0, l ;\left(H^{1}(0, a \pi)\right)^{\prime}\right), \\
& \frac{1}{\varepsilon} \mathcal{U}_{E, \varepsilon, 2} \rightarrow \mathcal{U}_{E, 2} \quad \text { weakly } \quad \text { in } \quad H^{1}\left(0, l ; L^{2}(0, a \pi)\right), \\
& \frac{1}{\varepsilon} \mathcal{U}_{E, \varepsilon, 3} \rightarrow \mathcal{U}_{E, 3} \quad \text { weakly } \quad \text { in } \quad L^{2}\left(0, l ;\left(H^{2}(0, a \pi)\right)^{\prime}\right) .
\end{aligned}
$$

Proof. From Lemma 5.2, one has

$$
\left\|\mathcal{U}_{E, \varepsilon, 1}\right\|_{H^{1}\left(0, l ;\left(H^{1}(0, a \pi)\right)^{\prime}\right)}+\left\|\mathcal{U}_{E, \varepsilon, 2}\right\|_{H^{1}\left(0, l ; L^{2}(0, a \pi)\right)}+\left\|\mathcal{U}_{E, \varepsilon, 3}\right\|_{L^{2}\left(0, l ;\left(H^{2}(0, a \pi)\right)^{\prime}\right)} \leq C \varepsilon
$$

which yields the claim.

Going back to the expressions for $\mathcal{Z}_{\alpha \beta}$ introduced Lemma 3.1 and Proposition 4.5 we get with Lemma 5.3 that

$$
\mathcal{Z}_{\alpha \beta}=\frac{1}{2}\left[\frac{\partial \mathcal{U}_{E}}{\partial s_{\alpha}} \mathbf{t}_{\beta}+\frac{\partial \mathcal{U}_{E}}{\partial s_{\beta}} \mathbf{t}_{\alpha}\right]
$$

\section{THE LINEAR ELASTICITY PROBLEM}

Let $a_{i j k l} \in L^{\infty}(Y), i, j, k, l \in\{1,2,3\}$ and it should satisfy both the symmetry condition

$$
a_{i j k l}(y)=a_{j i k l}(y)=a_{k l i j}(y) \text { for } \text { a.e. } y \in Y,
$$

and the coercivity condition $\left(c_{0}>0\right)$

$$
a_{i j k l}(y) \tau_{i j} \tau_{k l} \geq c_{0} \tau_{i j} \tau_{i j} \quad \text { for } \quad \text { a.e. } \quad y \in Y,
$$


where $\tau$ is a $3 \times 3$ symmetric real matrix.

The coefficients $a_{i j k l}^{\varepsilon}$ of the Hooke's tensor on the shell for $x=\Phi(s)$ are given by

$$
\begin{aligned}
a_{i j k l}^{\varepsilon}(x) & =a_{i j k l}\left(\left\{\frac{s^{\prime}}{\varepsilon}\right\}_{Y^{\prime}}, \frac{s_{3}}{\varepsilon}\right) \quad \text { for } \quad \text { a.e. } x \in \mathcal{Q}_{\varepsilon}^{*}, \\
\sigma_{i j}^{\varepsilon}(v) & =a_{i j k l}^{\varepsilon} e_{k l}(v) \quad \forall v \in V_{\varepsilon}^{*} .
\end{aligned}
$$

For a given applied force $f_{\varepsilon}$ the displacement $u_{\varepsilon}$ of a shell is the solution to the linear elasticity problem

$$
\left\{\begin{array}{l}
\text { Find } u_{\varepsilon} \in V_{\varepsilon}^{*} \text { such that } \\
\int_{\mathcal{Q}_{\varepsilon}^{*}} \sigma^{\varepsilon}\left(u_{\varepsilon}\right): e(v) \mathrm{d} x=\int_{\mathcal{Q}_{\varepsilon}^{*}} f_{\varepsilon} v \mathrm{~d} x, \quad \forall v \in V_{\varepsilon}^{*},
\end{array}\right.
$$

where the colon denotes the classical Frobenius scalar product.

\subsection{Assumptions on the forces}

We assume that the body forces are given by

$$
f_{\varepsilon}\left(s_{1}, s_{2}, s_{3}\right)=\varepsilon^{2} f\left(s_{1}, s_{2}\right)+\varepsilon F\left(s_{1}, s_{2}\right)+s_{3} g\left(s_{1}, s_{2}\right) \quad \text { for } \quad \text { a.e. } \quad\left(s_{1}, s_{2}\right) \in \omega,
$$

where $f=f_{1} \mathbf{t}_{1}+f_{2} \mathbf{t}_{2}+f_{3} \mathbf{n},\left(f_{1}, f_{2}, f_{3}\right) \in L^{2}(\omega)^{3}$ and $g=g_{1} \mathbf{t}_{1}+g_{2} \mathbf{t}_{2},\left(g_{1}, g_{2}\right) \in L^{2}(\omega)^{2}$.

Regarding $F$, we want to choose this field so that it does not act with inextensional displacements. First, in view of Lemma 5.2 , we take

$$
F_{1} \in L^{2}\left(0, l ; H^{1}(0, a \pi)\right), \quad F_{2} \in L^{2}(\omega), \quad F_{3} \in L^{2}\left(0, l ; H^{2}(0, a \pi)\right) .
$$

Then

$$
\int_{\omega} F\left(s_{1}, s_{2}\right) \cdot V\left(s_{1}, s_{2}\right) \mathrm{d} s_{1} \mathrm{~d} s_{2}, \quad V \in D_{E},
$$

will be written

$$
\begin{aligned}
\langle F, V\rangle= & \int_{0}^{l}\left\langle F_{1}, V_{1}\right\rangle_{\left.H^{1}(0, a \pi),\left(H^{1}(0, a \pi)\right)^{\prime}\right)} \mathrm{d} s_{2}+\int_{\omega} F_{2} V_{2} \mathrm{~d} s_{1} \mathrm{~d} s_{2} \\
& +\int_{0}^{l}\left\langle F_{3}, V_{3}\right\rangle_{\left.H^{2}(0, a \pi),\left(H^{2}(0, a \pi)\right)^{\prime}\right)} \mathrm{d} s_{2},
\end{aligned}
$$

for every $V \in \mathbb{D}_{E}$. Due to Lemma 5.2, one has

$$
|\langle F, V\rangle| \leq\left(\left\|F_{1}\right\|_{L^{2}\left(0, L ; H^{1}(0, a \pi)\right)}+\left\|F_{2}\right\|_{L^{2}(\omega)}+\left\|F_{3}\right\|_{L^{2}\left(0, L ; H^{2}(0, a \pi)\right)}\right)\|V\|_{E}, \quad \forall V \in \mathbb{D}_{E} .
$$

Recall that this field has to satisfy for all $\mathcal{V} \in D_{I}$ that

$$
\int_{\omega} F\left(s_{1}, s_{2}\right) \cdot \mathcal{V}\left(s_{1}, s_{2}\right) \mathrm{d} s=0 .
$$

Hence, for all $\left(V_{1}, V_{2}\right) \in H_{0}^{1}(0, a \pi) \times H_{0}^{2}(0, a \pi)$

$$
\int_{\omega}\left(\begin{array}{c}
F_{1}\left(s_{1}, s_{2}\right) \\
F_{2}\left(s_{1}, s_{2}\right) \\
F_{3}\left(s_{1}, s_{2}\right)
\end{array}\right) \cdot\left(\begin{array}{c}
-s_{2}^{c} V_{2}^{\prime}\left(s_{1}\right)+V_{1}\left(s_{1}\right) \\
V_{2}\left(s_{1}\right) \\
a\left(s_{2}^{c} V_{2}^{\prime \prime}\left(s_{1}\right)-V_{1}^{\prime}\left(s_{1}\right)\right)
\end{array}\right) \mathrm{d} s=0 .
$$


We then get with partial integration and the boundary conditions for $V_{1}$ and $V_{2}$ that

$$
\int_{\omega}\left[\left(\frac{\partial F_{1}}{\partial s_{1}} s_{2}^{c}+F_{2}+a \frac{\partial^{2} F_{3}}{\partial s_{1}^{2}} s_{2}^{c}\right) V_{2}+\left(F_{1}+a \frac{\partial F_{3}}{\partial s_{1}}\right) V_{1}\right] \mathrm{d} s_{1} \mathrm{~d} s_{2}=0,
$$

holds for all $V_{1} \in H_{0}^{1}(0, a \pi)$ and $V_{2} \in H_{0}^{2}(0, a \pi)$. Hence, the field $F \in L^{2}(\omega)^{3}$ has to satisfy ${ }^{4}$

$$
\mathcal{M}_{2}\left(F_{1}\right)+a \frac{\mathrm{d} \mathcal{M}_{2}\left(F_{3}\right)}{\mathrm{d} s_{1}}=0, \quad \frac{\mathrm{d} \mathcal{M}_{2}^{c}\left(F_{1}\right)}{\mathrm{d} s_{1}}+\mathcal{M}_{2}\left(F_{2}\right)+a \frac{\mathrm{d}^{2} \mathcal{M}_{2}^{c}\left(F_{3}\right)}{\mathrm{d} s_{1}^{2}}=0 .
$$

In Lemma 9.3 we show that there exists a field $\mathbf{F} \in L^{2}(\omega)^{3}$ such that

$$
\langle F, V\rangle=\int_{\omega}\left(\mathbf{F}_{11} e_{11}(V)+\mathbf{F}_{12} e_{12}(V)+\mathbf{F}_{22} e_{22}(V)\right) \mathrm{d} s_{1} \mathrm{~d} s_{2} .
$$

Taking into account the holes, we need an additional assumption on the forces $F$. We will see this in the proof of the lemma below.

From now on, we assume that $F$ satisfies (6.6) and moreover $F \in H^{1}(\omega)^{3}$.

Lemma 6.1. One has

$$
\begin{gathered}
\mid \frac{1}{2 \kappa} \int_{\mathcal{Q}_{\varepsilon}^{*}} f_{\varepsilon} \cdot u \mathrm{~d} x-\varepsilon^{3}\left(\int_{\omega_{\varepsilon}^{*}} f \cdot \mathcal{U} \mathrm{d} s_{1} \mathrm{~d} s_{2}+\frac{1}{\varepsilon} \int_{\omega_{\varepsilon}^{*}} F \cdot \mathcal{U}_{E} \mathrm{~d} s_{1} \mathrm{~d} s_{2}\right. \\
\left.\quad+\frac{\kappa^{2}}{3 a} \int_{\omega_{\varepsilon}^{*}} g_{\alpha} \mathcal{U}_{\alpha} \mathrm{d} s_{1} \mathrm{~d} s_{2}+\frac{\kappa^{2}}{3} \int_{\omega_{\varepsilon}^{*}} g_{\alpha} \mathcal{R}_{\alpha} \mathrm{d} s_{1} \mathrm{~d} s_{2}\right) \mid \\
\leq C \varepsilon^{5 / 2}\left(\|f\|_{L^{2}(\omega)}+\|g\|_{L^{2}(\omega)}+\|F\|_{L^{2}(\omega)}\right)\|e(u)\|_{L^{2}\left(\mathcal{Q}_{\varepsilon}^{*}\right)} .
\end{gathered}
$$

Furthermore

$$
\left|\int_{\mathcal{Q}_{\varepsilon}^{*}} f_{\varepsilon} \cdot u \mathrm{~d} x\right| \leq C \varepsilon^{3 / 2}\left(\|f\|_{L^{2}(\omega)}+\|g\|_{L^{2}(\omega)}+\left\|F_{3}\right\|_{L^{2}\left(0, L ; H^{2}(0, a \pi)\right)}+\|F\|_{H^{1}(\omega)}\right)\|e(u)\|_{L^{2}\left(\mathcal{Q}_{\varepsilon}^{*}\right)} .
$$

The constants do not depend on $\varepsilon$.

Proof. Using the decomposition of $u$ we can write (see Rem. 1.3)

$$
\begin{aligned}
\int_{\mathcal{Q}_{\varepsilon}^{*}} f_{\varepsilon} \cdot u \mathrm{~d} x= & \int_{\Omega_{\varepsilon}^{*}} f_{\varepsilon} \cdot u \operatorname{det}\left(\mathbf{t}_{1}+\frac{s_{3}}{a} \mathbf{t}_{1}\left|\mathbf{t}_{2}\right| \mathbf{n}\right) \mathrm{d} s \\
= & \varepsilon^{3} 2 \kappa \int_{\omega_{\varepsilon}^{*}} f \cdot \mathcal{U} \mathrm{d} s_{1} \mathrm{~d} s_{2}+2 \kappa \varepsilon^{2} \int_{\omega_{\varepsilon}^{*}} F \cdot \mathcal{U}_{E} \mathrm{~d} s_{1} \mathrm{~d} s_{2}+\frac{2 \varepsilon^{3} \kappa^{3}}{3 a} \int_{\omega_{\varepsilon}^{*}} g_{\alpha} \mathcal{U}_{\alpha} \mathrm{d} s_{1} \mathrm{~d} s_{2} \\
& +\frac{2 \varepsilon^{3} \kappa^{3}}{3} \int_{\omega_{\varepsilon}^{*}} g_{\alpha} \mathcal{R}_{\alpha} \mathrm{d} s_{1} \mathrm{~d} s_{2}+\frac{2 \varepsilon^{4} \kappa^{3}}{3 a} \int_{\omega_{\varepsilon}^{*}} F \cdot \mathcal{R} \mathrm{d} s_{1} \mathrm{~d} s_{2}+\frac{2 \varepsilon^{5} \kappa^{3}}{3 a} \int_{\omega_{\varepsilon}^{*}} f_{\alpha} \mathcal{R}_{\alpha} \mathrm{d} s_{1} \mathrm{~d} s_{2} \\
& +\int_{\Omega_{\varepsilon}^{*}} \frac{s_{3}^{2}}{a} g \cdot \bar{u} d s+\int_{\Omega_{\varepsilon}^{*}} \frac{\varepsilon}{a} s_{3} F \cdot \bar{u} d s+\int_{\Omega_{\varepsilon}^{*}} \frac{s_{3}}{a} \varepsilon^{2} f_{3} \bar{u} \cdot \mathbf{n} \mathrm{d} s .
\end{aligned}
$$

${ }^{4}$ As example, take $\left(\mathcal{F}_{2}, \mathcal{F}_{3}\right) \in L^{2}(0, a \pi) \times H^{2}(0, a \pi)$ and set

$$
F\left(s_{1}, s_{2}\right)=s_{2}^{c}\left(-a \frac{\mathrm{d} \mathcal{F}_{3}}{\mathrm{~d} s_{1}}\left(s_{1}\right) \mathbf{t}_{1}+\mathcal{F}_{2}\left(s_{1}\right) \varepsilon_{2}+\mathcal{F}_{3}\left(s_{1}\right) \mathbf{n}\right) \quad \text { for } \quad \text { a.e. } \quad\left(s_{1}, s_{2}\right) \in \omega .
$$


First, using the estimates $(1.19)_{2}$ and $(1.21)_{2}$ one gets

$$
\begin{gathered}
\left|\int_{\Omega_{\varepsilon}^{*}} \varepsilon s_{3}^{2} g \cdot \bar{u} \mathrm{~d} s\right| \leq C \varepsilon^{9 / 2}\|g\|_{L^{2}(\omega)}\|e(u)\|_{L^{2}\left(\mathcal{Q}_{\varepsilon}^{*}\right)}, \quad\left|\int_{\Omega_{\varepsilon}^{*}} \varepsilon s_{3} F \cdot \bar{u} \mathrm{~d} s\right| \leq C \varepsilon^{7 / 2}\|F\|_{L^{2}(\omega)}\|e(u)\|_{L^{2}\left(\mathcal{Q}_{\varepsilon}^{*}\right)}, \\
\left|\int_{\Omega_{\varepsilon}^{*}} \varepsilon^{2} s_{3} f_{3} \bar{u} \cdot \mathbf{n} \mathrm{d} s\right| \leq C \varepsilon^{9 / 2}\|f\|_{L^{2}(\omega)}\|e(u)\|_{L^{2}\left(\mathcal{Q}_{\varepsilon}^{*}\right)}, \quad\left|\int_{\omega_{\varepsilon}^{*}} \varepsilon^{4} F \cdot \mathcal{R} \mathrm{d} s_{1} \mathrm{~d} s_{2}\right| \leq C \varepsilon^{5 / 2}\|F\|_{L^{2}(\omega)}\|e(u)\|_{L^{2}\left(\mathcal{Q}_{\varepsilon}^{*}\right)}, \\
\left|\int_{\omega_{\varepsilon}^{*}} \varepsilon^{5} f_{\alpha} \mathcal{R}_{\alpha} \mathrm{d} s_{1} \mathrm{~d} s_{2}\right| \leq C \varepsilon^{7 / 2}\|F\|_{L^{2}(\omega)}\|e(u)\|_{L^{2}\left(\mathcal{Q}_{\varepsilon}^{*}\right)} .
\end{gathered}
$$

Hence, (6.9) is proved. Now, $(1.21)_{2}$ also leads to

$$
\begin{aligned}
& \left|\varepsilon^{3} 2 \kappa \int_{\omega_{\varepsilon}^{*}} f \cdot \mathcal{U} \mathrm{d} s_{1} \mathrm{~d} s_{2}+\frac{2 \varepsilon^{3} \kappa^{3}}{3 a} \int_{\omega_{\varepsilon}^{*}} g_{\alpha} \mathcal{U}_{\alpha} \mathrm{d} s_{1} \mathrm{~d} s_{2}+\frac{2 \varepsilon^{3} \kappa^{3}}{3} \int_{\omega_{\varepsilon}^{*}} g_{\alpha} \mathcal{R}_{\alpha} \mathrm{d} s_{1} \mathrm{~d} s_{2}\right| \\
& \quad \leq C \varepsilon^{3 / 2}\left(\|f\|_{L^{2}(\omega)}+\|g\|_{L^{2}(\omega)}\right)\|e(u)\|_{L^{2}\left(\mathcal{Q}_{\varepsilon}^{*}\right)} .
\end{aligned}
$$

Now, it remains to estimate $\int_{\omega_{\varepsilon}^{*}} F \cdot \mathcal{U}_{E} \mathrm{~d} s_{1} \mathrm{~d} s_{2}$. For every function $\phi$ in $L^{1}(\omega)$, we denote

$$
\mathcal{M}_{\varepsilon}(\phi)\left(s^{\prime}\right)=\frac{1}{\varepsilon^{2}\left|Y^{\prime}\right|} \int_{Y^{\prime}} \phi\left(\varepsilon\left[\frac{s^{\prime}}{\varepsilon}\right]_{Y^{\prime}}+\varepsilon z\right) \mathrm{d} z_{1} \mathrm{~d} z_{2}, \quad \text { for } \quad \text { a.e. } \quad s^{\prime} \in \widehat{\omega}_{\varepsilon} .
$$

Function $\mathcal{M}_{\varepsilon}(\phi)$ belongs to $L^{1}\left(\widehat{\omega}_{\varepsilon}\right)$ (see $[9,10]$ for the properties of the operator $\mathcal{M}_{\varepsilon}$ ).

Recall that by (1.22), (1.21) $)_{2}$, Lemma 5.1 and (5.3) one has

$$
\|\mathcal{U}\|_{E} \leq \frac{C}{\varepsilon^{1 / 2}}\|e(u)\|_{L^{2}\left(\mathcal{Q}_{\varepsilon}^{*}\right)}, \quad\left\|\mathcal{U}_{E}\right\|_{H^{1}(\omega)} \leq \frac{C}{\varepsilon^{3 / 2}}\|e(u)\|_{L^{2}\left(\mathcal{Q}_{\varepsilon}^{*}\right)} .
$$

One has (see [10], Prop. 1.38)

$$
\begin{array}{r}
\left|\int_{\widehat{\omega}_{\varepsilon}^{*}} F \cdot \mathcal{U}_{E} \mathrm{~d} s_{1} \mathrm{~d} s_{2}-\int_{\widehat{\omega}_{\varepsilon}^{*}} F \cdot \mathcal{M}_{\varepsilon}\left(\mathcal{U}_{E}\right) \mathrm{d} s_{1} \mathrm{~d} s_{2}\right| \leq C \varepsilon\left\|\nabla \mathcal{U}_{E}\right\|_{L^{2}(\omega)}\|F\|_{L^{2}(\omega)}, \\
\left|\int_{\widehat{\omega}_{\varepsilon}^{*}} F \cdot \mathcal{M}_{\varepsilon}\left(\mathcal{U}_{E}\right) \mathrm{d} s_{1} \mathrm{~d} s_{2}-\int_{\widehat{\omega}_{\varepsilon}^{*}} \mathcal{M}_{\varepsilon}(F) \cdot \mathcal{M}_{\varepsilon}\left(\mathcal{U}_{E}\right) \mathrm{d} s_{1} \mathrm{~d} s_{2}\right| \leq C \varepsilon\left\|\mathcal{U}_{E}\right\|_{L^{2}(\omega)}\|\nabla F\|_{L^{2}(\omega)} .
\end{array}
$$

Hence

$$
\left|\int_{\widehat{\omega}_{\varepsilon}^{*}} F \cdot \mathcal{U}_{E} \mathrm{~d} s_{1} \mathrm{~d} s_{2}-\int_{\widehat{\omega}_{\varepsilon}^{*}} \mathcal{M}_{\varepsilon}(F) \cdot \mathcal{M}_{\varepsilon}\left(\mathcal{U}_{E}\right) \mathrm{d} s_{1} \mathrm{~d} s_{2}\right| \leq C \varepsilon\left\|\mathcal{U}_{E}\right\|_{H^{1}(\omega)}\|F\|_{H^{1}(\Omega)} \leq \frac{C}{\varepsilon^{1 / 2}}\|F\|_{H^{1}(\omega)}\|e(u)\|_{L^{2}\left(\mathcal{Q}_{\varepsilon}^{*}\right)} .
$$

Since $\mathcal{M}_{\varepsilon}(F) \cdot \mathcal{M}_{\varepsilon}\left(\mathcal{U}_{E}\right)$ is constant on every $\varepsilon$-cell, that gives

$$
\int_{\widehat{\omega}_{\varepsilon}^{*}} \mathcal{M}_{\varepsilon}(F) \cdot \mathcal{M}_{\varepsilon}\left(\mathcal{U}_{E}\right) \mathrm{d} s_{1} \mathrm{~d} s_{2}=\frac{\left|Y^{\prime *}\right|}{\left|Y^{\prime}\right|} \int_{\widehat{\omega}_{\varepsilon}} \mathcal{M}_{\varepsilon}(F) \cdot \mathcal{M}_{\varepsilon}\left(\mathcal{U}_{E}\right) \mathrm{d} s_{1} \mathrm{~d} s_{2} .
$$

Proceeding as above, one shows that

$$
\left|\int_{\widehat{\omega}_{\varepsilon}} F \cdot \mathcal{U}_{E} \mathrm{~d} s_{1} \mathrm{~d} s_{2}-\int_{\widehat{\omega}_{\varepsilon}} \mathcal{M}_{\varepsilon}(F) \cdot \mathcal{M}_{\varepsilon}\left(\mathcal{U}_{E}\right) \mathrm{d} s_{1} \mathrm{~d} s_{2}\right| \leq \frac{C}{\varepsilon^{1 / 2}}\|F\|_{H^{1}(\omega)}\|e(u)\|_{L^{2}\left(\mathcal{Q}_{\varepsilon}^{*}\right)} .
$$

Summarizing the above estimates and using (6.7) give (recall that there are no holes in $\Lambda_{\varepsilon}$ )

$$
\begin{aligned}
\left|\int_{\omega_{\varepsilon}^{*}} F \cdot \mathcal{U}_{E} \mathrm{~d} s_{1} \mathrm{~d} s_{2}-\frac{\left|Y^{\prime *}\right|}{\left|Y^{\prime}\right|} \int_{\omega} F \cdot \mathcal{U}_{E} \mathrm{~d} s_{1} \mathrm{~d} s_{2}\right| & \leq \frac{C}{\varepsilon^{1 / 2}}\|F\|_{H^{1}(\omega)}\|e(u)\|_{L^{2}\left(\mathcal{Q}_{\varepsilon}^{*}\right)}, \\
\text { and }\left|\int_{\omega} F \cdot \mathcal{U}_{E} \mathrm{~d} s_{1} \mathrm{~d} s_{2}\right| & \leq \frac{C}{\varepsilon^{1 / 2}}\left(\left\|F_{3}\right\|_{L^{2}\left(0, L ; H^{2}(0, a \pi)\right)}+\|F\|_{H^{1}(\omega)}\right)\|e(u)\|_{L^{2}\left(\mathcal{Q}_{\varepsilon}^{*}\right)}
\end{aligned}
$$

which leads to (6.10). 
Using now $u=u_{\varepsilon}$ as test function in (6.5) we obtain

$$
\left\|e\left(u_{\varepsilon}\right)\right\|_{L^{2}\left(\mathcal{Q}_{\varepsilon}^{*}\right)} \leq C \varepsilon^{3 / 2}\left(\|f\|_{L^{2}(\omega)}+\|g\|_{L^{2}(\omega)}+\left\|F_{3}\right\|_{L^{2}\left(0, L ; H^{2}(0, a \pi)\right)}+\|F\|_{H^{1}(\omega)}\right) .
$$

\section{UNFOLDED LIMIT PROBLEMS}

For every $\left(\mathcal{V}_{E}, \mathcal{V}\right)$ in $\mathbb{D}_{E} \times \mathbb{D}_{I}$ we define the symmetric tensor $\mathcal{E}\left(\mathcal{V}_{E}, \mathcal{V}\right)$ by

$$
\mathcal{E}\left(\mathcal{V}_{E}, \mathcal{V}\right)=\left(\begin{array}{ccc}
\mathcal{Z}_{11}\left(\mathcal{V}_{E}\right)-y_{3} \Lambda_{11}(\mathcal{V}) & \mathcal{Z}_{12}\left(\mathcal{V}_{E}\right)-y_{3} \Lambda_{12}(\mathcal{V}) & 0 \\
\mathcal{Z}_{12}\left(\mathcal{V}_{E}\right)-y_{3} \Lambda_{12}(\mathcal{V}) & \mathcal{Z}_{22}\left(\mathcal{V}_{E}\right)-y_{3} \Lambda_{22}(\mathcal{V}) & 0 \\
0 & 0 & 0
\end{array}\right)
$$

with

$$
\mathcal{Z}_{\alpha \beta}(\mathcal{V})=\frac{1}{2}\left[\frac{\partial \mathcal{V}}{\partial s_{\alpha}} \mathbf{t}_{\beta}+\frac{\partial \mathcal{V}}{\partial s_{\beta}} \mathbf{t}_{\alpha}\right]
$$

and

$$
\begin{aligned}
& \Lambda_{11}(\mathcal{V})=\frac{\partial^{2} \mathcal{V}_{3}}{\partial s_{1}^{2}}-\frac{1}{a} \frac{\partial \mathcal{V}_{1}}{\partial s_{1}}=\frac{\partial}{\partial s_{1}}\left(\frac{\partial \mathcal{V}}{\partial s_{1}} \mathbf{n}\right), \quad \Lambda_{22}(\mathcal{V})=\frac{\partial^{2} \mathcal{V}_{3}}{\partial s_{2}^{2}}=\frac{\partial}{\partial s_{2}}\left(\frac{\partial \mathcal{V}}{\partial s_{2}} \mathbf{n}\right) \\
& \Lambda_{12}(\mathcal{V})=\frac{\partial^{2} \mathcal{V}_{3}}{\partial s_{1} \partial s_{2}}-\frac{1}{a} \frac{\partial \mathcal{V}_{1}}{\partial s_{2}}=\frac{\partial}{\partial s_{2}}\left(\frac{\partial \mathcal{V}}{\partial s_{1}} \mathbf{n}\right) .
\end{aligned}
$$

Denote $H_{\text {per }}^{1}\left(Y^{*}\right)$ the subspace of $H^{1}\left(Y^{*}\right)$ containing the functions $\mathbf{G}$ periodic and

$$
\mathbb{D} \doteq\left\{v=\left(\mathcal{V}_{E}, \mathcal{V}, \widehat{v}\right) \in \mathbb{D}_{I} \times \mathbb{D}_{E} \times L^{2}\left(\Omega ; H_{\mathrm{per}}^{1}\left(Y^{*}\right)\right)^{3}\right\} .
$$

For every $v \in \mathbb{D}$ we consider the symmetric tensor

$$
\mathcal{E}\left(\mathcal{V}_{E}, \mathcal{V}\right)+\mathcal{E}_{y}(\widehat{v})
$$

and the semi-norm

$$
\|v\|_{\mathbb{D}}=\left\|\mathcal{E}\left(\mathcal{V}_{E}, \mathcal{V}\right)+\mathcal{E}_{y}(\widehat{v})\right\|_{L^{2}\left(\omega \times Y^{*}\right)} .
$$

Lemma 7.1. Given the expressions (3.7) for $\mathcal{V} \in \mathbb{D}_{I}$, there exist $c, C \in \mathbb{R}_{+}$such that

$$
c\|\mathcal{V}\|_{\mathbb{D}_{I}}^{2} \leq \sum_{\alpha, \beta=1}^{2}\left\|\Lambda_{\alpha \beta}(\mathcal{V})\right\|_{L^{2}(\omega)}^{2} \leq C\|\mathcal{V}\|_{\mathbb{D}_{I}}^{2}
$$

Proof. First, one has

$$
\sum_{\alpha, \beta=1}^{2}\left\|\Lambda_{\alpha \beta}(\mathcal{V})\right\|_{L^{2}(\omega)}^{2} \leq C\left(\left\|D_{2} \mathcal{V}_{3}\right\|_{L^{2}(\omega)}+\left\|\nabla \mathcal{V}_{1}\right\|_{L^{2}(\omega)}\right)
$$

This inequality and Lemma 5.1 give the inequality in the right-hand side.

We prove the left-hand side of the inequality by contradiction. We assume that there exists a sequence $\left(\mathcal{V}_{n}\right)_{n \in \mathbb{N}}$ in $\mathbb{D}_{I}$, such that

$$
\left\|\mathcal{V}_{n}\right\|_{\mathbb{D}_{I}}=1, \sum_{\alpha, \beta=1}^{2}\left\|\Lambda_{\alpha \beta}\left(\mathcal{V}_{n}\right)\right\|_{L^{2}(\omega)}^{2} \rightarrow 0 \quad \text { as } \quad n \rightarrow \infty .
$$

By Lemma 5.1 and the expressions in (5.1), we can also consider a sequence $\left(V_{1, n}, V_{2, n}\right)_{n \in \mathbb{N}}$ in $H_{0}^{3}(0, a \pi) \times$ $H_{0}^{4}(0, a \pi)$ with

$$
\left\|V_{1, n}\right\|_{H_{0}^{3}(0, a \pi)}^{2}+\left\|V_{2, n}\right\|_{H_{0}^{4}(0, a \pi)}^{2}=1
$$


and the components $\Lambda_{\alpha \beta}$ can be expressed as

$$
\begin{aligned}
& \Lambda_{11}\left(\mathcal{V}_{n}\right)=\frac{1}{a}\left(s_{2}^{c} V_{2, n}^{\prime \prime}\left(s_{1}\right)-V_{1, n}^{\prime}\left(s_{1}\right)\right)+a\left(s_{2}^{c} V_{2, n}^{\prime \prime \prime \prime}\left(s_{1}\right)-V_{1, n}^{\prime \prime \prime}\right), \\
& \Lambda_{12}\left(\mathcal{V}_{n}\right)=\frac{1}{a} V_{2, n}^{\prime}\left(s_{1}\right)+a V_{2, n}^{\prime \prime \prime}\left(s_{1}\right), \quad \Lambda_{22}\left(\mathcal{V}_{n}\right)=0 .
\end{aligned}
$$

We have then that there exists $\left(V_{1}, V_{2}\right)$ in $H_{0}^{3}(0, a \pi) \times H_{0}^{4}(0, a \pi)$ such that

$$
\left(V_{1, n}, V_{2, n}\right) \rightarrow\left(V_{1}, V_{2}\right) \quad \text { weakly in } H_{0}^{3}(0, a \pi) \times H_{0}^{4}(0, a \pi) .
$$

By Sobolev embedding we then get

$$
\left(V_{1, n}, V_{2, n}\right) \longrightarrow\left(V_{1}, V_{2}\right) \text { strongly } \quad \text { in } H_{0}^{2}(0, a \pi) \times H_{0}^{3}(0, a \pi) .
$$

Moreover, since $\left\|\Lambda_{\alpha \beta}\right\| \longrightarrow 0$ for $(\alpha, \beta) \in\{(1,1),(1,2),(2,2)\}$ we have that

$$
\frac{1}{a}\left(s_{2}^{c} V_{2}^{\prime \prime}\left(s_{1}\right)-V_{1}^{\prime}\left(s_{1}\right)\right)+a\left(s_{2}^{c} V_{2}^{\prime \prime \prime \prime}\left(s_{1}\right)-V_{1}^{\prime \prime \prime}\right)=0, \quad \frac{1}{a} V_{2}^{\prime}\left(s_{1}\right)+a V_{2}^{\prime \prime \prime}\left(s_{1}\right)=0 .
$$

Solving the differential equations with the respective boundary conditions we obtain that $V_{2}=V_{1}=0$. Therefore, we have that $\left(V_{1, n}, V_{2, n}\right)$ converges strongly to $(0,0)$ in $H_{0}^{2}(0, a \pi) \times H_{0}^{3}(0, a \pi)$.

Considering again equation (7.1) and with our assumption that $\left\|\Lambda_{11}\left(\mathcal{V}_{n}\right)\right\|_{L^{2}(\omega)} \rightarrow 0$, we also get $\left(V_{1, n}^{\prime \prime \prime}, V_{2, n}^{\prime \prime \prime \prime}\right) \rightarrow$ $(0,0)$ strongly in $L^{2}(0, a \pi)^{2}$ and then the convergence $\left(V_{1, n}, V_{2, n}\right) \rightarrow(0,0)$ strongly in $H_{0}^{3}(0, a \pi) \times H_{0}^{4}(0, a \pi)$, which contradicts the fact that $\left\|V_{1}\right\|_{H_{0}^{3}(0, a \pi)}^{2}+\left\|V_{2}\right\|_{H_{0}^{4}(0, a \pi)}^{2}=1$ coming from the assumption $\left\|V_{1, n}\right\|_{H_{0}^{3}(0, a \pi)}^{2}+$ $\left\|V_{2, n}\right\|_{H_{0}^{4}(0, a \pi)}^{2}=1$ for all $n \in \mathbb{N}$.

Lemma 7.2. Consider the space $\mathbb{S} \doteq \mathbb{R}^{3} \times \mathbb{R}^{3} \times H_{\text {per }, 0}^{1}\left(Y^{*}\right)^{3}$ with the seminorm

$$
\begin{aligned}
\left\|\left(\tau_{A}, \tau_{B}, \widehat{w}\right)\right\|_{\mathbb{S}}^{2}= & \sum_{\substack{\alpha, \beta=1 \\
\alpha \leq \beta}}^{2}\left\|\tau_{A}^{\alpha \beta}+y_{3} \tau_{B}^{\alpha \beta}+e_{\alpha \beta, y}(\widehat{w})\right\|_{L^{2}\left(Y^{*}\right)}^{2} \\
& +\left\|e_{13, y}(\widehat{w})\right\|_{L^{2}\left(Y^{*}\right)}^{2}+\left\|e_{23, y}(\widehat{w})\right\|_{L^{2}\left(Y^{*}\right)}^{2}+\left\|e_{33, y}(\widehat{w})\right\|_{L^{2}\left(Y^{*}\right)}^{2} .
\end{aligned}
$$

Then this expression actually defines a norm on $\mathbb{S}$ equivalent to the product-norm.

Proof. We consider the field $\Phi \in H^{1}\left(\mathbb{R}^{3}\right)^{3}$ given by

$$
\begin{aligned}
& \Phi_{1}(y)=y_{1}\left(\tau_{A}^{11}+y_{3} \tau_{B}^{11}\right)+y_{2}\left(\tau_{A}^{12}+y_{3} \tau_{B}^{12}\right), \\
& \Phi_{2}(y)=y_{2}\left(\tau_{A}^{22}+y_{3} \tau_{B}^{22}\right)+y_{1}\left(\tau_{A}^{12}+y_{3} \tau_{B}^{12}\right), \\
& \Phi_{3}(y)=-\left[\frac{\left(y_{1}\right)^{2}}{2} \tau_{B}^{11}+\frac{\left(y_{2}\right)^{2}}{2} \tau_{B}^{22}+y_{2} y_{1} \tau_{B}^{12}\right] .
\end{aligned}
$$

Hence, we have

$$
\left\|\left(\tau_{A}, \tau_{B}, \widehat{w}\right)\right\|_{\mathbb{S}}=\left\|\mathcal{E}_{y}(\Phi+\widehat{w})\right\|_{L^{2}\left(Y^{*}\right)} .
$$

We will now show that $\left\|\mathcal{E}_{y}(\Phi+\widehat{w})\right\|_{L^{2}\left(Y^{*}\right)}=0 \Longrightarrow \Phi=0, \widehat{w}=0$.

Consider the case that $\mathcal{E}_{y}(\Phi+\widehat{w})=0$, which yields that $\Phi+\widehat{w}$ is a rigid displacement. Hence, there exist $a, b \in \mathbb{R}^{3}$ such that

$$
\Phi+\widehat{w}=r, \quad r(y)=\left(\begin{array}{l}
a_{1}+b_{2} y_{3}-b_{3} y_{2} \\
a_{2}+b_{3} y_{1}-b_{1} y_{3} \\
a_{3}+b_{1} y_{2}-b_{2} y_{1}
\end{array}\right) .
$$


Since, $\widehat{w}$ is a periodic function with period $\mathbf{p}_{1}, \mathbf{p}_{2}$, one has $(\Phi-r)\left(y+\mathbf{p}_{i}\right)=(\Phi-r)(y)$ for a.e. $y \in\left(\mathbb{R}^{2} \backslash\right.$ $\left.\bigcup_{\xi \in \mathbf{G}}(\xi+\bar{S})\right) \times(-\kappa, \kappa)$. Considering the first two components yields the equations

$$
\begin{array}{ll}
\tau_{A}^{11}+y_{3} \tau_{B}^{11}=0, & \tau_{A}^{12}+y_{3} \tau_{B}^{12}=-b_{3}, \\
\tau_{A}^{22}+y_{3} \tau_{B}^{22}=0, & \tau_{A}^{12}+y_{3} \tau_{B}^{12}=b_{3} .
\end{array}
$$

Therefore, we obtain $\tau_{A}^{11}=\tau_{B}^{11}=\tau_{A}^{22}=\tau_{B}^{22}=\tau_{A}^{12}=\tau_{B}^{12}=0$ and $b_{3}=0$. Now, the equality of the third component yields $b_{1}=b_{2}=0$. Finally, we conclude that $\Phi=0, r$ is a constant displacement and since $\widehat{w} \in H_{\text {per }, 0}^{1}\left(Y^{*}\right)^{3}$ the displacement $r=0$ and therefore $\widehat{w}=0$, which proves that $\|\cdot\|_{\mathbb{S}}$ is a norm.

By contradiction we easily prove that there exists a constant $C>0$ such that

$$
\left.C\left(\left|\tau_{A}\right|+\left|\tau_{B}\right|+\|\widehat{w}\|_{H^{1}\left(Y^{*}\right)}\right) \leq\left\|\left(\tau_{A}, \tau_{B}, \widehat{w}\right)\right\|_{\mathbb{S}}, \quad \forall\left(\tau_{A}, \tau_{B}, \widehat{w}\right)\right) \in \mathbb{S}
$$

which ends the proof.

Lemma 7.3. The semi-norm $\|\cdot\|_{\mathbb{D}}$ is a norm equivalent to the product-norm of $\mathbb{D}_{I} \times \mathbb{D}_{E} \times L^{2}\left(\Omega ; H_{\mathrm{per}}^{1}\left(Y^{*}\right)\right)^{3}$.

Proof. By the definition of $\|\cdot\|_{\mathbb{D}_{I}}$, we have that

$$
\begin{aligned}
\|v\|_{\mathbb{D}}^{2}= & \sum_{\alpha, \beta=1}^{2}\left\|\mathcal{Z}_{\alpha \beta}\left(\mathcal{V}_{E}\right)-y_{3} \Lambda_{\alpha \beta}(\mathcal{V})+e_{\alpha \beta, y}(\widehat{v})\right\|_{L^{2}\left(\omega \times Y^{*}\right)}^{2} \\
& +2\left\|e_{13, y}(\widehat{v})\right\|_{L^{2}\left(\omega \times Y^{*}\right)}^{2}+2\left\|e_{23, y}(\widehat{v})\right\|_{L^{2}\left(\omega \times Y^{*}\right)}^{2}+2\left\|e_{33, y}(\widehat{v})\right\|_{L^{2}\left(\omega \times Y^{*}\right)}^{2} .
\end{aligned}
$$

We may further note that we have

$$
\begin{aligned}
\left\|\mathcal{E}\left(\mathcal{V}_{E}, \mathcal{V}\right)\right\|_{L^{2}(\Omega)}^{2} & =\sum_{\alpha, \beta=1}^{2} \int_{\Omega}\left(Z_{\alpha \beta}\left(\mathcal{V}_{E}\right)+y_{3} \Lambda_{\alpha \beta}\left(\mathcal{V}_{I}\right)\right)^{2} \mathrm{~d} s \\
& =2 \kappa \sum_{\alpha, \beta=1}^{2}\left\|\mathcal{Z}_{\alpha \beta}\left(\mathcal{V}_{E}\right)\right\|_{L^{2}(\omega)}^{2}+\frac{2 \kappa^{3}}{3} \sum_{\alpha, \beta=1}^{2}\left\|\Lambda_{\alpha \beta}(\mathcal{V})\right\|_{L^{2}(\omega)}^{2} .
\end{aligned}
$$

We obtain with Lemma 7.2 and the equivalence of norms that

$$
\begin{gathered}
c\left(\sum_{\alpha, \beta=1}^{2}\left\|\mathcal{Z}_{\alpha \beta}\right\|_{L^{2}(\omega)}+\sum_{\alpha, \beta=1}^{2}\left\|\Lambda_{\alpha \beta}\right\|_{L^{2}(\omega)}+\|\widehat{v}\|_{L^{2}\left(\omega \times Y^{*}\right)}\right) \leq\|\mathcal{V}\|_{\mathbb{D}_{I}} \\
\leq C\left(\sum_{\alpha, \beta=1}^{2}\left\|\mathcal{Z}_{\alpha \beta}\right\|_{L^{2}(\omega)}+\sum_{\alpha, \beta=1}^{2}\left\|\Lambda_{\alpha \beta}\right\|_{L^{2}(\omega)}+\|\widehat{v}\|_{L^{2}\left(\omega \times Y^{*}\right)}\right) .
\end{gathered}
$$

Further note that $\sum_{\alpha, \beta=1}^{2}\left\|\mathcal{Z}_{\alpha \beta}\left(\mathcal{V}_{E}\right)\right\|_{L^{2}(\omega)}^{2}=\left\|\mathcal{V}_{E}\right\|_{E}^{2}$.

Besides, with Lemma 7.1 we obtain that

$$
c\|\mathcal{V}\|_{\mathbb{D}_{I}}^{2} \leq \sum_{\alpha, \beta=1}^{2}\left\|\Lambda_{\alpha \beta}(\mathcal{V})\right\|_{L^{2}(\omega)}^{2} \leq C\|\mathcal{V}\|_{\mathbb{D}_{I}}^{2} .
$$

Finally, we can conclude

$$
c\left(\left\|\mathcal{V}_{E}\right\|_{E}+\|\mathcal{V}\|_{\mathbb{D}_{I}}+\|\widehat{v}\|_{L^{2}\left(\omega \times Y^{*}\right)}\right) \leq\|v\|_{\mathbb{D}} \leq C\left(\left\|\mathcal{V}_{E}\right\|_{E}+\|\mathcal{V}\|_{\mathbb{D}_{I}}+\|\widehat{v}\|_{L^{2}\left(\omega \times Y^{*}\right)}\right) .
$$


Theorem 7.4. Let $u_{\varepsilon}$ be the solution of the elasticity problem (6.5). Then the following convergence holds:

$$
\frac{1}{\varepsilon} \Pi_{\varepsilon}\left(e\left(u_{\varepsilon}\right)\right) \rightarrow \mathcal{E}\left(\mathcal{U}_{E}, \mathcal{U}\right)+\mathcal{E}_{y}(\widehat{u}) \quad \text { strongly } \quad \text { in } \quad L^{2}\left(\omega \times Y^{*}\right)^{9},
$$

where $\left(\mathcal{U}_{E}, \mathcal{U}, \widehat{u}\right) \in \mathbb{D}$ is the unique solution of the rescaled and unfolded problem

$$
\begin{aligned}
& \frac{1}{2 \kappa} \int_{\omega \times Y^{*}} a_{i j k l}\left(E_{S, i j}\left(\mathcal{U}_{E}, \mathcal{U}\right)+\mathcal{E}_{i j, y}(\widehat{u})\right)\left(E_{S, k l}\left(\mathcal{V}_{E}, \mathcal{V}\right)+\mathcal{E}_{k l, y}(\widehat{v})\right) \mathrm{d} s^{\prime} \mathrm{d} y \\
& \quad=\left|Y^{\prime *}\right|\left(\int_{\omega}\left(f \cdot \mathcal{V}+\frac{\kappa^{2}}{3 a} g_{\alpha} \mathcal{V}_{\alpha}-\frac{\kappa^{2}}{3} g_{\alpha} \frac{\partial \mathcal{V}}{\partial s_{\alpha}} \boldsymbol{n}\right) \mathrm{d} s^{\prime}+\left\langle F, \mathcal{V}_{E}\right\rangle\right), \quad \forall\left(\mathcal{V}_{E}, \mathcal{V}, \widehat{v}\right) \in \mathbb{D} .
\end{aligned}
$$

Proof. Take $v=\left(\mathcal{V}_{E}, \mathcal{V}, \widehat{v}\right)$ such that

$$
\mathcal{V}_{E} \in \mathcal{C}^{1}(\bar{\omega})^{3} \cap \mathbb{D}_{E}, \quad \mathcal{V} \in \mathcal{C}^{2}(\bar{\omega})^{3} \cap \mathbb{D}_{I},
$$

and consider the test function $v_{\varepsilon}=v_{\varepsilon, 1}+v_{\varepsilon, 2}$, where

$$
\begin{aligned}
& v_{\varepsilon, 1}(s)=\mathcal{V}\left(s^{\prime}\right)+\varepsilon \mathcal{V}_{E}\left(s^{\prime}\right)-s_{3}\left[\frac{\partial\left(\mathcal{V}+\varepsilon \mathcal{V}_{E}\right)}{\partial s_{\alpha}}\left(s^{\prime}\right) \cdot \mathbf{n}\left(s^{\prime}\right)\right] \mathbf{t}_{\alpha}\left(s^{\prime}\right), \quad \text { for } \quad \text { a.e. } \quad s \in \Omega_{\varepsilon} \\
& v_{\varepsilon, 2}(s)=\varepsilon^{2} \widehat{v}\left(s^{\prime},\left\{\frac{s}{\varepsilon}\right\}\right),
\end{aligned}
$$

with $\widehat{v} \in \mathcal{C}^{1}\left(\bar{\omega} ; H_{\text {per }}^{1}\left(Y^{*}\right)^{3}\right)$ satisfying $\widehat{v}\left(0, s_{2}, y\right)=\widehat{v}\left(a \pi, s_{2}, y\right)$ for a.e. $\left(s_{2}, y\right) \in(0, L) \times Y^{*}$.

We calculate the elements $e_{12}\left(v_{\varepsilon, 1}\right)$ and $e_{13}\left(v_{\varepsilon, 1}\right)$, since the rest follows in a similar way. We obtain

$$
\begin{aligned}
e_{12}\left(v_{\varepsilon, 1}\right)= & \frac{1}{2} \frac{a}{a+s_{3}}\left[\frac{\partial \mathcal{V}_{2}}{\partial s_{1}}+\varepsilon \frac{\partial \mathcal{V}_{E, 2}}{\partial s_{1}}-s_{3}\left(\frac{\partial^{2} \mathcal{V}_{3}}{\partial s_{1} \partial s_{2}}+\varepsilon \frac{\partial^{2} \mathcal{V}_{E, 3}}{\partial s_{1} \partial s_{2}}\right)\right. \\
& \left.+\left(1+\frac{s_{3}}{a}\right)\left(\frac{\partial \mathcal{V}_{1}}{\partial s_{2}}+\varepsilon \frac{\partial \mathcal{V}_{E, 1}}{\partial s_{2}}\right)-\left(s_{3}+\frac{s_{3}^{2}}{a}\right)\left(\frac{\partial^{2} \mathcal{V}_{3}}{\partial s_{1} \partial s_{2}}-\frac{1}{a} \frac{\partial \mathcal{V}_{1}}{\partial s_{2}}+\varepsilon\left(\frac{\partial^{2} \mathcal{V}_{E, 3}}{\partial s_{1} \partial s_{2}}-\frac{1}{a} \frac{\partial \mathcal{V}_{E, 1}}{\partial s_{2}}\right)\right)\right] .
\end{aligned}
$$

Applying the rescaling-unfolding operator $\Pi_{\varepsilon}$ and dividing by $\varepsilon$ yields with the properties for $\mathbb{D}_{I}$ that

$$
\begin{aligned}
\frac{1}{\varepsilon} \Pi_{\varepsilon}\left(e_{12}\left(v_{\varepsilon, 1}\right)\right)= & \frac{1}{2} \frac{a}{a+\varepsilon y_{3}}\left[\left(\frac{\partial \mathcal{V}_{E, 2}}{\partial s_{1}}+\frac{\partial \mathcal{V}_{E, 1}}{\partial s_{2}}\right)-2 y_{3} \frac{\partial^{2} \mathcal{V}_{3}}{\partial s_{1} \partial s_{2}}+2 \frac{y_{3}}{a} \frac{\partial \mathcal{V}_{1}}{\partial s_{2}}\right. \\
& \left.+\frac{\varepsilon y_{3}}{a} \frac{\partial \mathcal{V}_{E, 1}}{\partial s_{2}}-\frac{\varepsilon y_{3}}{a} \frac{\partial^{2} \mathcal{V}_{3}}{\partial s_{1} \partial s_{2}}+\frac{\varepsilon y_{3}}{a} \frac{\partial \mathcal{V}_{1}}{\partial s_{2}}-\varepsilon y_{3} \frac{\partial^{2} \mathcal{V}_{E, 3}}{\partial s_{1} \partial s_{2}}-\left(\varepsilon+\frac{\varepsilon^{2}}{a}\right)\left(\frac{\partial^{2} \mathcal{V}_{E, 3}}{\partial s_{1} \partial s_{2}}-\frac{1}{a} \frac{\partial \mathcal{V}_{E, 1}}{\partial s_{2}}\right)\right] \\
& \longrightarrow \frac{1}{2} \mathcal{Z}_{12}\left(\mathcal{V}_{E}\right)-y_{3}\left(\frac{\partial^{2} \mathcal{V}_{3}}{\partial s_{1} \partial s_{2}}-\frac{\partial \mathcal{V}_{1}}{\partial s_{2}}\right) \quad \text { strongly } \quad \text { in } \quad L^{2}\left(\omega \times Y^{*}\right)^{9} .
\end{aligned}
$$

For $e_{13}\left(v_{\varepsilon, 1}\right)$ we then obtain

$$
\begin{aligned}
e_{13}\left(v_{\varepsilon, 1}\right)= & \frac{1}{2} \frac{a}{a+s_{3}}\left[\left(\frac{\partial \mathcal{V}_{3}}{\partial s_{1}}-\frac{1}{a} \mathcal{V}_{1}\right)+\varepsilon\left(\frac{\partial \mathcal{V}_{E, 3}}{\partial s_{1}}-\frac{1}{a} \mathcal{V}_{1}\right)+\frac{s_{3}}{a}\left(\frac{\partial \mathcal{V}_{3}}{\partial s_{1}}-\frac{1}{a} \mathcal{V}_{1}\right)\right. \\
& \left.+\frac{s_{3} \varepsilon}{a}\left(\frac{\partial \mathcal{V}_{E, 3}}{\partial s_{1}}-\frac{1}{a} \mathcal{V}_{1}\right)+\left(1+\frac{s_{3}}{a}\right)\left(-\left(\frac{\partial \mathcal{V}_{3}}{\partial s_{1}}-\frac{1}{a} \mathcal{V}_{1}\right)-\varepsilon\left(\frac{\partial \mathcal{V}_{E, 3}}{\partial s_{1}}-\frac{1}{a} \mathcal{V}_{1}\right)\right)\right]=0
\end{aligned}
$$

In conclusion we get that

$$
\frac{1}{\varepsilon} \Pi_{\varepsilon}\left(e\left(v_{\varepsilon, 1}\right)\right) \longrightarrow \mathcal{E}\left(\mathcal{V}_{E}, \mathcal{V}\right) \quad \text { strongly } \quad \text { in } \quad L^{2}\left(\omega \times Y^{*}\right)^{9} .
$$


In the next step we focus on the calculation for $e_{i j}\left(v_{\varepsilon, 2}\right)$, where we once again just focus on $e_{12}$ and $e_{13}$. One has

$$
\begin{aligned}
& e_{12}\left(v_{\varepsilon, 2}\right)(s)=\frac{\varepsilon^{2}}{2}\left(\frac{a}{a+s_{3}} \frac{\partial \widehat{v}}{\partial s_{1}} \mathbf{t}_{2}+\frac{\partial \widehat{v}}{\partial s_{2}} \mathbf{t}_{1}\right)\left(s^{\prime},\left\{\frac{s}{\varepsilon}\right\}\right)+\frac{\varepsilon}{2}\left(\frac{a}{a+s_{3}} \frac{\partial \widehat{v}}{\partial y_{1}} \mathbf{t}_{2}+\frac{\partial \widehat{v}}{\partial y_{2}} \mathbf{t}_{1}\right)\left(s^{\prime},\left\{\frac{s}{\varepsilon}\right\}\right), \\
& e_{13}\left(v_{\varepsilon, 2}\right)(s)=\frac{\varepsilon^{2}}{2}\left(\frac{a}{a+s_{3}} \frac{\partial \widehat{v}}{\partial s_{1}} \mathbf{n}+\frac{\partial \widehat{v}}{\partial s_{3}} \mathbf{t}_{1}\right)\left(s^{\prime},\left\{\frac{s}{\varepsilon}\right\}\right)+\frac{\varepsilon}{2}\left(\frac{a}{a+s_{3}} \frac{\partial \widehat{v}}{\partial y_{1}} \mathbf{n}+\frac{\partial \widehat{v}}{\partial y_{3}} \mathbf{t}_{1}\right)\left(s^{\prime},\left\{\frac{s}{\varepsilon}\right\}\right) .
\end{aligned}
$$

Considering now $\frac{1}{\varepsilon} \Pi_{\varepsilon}\left(e_{12}\left(v_{\varepsilon, 2}\right)\right)$ and $\frac{1}{\varepsilon} \Pi_{\varepsilon}\left(e_{13}\left(v_{\varepsilon, 2}\right)\right)$, we obtain

$$
\begin{aligned}
& \frac{1}{\varepsilon} \Pi_{\varepsilon}\left(e_{12}\left(v_{\varepsilon, 2}\right)\right) \longrightarrow e_{y, 12}(\widehat{v}) \quad \text { strongly } \text { in } L^{2}\left(\omega \times Y^{*}\right), \\
& \frac{1}{\varepsilon} \Pi_{\varepsilon}\left(e_{13}\left(v_{\varepsilon, 2}\right)\right) \longrightarrow e_{y, 13}(\widehat{v}) \quad \text { strongly } \text { in } L^{2}\left(\omega \times Y^{*}\right),
\end{aligned}
$$

which then yields

$$
\frac{1}{\varepsilon} \Pi_{\varepsilon}\left(e\left(v_{\varepsilon, 2}\right)\right) \longrightarrow \mathcal{E}_{y}(\widehat{v}) \quad \text { strongly } \quad \text { in } \quad L^{2}\left(\omega \times Y^{*}\right)^{9},
$$

therefore

$$
\frac{1}{\varepsilon} \Pi_{\varepsilon}\left(e\left(v_{\varepsilon}\right)\right) \longrightarrow \mathcal{E}\left(\mathcal{V}_{E}, \mathcal{V}\right)+\mathcal{E}_{y}(\widehat{v}) \quad \text { strongly } \quad \text { in } \quad L^{2}\left(\omega \times Y^{*}\right)^{9}
$$

Plugging in our test function $v_{\varepsilon}$ into the weak formulation (6.5), applying the rescaling-unfolding operator on both sides. Dividing by $2 \kappa \varepsilon^{3}$ and passing to the limit, we obtain (7.4) with the chosen test functions (regarding the right-hand side, we use the results from Lem. 6.1 and [10], Prop. 4.8 to get an integral over the whole domain $\omega$ at the limit). Then, by density of $\mathcal{C}^{1}(\bar{\omega})^{3} \cap \mathbb{D}_{E}$ in $\mathbb{D}_{E}, \mathcal{C}^{2}(\bar{\omega})^{3} \cap \mathbb{D}_{I}$ in $\mathbb{D}_{I}$ and $\mathcal{C}^{1}\left(\bar{\omega} ; H_{\text {per }}^{1}\left(Y^{*}\right)^{3}\right)$ in $L^{2}\left(\omega ; H_{\text {per }}^{1}\left(Y^{*}\right)^{3}\right)$, this yields $(7.4)$ for every $\left(\mathcal{V}_{E}, \mathcal{V}, \widehat{v}\right) \in \mathbb{D}$.

Due to the coercivity of $a_{i j k l}$ and Lemma A.1, we can apply Lax-Milgram theorem to the weak formulation (7.4). Therefore, this problem has an unique solution.

\section{HomogenizATion OF THE SHELL}

In this section we want to express the warping-microscopic displacement $\widehat{u}$ with respect to the macroscopic $\mathcal{U}_{E}$ and $\mathcal{U}$. Therefore, choosing $\mathcal{V}=0$ in equation (6.5) leads to

$$
\int_{Y^{*}} a_{i j k l}\left(E_{S, i j}\left(\mathcal{U}_{E}, \mathcal{U}\right)+\mathcal{E}_{i j, y}(\widehat{u})\right) \mathcal{E}_{k l, y}(\widehat{v}) \mathrm{d} y=0 \quad \forall \widehat{v} \in H_{\text {per }}^{1}\left(Y^{*}\right)^{3} .
$$

Hence, we can write $\widehat{u}$ in terms of $\left(\mathcal{U}_{E}, \mathcal{U}\right)$. We define the 3 matrices

$$
\mathbf{M}^{11}=\left(\begin{array}{lll}
1 & 0 & 0 \\
0 & 0 & 0 \\
0 & 0 & 0
\end{array}\right), \quad \mathbf{M}^{12}=\mathbf{M}^{21}=\left(\begin{array}{lll}
0 & 1 & 0 \\
1 & 0 & 0 \\
0 & 0 & 0
\end{array}\right), \quad \mathbf{M}^{22}=\left(\begin{array}{lll}
0 & 0 & 0 \\
0 & 1 & 0 \\
0 & 0 & 0
\end{array}\right),
$$

and introduce the 6 distinct correctors $\left((\alpha, \beta) \in\{1,2\}^{2}\right)$

$$
\begin{aligned}
& \widetilde{\chi_{E}^{\alpha \beta}} \in \widetilde{H_{\mathrm{per}}^{1}}\left(Y^{*}\right)^{3}, \quad \widetilde{\chi_{I}^{\alpha \beta}} \in H_{\mathrm{per}}^{1}\left(Y^{*}\right)^{3}, \quad \text { where } \\
& \widetilde{\chi_{E}^{12}}=\widetilde{\chi_{E}^{21}}, \quad \widetilde{\chi_{I}^{12}}=\widetilde{\chi_{I}^{21}},
\end{aligned}
$$

and which are defined by

$$
\begin{gathered}
\int_{Y^{*}} a_{i j k l}\left(\mathbf{M}_{i j}^{\alpha \beta}+\mathcal{E}_{i j, y}\left(\widetilde{\chi_{E}^{\alpha \beta}}\right)\right) \mathcal{E}_{k l, y}(\widetilde{\psi}) \mathrm{d} y=0, \quad \forall \widetilde{\psi} \in H_{\mathrm{per}}^{1}\left(Y^{*}\right)^{3}, \\
\int_{Y^{*}} a_{i j k l}\left(y_{3} \mathbf{M}_{i j}^{\alpha \beta}+\mathcal{E}_{i j, y}\left(\widetilde{\chi_{I}^{\alpha \beta}}\right)\right) \mathcal{E}_{k l, y}(\widetilde{\psi}) \mathrm{d} y=0 .
\end{gathered}
$$


Hence, we can write $\widehat{u}$ as

$$
\widehat{u}\left(s^{\prime}, y\right)=e_{\alpha \beta}\left(\mathcal{U}_{E}\right)\left(s^{\prime}\right) \widetilde{\chi_{E}^{\alpha \beta}}(y)+\Lambda_{\alpha \beta}(\mathcal{U})\left(s^{\prime}\right) \widetilde{\chi_{I}^{\alpha \beta}}(y) \text { for } \quad \text { a.e. } \quad\left(s^{\prime}, y\right) \in \omega \times Y^{*} .
$$

\subsection{The limit problems in the shell's mid surface}

Theorem 8.1. The limit displacement $\left(\mathcal{U}_{E}, \mathcal{U}\right) \in \mathbb{D}_{E} \times \mathbb{D}_{I}$ solves the homogenized problem

$$
\begin{aligned}
\int_{\omega}[ & a_{\alpha \beta \alpha^{\prime} \beta^{\prime}}^{\text {hom }} e_{\alpha \beta}\left(\mathcal{U}_{E}\right) e_{\alpha^{\prime} \beta^{\prime}}\left(\mathcal{V}_{E}\right)+b_{\alpha \beta \alpha^{\prime} \beta^{\prime}}^{\text {hom }}\left(e_{\alpha \beta}\left(\mathcal{U}_{E}\right) \Lambda_{\alpha^{\prime} \beta^{\prime}}(\mathcal{V})\right. \\
& \left.\left.+\Lambda_{\alpha \beta}(\mathcal{U}) e_{\alpha^{\prime} \beta^{\prime}}\left(\mathcal{V}_{E}\right)\right)+c_{\alpha \beta \alpha^{\prime} \beta^{\prime}}^{\text {hom }} \Lambda_{\alpha \beta}(\mathcal{U}) \Lambda_{\alpha^{\prime} \beta^{\prime}}(\mathcal{V})\right] \mathrm{d} s^{\prime} \\
= & \frac{\left|Y^{\prime *}\right|}{\left|Y^{\prime}\right|}\left(\int_{\omega}\left(f \cdot \mathcal{V}+\frac{\kappa^{2}}{3 a} g_{\alpha} \mathcal{V}_{\alpha}-\frac{\kappa^{2}}{3} g_{\alpha} \frac{\partial \mathcal{V}}{\partial s_{\alpha}} n\right) \mathrm{d} s^{\prime}+\left\langle F, \mathcal{V}_{E}\right\rangle\right), \quad \forall\left(\mathcal{V}_{E}, \mathcal{V}\right) \in \mathbb{D}_{E} \times \mathbb{D}_{I},
\end{aligned}
$$

where

$$
\begin{aligned}
& a_{\alpha \beta \alpha^{\prime} \beta^{\prime}}^{\mathrm{hom}}=\frac{1}{\left|Y^{*}\right|} \int_{Y^{*}} a_{i j k l}(y)\left[\boldsymbol{M}_{i j}^{\alpha \beta}+\mathcal{E}_{i j, y}\left(\widetilde{\chi_{E}^{\alpha \beta}}\right)\right] \boldsymbol{M}_{k l}^{\alpha^{\prime} \beta^{\prime}} \mathrm{d} y, \\
& b_{\alpha \beta \alpha^{\prime} \beta^{\prime}}^{\text {homm }}=\frac{1}{\left|Y^{*}\right|} \int_{Y^{*}} a_{i j k l}(y)\left[y_{3} \boldsymbol{M}_{i j}^{\alpha \beta}+\mathcal{E}_{i j, y}\left(\widetilde{\chi_{I}^{\alpha \beta}}\right)\right] \boldsymbol{M}_{k l}^{\alpha^{\prime} \beta^{\prime}} \mathrm{d} y, \\
& c_{\alpha \beta \alpha^{\prime} \beta^{\prime}}^{\text {hom }}=\frac{1}{\left|Y^{*}\right|} \int_{Y^{*}} a_{i j k l}(y)\left[y_{3} \boldsymbol{M}_{i j}^{\alpha \beta}+\mathcal{E}_{i j, y}\left(\widetilde{\chi_{I}^{\alpha \beta}}\right)\right] y_{3} \boldsymbol{M}_{k l}^{\alpha^{\prime} \beta^{\prime}} \mathrm{d} y .
\end{aligned}
$$

Proof. Consider equation (6.5) and choose the test function such that $\left(\mathcal{V}_{E}, \mathcal{V}\right) \in \mathbb{D}_{E} \times \mathbb{D}_{I}$ and $\widehat{v}=0$. Moreover, with the expression for $\widehat{u}$ we obtain for the left hand side in (6.5)

$$
\frac{1}{2 \kappa} \int_{\omega \times Y^{*}} a_{i j k l}(y)\left(E_{S, i j}\left(\mathcal{U}_{E}, \mathcal{U}\right)+\mathcal{E}_{i j, y}(\widehat{u})\right) E_{S, k l}\left(\mathcal{V}_{E}, \mathcal{V}\right) \mathrm{d} s^{\prime} \mathrm{d} y
$$

Hence,

$$
\begin{aligned}
\int_{\omega \times Y^{*}} & a_{i j k l}(y)\left[e_{\alpha \beta}\left(\mathcal{U}_{E}\right)\left(s^{\prime}\right)\left(\mathbf{M}_{i j}^{\alpha \beta}+\mathcal{E}_{i j, y}\left(\widetilde{\chi_{E}^{\alpha \beta}}\right)(y)\right)+\Lambda_{\alpha \beta}(\mathcal{U})\left(s^{\prime}\right)\left(y_{3} \mathbf{M}_{i j}^{\alpha \beta}+\mathcal{E}_{i j, y}\left(\widetilde{\chi_{I}^{\alpha \beta}}\right)(y)\right)\right] \\
& \times \mathbf{M}_{k l}^{\alpha^{\prime} \beta^{\prime}}\left[e_{\alpha^{\prime} \beta^{\prime}}\left(\mathcal{V}_{M}\right)\left(s^{\prime}\right)+y_{3} \Lambda_{\alpha^{\prime} \beta^{\prime}}(\mathcal{V})\left(s^{\prime}\right)\right] \mathrm{d} s^{\prime} \mathrm{d} y \\
= & \left|Y^{*}\right|\left(\int_{\omega}\left(f \cdot \mathcal{V}+\frac{\kappa^{2}}{3 a} g_{\alpha} \mathcal{V}_{\alpha}-\frac{\kappa^{2}}{3} g_{\alpha} \frac{\partial \mathcal{V}}{\partial s_{\alpha}} \mathbf{n}\right) \mathrm{d} s^{\prime}+\left\langle F, \mathcal{V}_{E}\right\rangle\right) .
\end{aligned}
$$

Computing the expression yields,

$$
\begin{aligned}
& \frac{1}{\left|Y^{*}\right|} \int_{\omega \times Y *} a_{i j k l}(y) e_{\alpha \beta}\left(\mathcal{U}_{E}\right)\left(\mathbf{M}_{i j}^{\alpha \beta}+\mathcal{E}_{i j, y}\left(\widetilde{\chi_{E}^{\alpha \beta}}\right)\right) \mathbf{M}_{k l}^{\alpha^{\prime} \beta^{\prime}} e_{\alpha^{\prime} \beta^{\prime}}\left(\mathcal{V}_{E}\right) \\
& \quad+a_{i j k l}(y) \Lambda_{\alpha \beta}(\mathcal{U})\left(y_{3} \mathbf{M}_{i j}^{\alpha \beta}+\mathcal{E}_{i j, y}\left(\widetilde{\chi_{I}^{\alpha \beta}}\right)\right) \mathbf{M}_{k l}^{\alpha^{\prime} \beta^{\prime}} e_{\alpha^{\prime} \beta^{\prime}}\left(\mathcal{V}_{E}\right) \\
& \quad+a_{i j k l}(y) e_{\alpha \beta}\left(\mathcal{U}_{E}\right)\left(\mathbf{M}_{i j}^{\alpha \beta}+\mathcal{E}_{i j, y}\left(\widetilde{\chi_{E}^{\alpha \beta}}\right)\right) y_{3} \mathbf{M}_{k l}^{\alpha^{\prime} \beta^{\prime}} \Lambda_{\alpha^{\prime} \beta^{\prime}}(\mathcal{V}) \\
& \quad+a_{i j k l}(y) \Lambda_{\alpha \beta}(\mathcal{U})\left(y_{3} \mathbf{M}_{i j}^{\alpha \beta}+\mathcal{E}_{i j, y}\left(\widetilde{\chi_{I}^{\alpha \beta}}\right)\right) y_{3} \mathbf{M}_{k l}^{\alpha^{\prime} \beta^{\prime}} \Lambda_{\alpha^{\prime} \beta^{\prime}}(\mathcal{V}) \mathrm{d} y \mathrm{~d} s^{\prime} \\
& =\frac{\left|Y^{\prime *}\right|}{\left|Y^{\prime}\right|}\left(\int_{\omega}\left(f \cdot \mathcal{V}+\frac{\kappa^{2}}{3 a} g_{\alpha} \mathcal{V}_{\alpha}-\frac{\kappa^{2}}{3} g_{\alpha} \frac{\partial \mathcal{V}}{\partial s_{\alpha}} \mathbf{n}\right) \mathrm{d} s^{\prime}+\left\langle F, \mathcal{V}_{E}\right\rangle\right) .
\end{aligned}
$$

With the expressions for the homogenized coefficients we end up with equation (8.2). 
Denote $\mathbb{S}_{2}$ the set of $2 \times 2$ symmetric matrices.

Lemma 8.2. There exists a constant $C>0$ such that the homogenized coefficients satisfy

$$
\begin{aligned}
a_{\alpha \beta \alpha^{\prime} \beta^{\prime}}^{\text {hom }} \tau_{E}^{\alpha \beta} \tau_{E}^{\alpha^{\prime} \beta^{\prime}} & +b_{\alpha \beta \alpha^{\prime} \beta^{\prime}}^{\text {hom }}\left(\tau_{E}^{\alpha \beta} \tau_{I}^{\alpha^{\prime} \beta^{\prime}}+\tau_{I}^{\alpha \beta} \tau_{E}^{\alpha^{\prime} \beta^{\prime}}\right) \\
& +c_{\alpha \beta \alpha^{\prime} \beta^{\prime}}^{\text {hom }} \tau_{I}^{\alpha \beta} \tau_{I}^{\alpha^{\prime} \beta^{\prime}} \geq C\left(\tau_{E}^{\alpha \beta} \tau_{E}^{\alpha \beta}+\tau_{I}^{\alpha \beta} \tau_{I}^{\alpha \beta}\right) \quad \forall\left(\tau_{E}, \tau_{I}\right) \in \mathbb{S}_{2} \times \mathbb{S}_{2} .
\end{aligned}
$$

Proof. We first note that with the variational formulations (8.1) we can calculate the homogenized coefficients as

$$
\begin{aligned}
a_{\alpha \beta \alpha^{\prime} \beta^{\prime}}^{\text {hom }} & =\frac{1}{\left|Y^{*}\right|} \int_{Y^{*}} a_{i j k l}(y)\left[\mathbf{M}_{i j}^{\alpha \beta}+\mathcal{E}_{i j, y}\left(\widetilde{\chi_{E}^{\alpha \beta}}\right)\right]\left[\mathbf{M}_{k l}^{\alpha^{\prime} \beta^{\prime}}+\mathcal{E}_{k l, y}\left(\widetilde{\chi_{E}^{\alpha^{\prime} \beta^{\prime}}}\right)\right] \mathrm{d} y, \\
b_{\alpha \beta \alpha^{\prime} \beta^{\prime}}^{\text {hom }} & =\frac{1}{\left|Y^{*}\right|} \int_{Y^{*}} a_{i j k l}(y)\left[y_{3} \mathbf{M}_{i j}^{\alpha \beta}+\mathcal{E}_{i j, y}\left(\widetilde{\chi_{I}^{\alpha \beta}}\right)\right]\left[\mathbf{M}_{k l}^{\alpha^{\prime} \beta^{\prime}}+\mathcal{E}_{k l, y}\left(\widetilde{\chi_{E}^{\alpha^{\prime} \beta^{\prime}}}\right)\right] \mathrm{d} y \\
& =\frac{1}{\left|Y^{*}\right|} \int_{Y^{*}} a_{i j k l}(y)\left[\mathbf{M}_{i j}^{\alpha \beta}+\mathcal{E}_{i j, y}\left(\widetilde{\chi_{E}^{\alpha \beta}}\right)\right]\left[y_{3} \mathbf{M}_{k l}^{\alpha^{\prime} \beta^{\prime}}+\mathcal{E}_{k l, y}\left(\widetilde{\chi_{I}^{\alpha^{\prime} \beta^{\prime}}}\right)\right] \mathrm{d} y, \\
c_{\alpha \beta \alpha^{\prime} \beta^{\prime}}^{\text {hom }} & =\frac{1}{\left|Y^{*}\right|} \int_{Y^{*}} a_{i j k l}(y)\left[y_{3} \mathbf{M}_{i j}^{\alpha \beta}+\mathcal{E}_{i j, y}\left(\widetilde{\chi_{I}^{\alpha \beta}}\right)\right]\left[y_{3} \mathbf{M}_{k l}^{\alpha^{\prime} \beta^{\prime}}+\mathcal{E}_{k l, y}\left(\widetilde{\chi_{I}^{\alpha^{\prime} \beta^{\prime}}}\right)\right] \mathrm{d} y .
\end{aligned}
$$

For every $\left(\tau_{E}, \tau_{I}\right) \in \mathbb{S}_{2} \times \mathbb{S}_{2}$, one has

$$
\begin{gathered}
a_{\alpha \beta \alpha^{\prime} \beta^{\prime}} \tau_{E}^{\alpha \beta} \tau_{E}^{\alpha^{\prime} \beta^{\prime}}+b_{\alpha \beta \alpha^{\prime} \beta^{\prime}}^{\mathrm{hom}}\left(\tau_{E}^{\alpha \beta} \tau_{I}^{\alpha^{\prime} \beta^{\prime}}+\tau_{I}^{\alpha \beta} \tau_{E}^{\alpha^{\prime} \beta^{\prime}}\right)+c_{\alpha \beta \alpha^{\prime} \beta^{\prime}}^{\text {hom }} \tau_{I}^{\alpha \beta} \tau_{I}^{\alpha^{\prime} \beta^{\prime}} \\
=\frac{1}{\left|Y^{*}\right|} \int_{Y^{*}} a_{i j k l}\left[M_{i j}+\mathcal{E}_{i j, y}(\Psi)\right]\left[M_{k l}+\mathcal{E}_{k l, y}(\Psi)\right] \mathrm{d} y,
\end{gathered}
$$

with $M=\left(\tau_{E}^{\alpha \beta}+y_{3} \tau_{I}^{\alpha \beta}\right) \mathbf{M}^{\alpha \beta}$, and $\Psi=\widetilde{\tau_{E}^{\alpha \beta} \widetilde{\chi_{E}^{\alpha \beta}}}+\tau_{I}^{\alpha \beta} \widetilde{\chi_{I}^{\alpha \beta}}$. By the coercivity of $a_{i j k l}$, see (6.2), we obtain

$$
\int_{Y^{*}} a_{i j k l}(y)\left[M_{i j}+\mathcal{E}_{i j, y}(\Psi)\right]\left[M_{k l}+\mathcal{E}_{k l, y}(\Psi)\right] \mathrm{d} y \geq c_{0} \int_{Y^{*}}\left[M_{i j}+\mathcal{E}_{i j, y}(\Psi)\right]\left[M_{i j}+\mathcal{E}_{i j, y}(\Psi)\right] \mathrm{d} y .
$$

Here we are again in the context of Lemma 7.2. This then yields with the equivalence of the norms that

$$
\begin{aligned}
\int_{Y^{*}}\left[M_{i j}+\mathcal{E}_{i j, y}(\Psi)\right]\left[M_{i j}+\mathcal{E}_{i j, y}(\Psi)\right] \mathrm{d} y & \geq C\left(\left|\tau_{E}\right|^{2}+\left|\tau_{I}\right|^{2}+\|\Psi\|_{L^{2}\left(Y^{*}\right)}^{2}\right) \\
& \geq C\left(\tau_{E}^{\alpha \beta} \tau_{E}^{\alpha \beta}+\tau_{I}^{\alpha \beta} \tau_{I}^{\alpha \beta}\right) \quad \forall\left(\tau_{E}, \tau_{I}\right) \in \mathbb{S}_{2} \times \mathbb{S}_{2} .
\end{aligned}
$$

\section{DifFEREnt BOUNDARY CONDITION}

In this section we want to emphasize on a change of the boundary condition, such that the previously free part is clamped, i.e. $\Gamma_{0}=\phi([0, \pi] \times\{0\} \cup[0, \pi] \times\{l\})$. We may note, that all presented estimates and resulting limits are not affected by the change of boundary conditions until we consider the split of $\mathcal{U}=\mathcal{U}_{I}+\mathcal{U}_{E}$. As in (5.1), we first obtain that $\mathcal{U}_{I}$ can be presented as

$$
\mathcal{U}_{2}\left(s_{1}, s_{2}\right)=U_{2}\left(s_{1}\right), \mathcal{U}_{1}\left(s_{1}, s_{2}\right)=-s_{2}^{c} U_{2}^{\prime}\left(s_{1}\right)+U_{1}\left(s_{1}\right), \mathcal{U}_{3}\left(s_{1}, s_{2}\right)=a\left(s_{2}^{c} U_{2}^{\prime \prime}\left(s_{1}\right)-U_{1}^{\prime}\left(s_{1}\right)\right),
$$

$U_{1} \in H^{1}(0, a \pi), U_{2} \in H^{2}(0, a \pi)$. With respect to our new boundary conditions we need that $\mathcal{U}_{2}\left(s_{1}, 0\right)=$ $\mathcal{U}_{2}\left(s_{1}, l\right)=0, \quad$ for a.e. $s_{1}$. Hence, we have $U_{2}\left(s_{1}\right)=0$ for a.e. $s_{1} \in(0, a \pi)$. With the same reasoning we get $U_{1}\left(s_{1}\right)=0$ for a.e. $s_{1} \in(0, a \pi)$. Therefore $D_{I}=\mathbb{D}_{I}=\{0\}$. 
Remark 9.1. In the applied forces we consider $F$ such that

$$
F_{1} \in L^{2}\left(0, l ; H_{0}^{1}(0, a \pi)\right) \cap H^{1}(\omega), \quad F_{2} \in H^{1}(\omega), \quad F_{3} \in L^{2}\left(0, l ; H_{0}^{2}(0, a \pi)\right) \cap H^{1}(\omega) .
$$

In the case of a fully clamped shell along $\partial \omega$ the assumptions on the forces do not change and we obtain $D_{I}=\mathbb{D}_{I}=0$. Hence, we immediately get equation (9.2).

Lemma 9.2. For every $\mathcal{U}$ in $\mathbb{D}_{E}$, where $\Gamma_{0}$ is given above, one has

$$
\left\|\mathcal{U}_{2}\right\|_{H^{1}\left(0, l ; L^{2}(0, a \pi)\right)}+\left\|\mathcal{U}_{1}\right\|_{H^{1}\left(0, l ;\left(H^{1}(0, a \pi)\right)^{\prime}\right)}+\left\|\mathcal{U}_{3}\right\|_{L^{2}\left(0, l ;\left(H^{2}(0, a \pi)\right)^{\prime}\right)} \leq C\|\mathcal{U}\|_{E} .
$$

Proof. This estimate is an immediate consequence of the fact that $D_{E}=H_{0}^{1}(\omega) \times H_{0}^{1}(\omega) \times L^{2}(\omega)$ and Lemma 5.2.

If we consider the linear elasticity problem presented in Section 6 and getting to the limit, as presented earlier, we obtain that the limit homogenized equation is given by

$$
\int_{\omega} a_{\alpha \beta \alpha^{\prime} \beta^{\prime}}^{\text {hom }} e_{\alpha \beta}\left(\mathcal{U}_{E}\right) e_{\alpha^{\prime} \beta^{\prime}}\left(\mathcal{V}_{E}\right) \mathrm{d} s^{\prime}=\frac{\mid Y^{\prime} *}{\left|Y^{\prime}\right|}\left\langle F, \mathcal{V}_{E}\right\rangle, \quad \forall \mathcal{V}_{E} \in \mathbb{D}_{E} .
$$

Now, we show that $\left\langle F, \mathcal{V}_{E}\right\rangle$ can be expressed in terms of $e_{\alpha^{\prime} \beta^{\prime}}\left(\mathcal{V}_{E}\right)$ for every $V \in \mathbb{D}_{E}$.

Denote $\mathcal{F}$ and $\widetilde{\mathcal{F}}$ the fields defined by

$$
\mathcal{F}(\cdot, 0)=0, \quad \frac{\partial \mathcal{F}}{\partial s_{2}}=F, \quad \widetilde{\mathcal{F}}(\cdot, 0)=0, \quad \frac{\partial \widetilde{\mathcal{F}}}{\partial s_{2}}=\mathcal{F} .
$$

Recall that the components of $F$ are given by (9.1).

Lemma 9.3. For every $V \in \mathbb{D}_{E}$ one has

$$
\left\langle F, \mathcal{V}_{E}\right\rangle=\int_{\omega}\left(\boldsymbol{F}_{11} e_{11}(V)+\boldsymbol{F}_{12} e_{12}(V)+\boldsymbol{F}_{22} e_{22}(V)\right) \mathrm{d} s_{1} \mathrm{~d} s_{2}
$$

where $\boldsymbol{F}_{11}=a F_{3}, \quad \boldsymbol{F}_{12}=-2\left(\mathcal{F}_{1}+a \partial_{1} \mathcal{F}_{3}\right), \quad \boldsymbol{F}_{22}=-\mathcal{F}_{2}+\partial_{1} \widetilde{\mathcal{F}}_{1}+a \partial_{11} \widetilde{\mathcal{F}}_{3}$.

Proof. Consider $V \in D_{E}$. One has

$$
\begin{aligned}
\int_{\omega} F_{3} V_{3} \mathrm{~d} s_{1} \mathrm{~d} s_{2} & =a \int_{\omega} F_{3} e_{11}(V) \mathrm{d} s_{1} \mathrm{~d} s_{2}-a \int_{\omega} F_{3} \partial_{1} V_{1} \mathrm{~d} s_{1} \mathrm{~d} s_{2}, \\
& =a \int_{\omega} F_{3} e_{11}(V) \mathrm{d} s_{1} \mathrm{~d} s_{2}+a \int_{\omega} \partial_{1} F_{3} V_{1} \mathrm{~d} s_{1} \mathrm{~d} s_{2} .
\end{aligned}
$$

Then

$$
\begin{aligned}
\int_{\omega}\left(F_{1}+a \partial_{1} F_{3}\right) V_{1} \mathrm{~d} s_{1} \mathrm{~d} s_{2} & =-\int_{\omega}\left(\mathcal{F}_{1}+a \partial_{1} \mathcal{F}_{3}\right) \partial_{2} V_{1} \mathrm{~d} s_{1} \mathrm{~d} s_{2} \\
& =-2 \int_{\omega}\left(\mathcal{F}_{1}+a \partial_{1} \mathcal{F}_{3}\right) e_{12}(V) \mathrm{d} s_{1} \mathrm{~d} s_{2}+\int_{\omega}\left(\mathcal{F}_{1}+a \partial_{1} \mathcal{F}_{3}\right) \partial_{1} V_{2} \mathrm{~d} s_{1} \mathrm{~d} s_{2} \\
& =-2 \int_{\omega}\left(\mathcal{F}_{1}+a \partial_{1} \mathcal{F}_{3}\right) e_{12}(V) \mathrm{d} s_{1} \mathrm{~d} s_{2}-\int_{\omega}\left(\partial_{1} \mathcal{F}_{1}+a \partial_{11} \mathcal{F}_{3}\right) V_{2} \mathrm{~d} s_{1} \mathrm{~d} s_{2}
\end{aligned}
$$

and finally

$$
\int_{\omega}\left(F_{2}-\partial_{1} \mathcal{F}_{1}-a \partial_{11} \mathcal{F}_{3}\right) V_{2} \mathrm{~d} s_{1} \mathrm{~d} s_{2}=-\int_{\omega}\left(\mathcal{F}_{2}-\partial_{1} \widetilde{\mathcal{F}}_{1}-a \partial_{11} \widetilde{\mathcal{F}}_{3}\right) \partial_{2} V_{2} \mathrm{~d} s_{1} \mathrm{~d} s_{2} .
$$




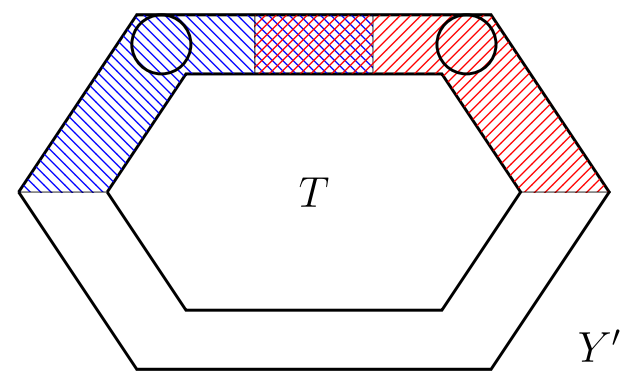

Figure A.1. Cell $Y^{\prime}$ and the perforated domain $Y^{\prime *}$.

With those calculations we obtain for every $V$ in $D_{E}$

$$
\begin{aligned}
\int_{\omega} F \cdot V \mathrm{~d} s_{1} \mathrm{~d} s_{2} & =\int_{\omega}\left(F_{1} V_{1}+F_{2} V_{2}+F_{3} V_{3}\right) \mathrm{d} s_{1} \mathrm{~d} s_{2} \\
& =\int_{\omega}\left(\left(F_{1}+a \partial_{1} F_{3}\right) V_{1}+F_{2} V_{2}+a F_{3} e_{11}(V)\right) \mathrm{d} s_{1} \mathrm{~d} s_{2} \\
& =\int_{\omega}\left(-2\left(\mathcal{F}_{1}+a \partial_{1} \mathcal{F}_{3}\right) e_{12}(V)+\left(F_{2}-\partial_{1} \mathcal{F}_{1}-a \partial_{11} \mathcal{F}_{3}\right) V_{2}+a F_{3} e_{11}(V)\right) \mathrm{d} s_{1} \mathrm{~d} s_{2} \\
& =\int_{\omega}\left(-2\left(\mathcal{F}_{1}+a \partial_{1} \mathcal{F}_{3}\right) e_{12}(V)+\left(-\mathcal{F}_{2}+\partial_{1} \widetilde{\mathcal{F}}_{1}+a \partial_{11} \widetilde{\mathcal{F}}_{3}\right) e_{22}(V)+a F_{3} e_{11}(V)\right) \mathrm{d} s_{1} \mathrm{~d} s_{2} .
\end{aligned}
$$

We conclude the proof by the density of $D_{E}$ in $\mathbb{D}_{E}$.

\section{Appendix A.}

\section{A.1. Proof of Proposition 1.1}

There exists $\kappa_{0}>0$ such that

$$
\mathcal{O}_{\kappa_{0}}^{\prime}=\left\{s \in \mathbb{R}^{2} \backslash \bar{T} \mid \operatorname{dist}(s, T)<\kappa_{0}\right\} \subset Y^{\prime *} .
$$

Since the boundary of $T$ is Lipschitz, there exist $R^{\prime}, R_{1}^{\prime}>0$ and $N \geq 2$ open sets $\mathcal{O}_{1}^{\prime}, \ldots, \mathcal{O}_{N}^{\prime}$ such that

- $\mathcal{O}_{i}^{\prime}$ is included in a ball of radius $R^{\prime}$ and is star-shaped with respect to a ball of radius $R_{1}^{\prime}, i \in\{1, \ldots, N\}$,

- $\mathcal{O}_{i}^{\prime} \cap \mathcal{O}_{i+1}^{\prime} \neq \emptyset, i \in\{1, \ldots, N-1\}$, and $\mathcal{O}_{N}^{\prime} \cap \mathcal{O}_{1}^{\prime} \neq \emptyset$,

- $\mathcal{O}_{\kappa_{0}}^{\prime} \subset \bigcup_{i=1}^{N} \mathcal{O}_{i}^{\prime} \subset Y^{\prime *}$.

Set $\mathcal{O}_{\kappa_{0}}=\mathcal{O}_{\kappa_{0}}^{\prime} \times(-\kappa, \kappa), \quad \mathcal{O}_{i}=\mathcal{O}_{i}^{\prime} \times(-\kappa, \kappa), \quad i \in\{1, \ldots, N\}$. One has

- $\mathbf{P}_{1}: \mathcal{O}_{i}$ is included in a ball of radius $R=R^{\prime}+\kappa$ and is star-shaped with respect to a ball of radius $R_{1}=\inf \left\{R_{1}^{\prime}, \kappa\right\}, i \in\{1, \ldots, N\}$,

- $\mathbf{P}_{2}: \mathcal{O}_{i} \cap \mathcal{O}_{i+1} \neq \emptyset, i \in\{1, \ldots, N-1\}$, and $\mathcal{O}_{N} \cap \mathcal{O}_{1} \neq \emptyset$,

- $\mathbf{P}_{3}: \mathcal{O}_{\kappa_{0}} \subset \bigcup_{i=1}^{N} \mathcal{O}_{i} \subset Y^{*}$.

Set $\mathcal{O}_{\kappa_{0}}^{\diamond}=\mathcal{O}_{\kappa_{0}} \cup(\bar{T} \times(-\kappa, \kappa))$. Below, we will use the classical extension result (Fig. A.1).

Lemma A.1. There exists an extension operator $\mathcal{P}$ from $H^{1}\left(\mathcal{O}_{\kappa_{0}}\right)$ into $H^{1}\left(\mathcal{O}_{\kappa_{0}}^{\diamond}\right)$ satisfying for all $\phi \in H^{1}\left(\mathcal{O}_{\kappa_{0}}\right)$

$$
\mathcal{P}(\phi)_{\mid \mathcal{O}_{\kappa_{0}}}=\phi, \quad\|\nabla(\mathcal{P}(\phi))\|_{L^{2}\left(\mathcal{O}_{\kappa_{0}}^{\diamond}\right)} \leq C\|\nabla \phi\|_{L^{2}\left(\mathcal{O}_{\kappa_{0}}\right)} .
$$

The constant only depends on $\partial T^{5}$.

\footnotetext{
${ }^{5}$ Note that if we transform the domain $\mathcal{O}_{\kappa_{0}}$ by a dilation, the constant does not change.
} 
Proof of Proposition 1.1. For every $\xi \in \Xi_{\varepsilon}$ and $\mathcal{O}_{i}, i \in\{1, \ldots, N\}$, if $\varepsilon$ s small enough, the domain $\Phi\left(\varepsilon \xi+\varepsilon \mathcal{O}_{i}\right)$ is included in a ball of radius $2 R \varepsilon$ and is star-shaped with respect to a ball of radius $R_{1} \varepsilon / 4$ (due to property $\mathbf{P}_{1}$ and Lemma A2 in [14]).

Now, let $u$ be a displacement belonging to $H^{1}\left(\mathcal{Q}_{\varepsilon}\right)^{3}$. For every $(\xi, i) \in \Xi_{\varepsilon} \times\{1, \ldots, N\}$ there exists a rigid displacement $r_{\xi, i}$ such that

$$
\left\|u-r_{\xi, i}\right\|_{L^{2}\left(\Phi\left(\varepsilon \xi+\varepsilon \mathcal{O}_{i}\right)\right)}+\varepsilon\left\|\nabla_{x}\left(u-r_{\xi, i}\right)\right\|_{L^{2}\left(\Phi\left(\varepsilon \xi+\varepsilon \mathcal{O}_{i}\right)\right)} \leq C \varepsilon\|e(u)\|_{L^{2}\left(\Phi\left(\varepsilon \xi+\varepsilon \mathcal{O}_{i}\right)\right)} .
$$

The constant does not depend on $\varepsilon, \xi$ and $\mathcal{O}_{i}$, it only depends on the ratio $R / R_{1}$ (see [14], Thm. 2.3). Then, step by step we compare the rigid displacements $r_{\xi, 1}, r_{\xi, 2}, \ldots, r_{\xi, N}$ thanks to the properties $\mathbf{P}_{2}$ and $\mathbf{P}_{3}$. To do that, observe that there exist two constants independent of $\varepsilon$ and $\xi$ such that

$$
\begin{aligned}
c \varepsilon^{3}\left|\mathcal{O}_{i} \cap \mathcal{O}_{i+1}\right| & \leq\left|\Phi\left(\varepsilon \xi+\varepsilon \mathcal{O}_{i} \cap \mathcal{O}_{i+1}\right)\right| \leq C \varepsilon^{3}\left|\mathcal{O}_{i} \cap \mathcal{O}_{i+1}\right|, \quad i \in\{1, \ldots, N-1\} \\
c \varepsilon^{3}\left|\mathcal{O}_{N} \cap \mathcal{O}_{1}\right| & \leq\left|\Phi\left(\varepsilon \xi+\varepsilon \mathcal{O}_{N} \cap \mathcal{O}_{1}\right)\right| \leq C \varepsilon^{3}\left|\mathcal{O}_{N} \cap \mathcal{O}_{1}\right|
\end{aligned}
$$

As a consequence, there exists a rigid displacement $r_{\xi}$ such that

$$
\left\|u-r_{\xi}\right\|_{L^{2}\left(\Phi\left(\varepsilon \xi+\varepsilon Y^{*}\right)\right)}+\varepsilon\left\|\nabla_{x}\left(u-r_{\xi}\right)\right\|_{L^{2}\left(\Phi\left(\varepsilon \xi+\varepsilon Y^{*}\right)\right)} \leq C \varepsilon\|e(u)\|_{L^{2}\left(\Phi\left(\varepsilon \xi+\varepsilon Y^{*}\right)\right)} .
$$

The constant does not depend on $\varepsilon$ and $\xi$. Then, taking mean values, one can replace $r_{\xi}$ by $\mathbf{r}_{\xi}$ with the same estimate

$$
\mathbf{r}_{\xi}(x)=\mathcal{M}_{\Phi\left(\varepsilon \xi+\varepsilon Y^{*}\right)}(u)+\mathcal{M}_{\Phi\left(\varepsilon \xi+\varepsilon Y^{*}\right)}\left(\nabla u-(\nabla u)^{T}\right)\left(x-G_{\xi}\right)
$$

where $\mathcal{M}_{\Phi\left(\varepsilon \xi+\varepsilon Y^{*}\right)}(\phi)$ is the mean value of $\phi \in L^{1}\left(\Phi\left(\varepsilon \xi+\varepsilon Y^{*}\right)\right)$ in the open set $\Phi\left(\varepsilon \xi+\varepsilon Y^{*}\right)$ and $G_{\xi}$ is its center of mass.

At this point, transform the domain $\Phi\left(\varepsilon \xi+\varepsilon Y^{*}\right)$ by the inverse map $z \in Y^{*} \longmapsto \Phi(\varepsilon \xi+\varepsilon z)$, then apply Lemma A.1 in order to extend the function in the hole $T$ and finally transform by the map $z \in Y \longmapsto \Phi(\varepsilon \xi+\varepsilon z)$ and to the result add the displacement $r_{\xi}$. The $L^{2}$ norm of the strain tensor of the extended displacement (now defined in $\Phi(\varepsilon \xi+\varepsilon Y)$ ) is bounded by a constant (independent of $\varepsilon$ and $\xi$ ) multiply by $\|e(u)\|_{L^{2}\left(\Phi\left(\varepsilon \xi+\varepsilon Y^{*}\right)\right)}$.

We apply this process to every domain of $\varepsilon \xi+\varepsilon Y^{*}, \xi \in \Xi_{\varepsilon}$. Finally, we obtain an extension of the displacement $u$ satisfying (1.6).

\section{A.2. Two lemmas}

For the definitions and properties of the unfolding operators $\mathcal{T}_{\varepsilon}, \mathcal{M}_{\varepsilon}$ we refer to [9,10] and Lemma A.2 is proved in [10]. Let $\Omega$ be a bounded domain in $\mathcal{R}^{N}$ with Lipschitz boundary and $Y=\Pi_{i=1}^{N}\left(0, l_{i}\right), l_{i}>0$, $i=1, \ldots, N$.

Lemma A.2. Suppose $p \in(1,+\infty)$. Let $\left\{\left(\boldsymbol{u}_{\varepsilon, \delta}, \boldsymbol{v}_{\varepsilon, \delta}\right)\right\}_{\varepsilon, \delta}$ be a sequence in $W^{1, p}(\Omega)^{N} \times W^{1, p}(\Omega)^{N \times N}$ (with $\boldsymbol{v}_{\varepsilon, \delta}$ a symmetric matrix) converging weakly to $(\boldsymbol{u}, \boldsymbol{v})$ in $W^{1, p}(\Omega)^{N} \times W^{1, p}(\Omega)^{N \times N}$.

Assume furthermore that there exist $\mathcal{X}$ in $L^{p}(\Omega)^{N \times N}$ and $\widehat{v}$ in $L^{p}\left(\Omega ; W_{\text {per, } 0}^{1, p}(Y)\right)^{N \times N}$ such that as $(\varepsilon, \delta) \rightarrow$ $(0,0)$

$$
\begin{aligned}
\frac{1}{\delta}\left(e\left(\boldsymbol{u}_{\varepsilon, \delta}\right)+\boldsymbol{v}_{\varepsilon, \delta}\right) & \rightarrow \mathcal{X} \quad \text { weakly } \text { in } L^{p}(\Omega)^{N \times N}, \\
\mathcal{T}_{\varepsilon, \delta}\left(\nabla \boldsymbol{v}_{\varepsilon, \delta}\right) & \rightarrow \nabla \boldsymbol{v}+\nabla_{y} \widehat{\boldsymbol{v}} \quad \text { weakly } \quad \text { in } L^{p}(\Omega \times Y)^{N \times N \times N} .
\end{aligned}
$$

Then $\boldsymbol{u}$ belongs to $W^{2, p}(\Omega)^{N}$ and there exists $\mathfrak{u} \in L^{p}\left(\Omega ; W_{\text {per }, 0}^{1, p}(Y)\right)^{N}$ such that, up to a subsequence,

$$
\begin{array}{lll}
\text { if } \quad \frac{\varepsilon}{\delta} \rightarrow \theta \in[0,+\infty), & \frac{1}{\delta} \mathcal{T}_{\varepsilon, \delta}\left(e\left(\boldsymbol{u}_{\varepsilon, \delta}\right)+\boldsymbol{v}_{\varepsilon, \delta}\right) \rightarrow \mathcal{X}+e_{y}(\mathfrak{u})+\theta \widehat{\boldsymbol{v}} \quad \text { weakly } \quad \text { in } \quad L^{p}(\Omega \times Y)^{N \times N}, \\
\text { if } \quad \frac{\varepsilon}{\delta} \rightarrow+\infty, & \widehat{\boldsymbol{v}}=e_{y}(\mathfrak{u}) .
\end{array}
$$


Proof. First, from (A.3) one obtains that $e(\mathbf{u})+\mathbf{v}=0$, then since $\Omega$ is a bounded domain with Lipschitz boundary $\mathbf{u}$ belongs to $W^{2, p}(\Omega)^{N}$. We also deduce from this convergence and the Korn inequality that $\mathbf{u}_{\varepsilon, \delta}$ strongly converges to $\mathbf{u}$ in $W^{1, p}(\Omega)^{N}$.

Then, up to a subsequence, there exists $\widehat{\mathcal{X}} \in L^{p}(\Omega \times Y)^{N}$ such that

$$
\frac{1}{\delta} \mathcal{T}_{\varepsilon, \delta}\left(e\left(\mathbf{u}_{\varepsilon, \delta}\right)+\mathbf{v}_{\varepsilon, \delta}\right) \rightarrow \widehat{\mathcal{X}} \quad \text { weakly } \quad \text { in } \quad L^{p}(\Omega \times Y)^{N \times N} .
$$

Step 1. In this first step we assume that $\frac{\varepsilon}{\delta} \rightarrow \theta \in[0,+\infty)$.

Introduce the function $\mathbf{Z}_{\varepsilon, \delta}$ belonging to $L^{p}\left(\Omega ; W^{1, p}(Y)\right)^{N}$, defined as

$$
\mathbf{Z}_{\varepsilon, \delta}=\frac{1}{\varepsilon} \mathcal{T}_{\varepsilon}\left(\mathbf{u}_{\varepsilon, \delta}-\mathcal{M}_{\varepsilon}\left(\mathbf{u}_{\varepsilon, \delta}\right)\right)-\mathcal{M}_{\varepsilon}\left(\nabla \mathbf{u}_{\varepsilon, \delta}\right) \cdot y^{c} .
$$

Its gradient and symmetric gradient with respect to $y$ are

$$
\begin{aligned}
\nabla_{y} \mathbf{Z}_{\varepsilon, \delta} & =\mathcal{T}_{\varepsilon}\left(\nabla \mathbf{u}_{\varepsilon, \delta}\right)-\mathcal{M}_{\varepsilon}\left(\nabla \mathbf{u}_{\varepsilon, \delta}\right) \\
e_{y}\left(\mathbf{Z}_{\varepsilon, \delta}\right) & =\mathcal{T}_{\varepsilon}\left(e\left(\mathbf{u}_{\varepsilon, \delta}\right)\right)-\mathcal{M}_{\varepsilon}\left(e\left(\mathbf{u}_{\varepsilon, \delta}\right)\right) \\
& =\mathcal{T}_{\varepsilon}\left(e\left(\mathbf{u}_{\varepsilon, \delta}\right)+\mathbf{v}_{\varepsilon, \delta}\right)-\left(\mathcal{T}_{\varepsilon}\left(\mathbf{v}_{\varepsilon, \delta}\right)-\mathcal{M}_{\varepsilon}\left(\mathbf{v}_{\varepsilon, \delta}\right)\right)-\mathcal{M}_{\varepsilon}\left(e\left(\mathbf{u}_{\varepsilon, \delta}\right)+\mathbf{v}_{\varepsilon, \delta}\right)
\end{aligned}
$$

Convergence (A.3) $)_{1}$ on one side together with the fact that $\left\|\nabla \mathbf{v}_{\varepsilon, \delta}\right\|_{L^{p}(\Omega)}$ and $\frac{\varepsilon}{\delta}$ are bounded, give

$$
\left\|e_{y}\left(\mathbf{Z}_{\varepsilon, \delta}\right)\right\|_{L^{p}(\Omega \times Y)^{N}} \leq C(\delta+\varepsilon) \leq C \delta .
$$

The Korn inequality implies

$$
\left\|\mathbf{Z}_{\varepsilon, \delta}\right\|_{L^{p}\left(\Omega ; W^{1, p}(Y)\right)} \leq C \delta
$$

Consequently, up to a subsequence, there exists $\widehat{\mathbf{Z}}$ in $L^{p}\left(\Omega ; W^{1, p}(Y)\right)^{N}$ such that,

$$
\frac{1}{\delta} \mathbf{Z}_{\varepsilon, \delta} \rightarrow \widehat{\mathbf{Z}} \quad \text { weakly } \quad \text { in } \quad L^{p}\left(\Omega ; W^{1, p}(Y)\right)^{N} .
$$

By (A.6) one has

$$
\frac{1}{\delta} \mathcal{T}_{\varepsilon}\left(e\left(\mathbf{u}_{\varepsilon, \delta}\right)+\mathbf{v}_{\varepsilon, \delta}\right)=\frac{1}{\delta} e_{y}\left(\mathbf{Z}_{\varepsilon, \delta}\right)+\frac{\varepsilon}{\delta} \frac{\mathcal{T}_{\varepsilon}\left(\mathbf{v}_{\varepsilon, \delta}\right)-\mathcal{M}_{\varepsilon}\left(\mathbf{v}_{\varepsilon, \delta}\right)}{\varepsilon}+\frac{1}{\delta} \mathcal{M}_{\varepsilon}\left(e\left(\mathbf{u}_{\varepsilon, \delta}\right)+\mathbf{v}_{\varepsilon, \delta}\right) .
$$

Then going to the limit using (A.7) and Proposition 1.25 and Theorem 1.41 in [10]

$$
\frac{1}{\delta} \mathcal{T}_{\varepsilon}\left(\nabla \mathbf{u}_{\varepsilon, \delta}+\mathbf{v}_{\varepsilon, \delta}\right) \rightarrow \widehat{\mathcal{X}}=e_{y}(\widehat{\mathbf{Z}})+\theta\left(\nabla \mathbf{v} y^{c}+\widehat{\mathbf{v}}\right)+\mathcal{X} \quad \text { weakly } \quad \text { in } \quad L^{p}(\Omega \times Y)^{N \times N}
$$

Now, we prove that

$$
\mathfrak{u}=\widehat{\mathbf{Z}}-\frac{\theta}{2} \sum_{j, k=1}^{N} \frac{\partial^{2} \mathbf{u}}{\partial x_{j} \partial x_{k}}\left(y_{j}^{c} y_{k}^{c}-\mathcal{M}_{Y}\left(y_{j}^{c} y_{k}^{c}\right)\right)
$$

is periodic (note that this function belongs to $L^{p}\left(\Omega ; W^{1, p}(Y)\right)^{N}$ ).

We proceed as in the proof of Theorem 1.36 in [10], one first evaluates the difference of the traces of $\mathbf{Z}_{\varepsilon, \delta}$ on the faces $Y_{1}=\{0\} \times(0,1)^{N-1}$ and $Y_{1}+\mathbf{e}_{1}$. For a.e. $\left(x, y^{\prime}\right) \in \Omega \times Y_{1}$, one has

$$
\begin{aligned}
& \mathbf{Z}_{\varepsilon, \delta}\left(x, y^{\prime}+\mathbf{e}_{i}\right)-\mathbf{Z}_{\varepsilon, \delta}\left(x, y^{\prime}\right) \\
& \quad=\frac{1}{\varepsilon}\left(\mathcal{T}_{\varepsilon}\left(\mathbf{u}_{\varepsilon, \delta}\right)\left(x, y^{\prime}+\mathbf{e}_{1}\right)-\mathcal{T}_{\varepsilon}\left(\mathbf{u}_{\varepsilon, \delta}\right)\left(x, y^{\prime}\right)\right)-\mathcal{M}_{\varepsilon}\left(\frac{\partial \mathbf{u}_{\varepsilon, \delta}}{\partial x_{1}}\right)(x) \\
& \quad=\frac{1}{\varepsilon}\left(\mathcal{T}_{\varepsilon}\left(\mathbf{u}_{\varepsilon, \delta}\right)\left(x+\varepsilon \mathbf{e}_{1}, y^{\prime}\right)-\mathcal{T}_{\varepsilon}\left(\mathbf{u}_{\varepsilon, \delta}\right)\left(x, y^{\prime}\right)\right)-\mathcal{M}_{\varepsilon}\left(\frac{\partial \mathbf{u}_{\varepsilon, \delta}}{\partial x_{1}}\right)(x) .
\end{aligned}
$$


Let $\Phi$ be in $\mathcal{D}\left(\Omega \times Y_{1}\right)^{N}$, one has successively

$$
\begin{aligned}
\int_{\Omega \times Y_{1}} & \left(\mathbf{Z}_{\varepsilon, \delta}\left(x, y^{\prime}+\mathbf{e}_{i}\right)-\mathbf{Z}_{\varepsilon, \delta}\left(x, y^{\prime}\right)\right) \cdot \Phi\left(x, y^{\prime}\right) \mathrm{d} x \mathrm{~d} y^{\prime} \\
= & \int_{\Omega \times Y_{1}}\left[\frac{1}{\varepsilon}\left(\mathcal{T}_{\varepsilon}\left(\mathbf{u}_{\varepsilon, \delta}\right)\left(x+\varepsilon \mathbf{e}_{1}, y^{\prime}\right)-\mathcal{T}_{\varepsilon}\left(\mathbf{u}_{\varepsilon, \delta}\right)\left(x, y^{\prime}\right)\right)-\mathcal{M}_{\varepsilon}\left(\frac{\partial \mathbf{u}_{\varepsilon, \delta}}{\partial x_{1}}\right)(x)\right] \cdot \Phi\left(x, y^{\prime}\right) \mathrm{d} x \mathrm{~d} y^{\prime} \\
= & \int_{\Omega \times Y_{1}} \mathcal{T}_{\varepsilon}\left(\mathbf{u}_{\varepsilon, \delta}\right)\left(x, y^{\prime}\right) \cdot \frac{\Phi\left(x-\varepsilon \mathbf{e}_{1}, y^{\prime}\right)-\Phi\left(x, y^{\prime}\right)}{\varepsilon} \mathrm{d} x \mathrm{~d} y^{\prime}-\int_{\Omega \times Y_{1}} \mathcal{M}_{\varepsilon}\left(\frac{\partial \mathbf{u}_{\varepsilon, \delta}}{\partial x_{1}}\right)(x) \cdot \Phi\left(x, y^{\prime}\right) \mathrm{d} x \mathrm{~d} y^{\prime} \\
= & \int_{\Omega \times Y_{1}}\left(\mathbf{u}_{\varepsilon, \delta}(x)-\mathcal{T}_{\varepsilon}\left(\mathbf{u}_{\varepsilon, \delta}\right)\left(x, y^{\prime}\right)\right) \cdot \frac{\partial \Phi}{\partial x_{1}}\left(x, y^{\prime}\right) \mathrm{d} x \mathrm{~d} y^{\prime}+\int_{\Omega \times Y_{1}}\left(\frac{\partial \mathbf{u}_{\varepsilon, \delta}}{\partial x_{1}}-\mathcal{M}_{\varepsilon}\left(\frac{\partial \mathbf{u}_{\varepsilon, \delta}}{\partial x_{1}}\right)\right) \cdot \Phi\left(x, y^{\prime}\right) \mathrm{d} x \mathrm{~d} y^{\prime} \\
+ & \int_{\Omega \times Y_{1}} \mathcal{T}_{\varepsilon}\left(\mathbf{u}_{\varepsilon, \delta}\right)\left(x, y^{\prime}\right) \cdot \frac{\Phi\left(x-\varepsilon \mathbf{e}_{1}, y^{\prime}\right)-\Phi\left(x, y^{\prime}\right)+\varepsilon \mathbf{e}_{1} \cdot \nabla_{x} \Phi\left(x, y^{\prime}\right)}{\varepsilon} \mathrm{d} x \mathrm{~d} y^{\prime}
\end{aligned}
$$

then

$$
\begin{aligned}
& =\int_{\Omega \times Y_{1}}\left(\mathcal{M}_{\varepsilon}\left(\mathbf{u}_{\varepsilon, \delta}\right)(x)-\mathcal{T}_{\varepsilon}\left(\mathbf{u}_{\varepsilon, \delta}\right)\left(x, y^{\prime}\right)\right) \cdot \frac{\partial \Phi}{\partial x_{1}}\left(x, y^{\prime}\right) \mathrm{d} x \mathrm{~d} y^{\prime} \\
& +\int_{\Omega \times Y_{1}}\left(\mathbf{u}_{\varepsilon, \delta}(x)-\mathcal{M}_{\varepsilon}\left(\mathbf{u}_{\varepsilon, \delta}\right)(x) \cdot \frac{\partial \Phi}{\partial x_{1}}\left(x, y^{\prime}\right) \mathrm{d} x \mathrm{~d} y^{\prime}+\int_{\Omega \times Y_{1}}\left(\frac{\partial \mathbf{u}_{\varepsilon, \delta}}{\partial x_{1}}-\mathcal{M}_{\varepsilon}\left(\frac{\partial \mathbf{u}_{\varepsilon, \delta}}{\partial x_{1}}\right)\right) \cdot \Phi\left(x, y^{\prime}\right) \mathrm{d} x \mathrm{~d} y^{\prime}\right. \\
& +\int_{\Omega \times Y_{1}} \mathcal{T}_{\varepsilon}\left(\mathbf{u}_{\varepsilon, \delta}\right)\left(x, y^{\prime}\right) \cdot \frac{\Phi\left(x-\varepsilon \mathbf{e}_{1}, y^{\prime}\right)-\Phi\left(x, y^{\prime}\right)+\varepsilon \mathbf{e}_{1} \cdot \nabla_{x} \Phi\left(x, y^{\prime}\right)}{\varepsilon} \mathrm{d} x \mathrm{~d} y^{\prime} .
\end{aligned}
$$

The last right-hand side is equal to (see [10], Prop. 1.24)

$$
\begin{aligned}
\int_{\Omega \times Y_{1}} & \left(\mathcal{M}_{\varepsilon}\left(\mathbf{u}_{\varepsilon, \delta}\right)(x)-\mathcal{T}_{\varepsilon}\left(\mathbf{u}_{\varepsilon, \delta}\right)\left(x, y^{\prime}\right)\right) \cdot \frac{\partial \Phi}{\partial x_{1}}\left(x, y^{\prime}\right) \mathrm{d} x \mathrm{~d} y^{\prime} \\
+ & \int_{\Omega} \mathbf{u}_{\varepsilon, \delta}(x) \cdot\left(\int_{Y} \frac{\partial \Phi}{\partial x_{1}}\left(x, y^{\prime}\right) \mathrm{d} y^{\prime}\right)-\mathcal{M}_{\varepsilon}\left(\int_{Y} \frac{\partial \Phi}{\partial x_{1}}\left(x, y^{\prime}\right) \mathrm{d} y^{\prime}\right) \mathrm{d} x \\
& +\int_{\Omega} \frac{\partial \mathbf{u}_{\varepsilon, \delta}}{\partial x_{1}}(x) \cdot\left(\int_{Y} \Phi\left(x, y^{\prime}\right) \mathrm{d} y^{\prime}\right)-\mathcal{M}_{\varepsilon}\left(\int_{Y} \Phi\left(x, y^{\prime}\right) \mathrm{d} y^{\prime}\right) \mathrm{d} x \\
& +\int_{\Omega \times Y_{1}} \mathcal{T}_{\varepsilon}\left(\mathbf{u}_{\varepsilon, \delta}\right)\left(x, y^{\prime}\right) \cdot \frac{\Phi\left(x-\varepsilon \mathbf{e}_{1}, y^{\prime}\right)-\Phi\left(x, y^{\prime}\right)+\varepsilon \mathbf{e}_{1} \cdot \nabla_{x} \Phi\left(x, y^{\prime}\right)}{\varepsilon} \mathrm{d} x \mathrm{~d} y^{\prime} .
\end{aligned}
$$

Divide by $\delta$ and then pass to the limit using Propositions 1.38 and 1.39 [10]. It yields

$$
\begin{aligned}
\int_{\Omega \times Y_{1}} & \frac{\mathbf{Z}_{\varepsilon, \delta}\left(x, y^{\prime}+\mathbf{e}_{i}\right)-\mathbf{Z}_{\varepsilon, \delta}\left(x, y^{\prime}\right)}{\delta} \cdot \Phi(x, y) \mathrm{d} x \mathrm{~d} y^{\prime} \\
& \longrightarrow \int_{\Omega \times Y_{1}}-\theta\left(\nabla \mathbf{u}(x) y^{c}\right) \cdot \frac{\partial \Phi}{\partial x_{1}}\left(x, y^{\prime}\right) \mathrm{d} x \mathrm{~d} y^{\prime}+\frac{\theta}{2} \int_{\Omega \times Y_{1}} \mathbf{u}(x) \cdot \frac{\partial^{2} \Phi}{\partial x_{1}^{2}}\left(x, y^{\prime}\right) \mathrm{d} x \mathrm{~d} y^{\prime} \\
= & \int_{\Omega \times Y_{1}} \theta \sum_{k=2}^{N} \frac{\partial^{2} \mathbf{u}}{\partial x_{1} \partial x_{k}}(x) y_{k}^{\prime c} \cdot \Phi\left(x, y^{\prime}\right) \mathrm{d} x \mathrm{~d} y^{\prime} .
\end{aligned}
$$

Hence, for a.e. $\left(x, y^{\prime}\right) \in \Omega \times Y_{1}, \widehat{\mathbf{Z}}\left(x, y^{\prime}+\mathbf{e}_{i}\right)-\widehat{\mathbf{Z}}\left(x, y^{\prime}\right)=\theta \sum_{k=2}^{N} \frac{\partial^{2} \mathbf{u}}{\partial x_{1} \partial x_{k}}(x) y_{k}^{\prime c}$. We obtain similar equalities for the difference of the traces of $\widehat{\mathbf{Z}}$ over the other faces of $Y$. That proves the claim. Then, a straightforward calculation gives (using $\nabla e(\mathbf{u})+\nabla \mathbf{v}=0$ )

$$
e_{y}(\mathfrak{u})=e_{y}(\widehat{\mathbf{Z}})-\theta \sum_{k=1}^{N} \frac{\partial e(\mathbf{u})}{\partial x_{k}} y_{k}^{c}=e_{y}(\widehat{\mathbf{Z}})+\theta \sum_{k=1}^{N} \frac{\partial \mathbf{v}}{\partial x_{k}} y_{k}^{c} .
$$


With (A.8), that gives the convergence (A.4) $)_{1}$.

Step 2. In this step we assume that $\frac{\varepsilon}{\delta} \rightarrow+\infty$.

Again we consider the function $\mathbf{Z}_{\varepsilon, \delta}$ introduced in (A.5). Now, it satisfies

$$
\left\|\mathbf{Z}_{\varepsilon, \delta}\right\|_{L^{p}\left(\Omega ; W^{1, p}(Y)\right)} \leq C \varepsilon
$$

Hence, up to a subsequence, there exists $\widehat{\mathbf{Z}}$ in $L^{p}\left(\Omega ; W^{1, p}(Y)\right)^{N}$ such that,

$$
\frac{1}{\varepsilon} \mathbf{Z}_{\varepsilon, \delta} \rightarrow \widehat{\mathbf{Z}} \quad \text { weakly } \quad \text { in } \quad L^{p}\left(\Omega ; W^{1, p}(Y)\right)^{N} .
$$

Observe that

$$
\begin{aligned}
\frac{1}{\varepsilon} \mathcal{T}_{\varepsilon}\left(e\left(\mathbf{u}_{\varepsilon, \delta}\right)+\mathbf{v}_{\varepsilon, \delta}\right) & =\frac{\delta}{\varepsilon} \frac{1}{\delta} \mathcal{T}_{\varepsilon}\left(e\left(\mathbf{u}_{\varepsilon, \delta}\right)+\mathbf{v}_{\varepsilon, \delta}\right) \longrightarrow 0 \quad \text { strongly } \quad \text { in } \quad L^{p}(\Omega \times Y)^{N \times N}, \\
\frac{1}{\varepsilon} \mathcal{M}_{\varepsilon}\left(e\left(\mathbf{u}_{\varepsilon, \delta}\right)+\mathbf{v}_{\varepsilon, \delta}\right) & =\frac{\delta}{\varepsilon} \frac{1}{\delta} \mathcal{M}_{\varepsilon}\left(e\left(\mathbf{u}_{\varepsilon, \delta}\right)+\mathbf{v}_{\varepsilon, \delta}\right) \longrightarrow 0 \quad \text { strongly } \quad \text { in } \quad L^{p}(\Omega)^{N \times N}
\end{aligned}
$$

One has

$$
\frac{1}{\varepsilon} \mathcal{T}_{\varepsilon}\left(e\left(\mathbf{u}_{\varepsilon, \delta}\right)+\mathbf{v}_{\varepsilon, \delta}\right)=\frac{1}{\varepsilon} e_{y}\left(\mathbf{Z}_{\varepsilon, \delta}\right)+\frac{\mathcal{T}_{\varepsilon}\left(\mathbf{v}_{\varepsilon, \delta}\right)-\mathcal{M}_{\varepsilon}\left(\mathbf{v}_{\varepsilon, \delta}\right)}{\varepsilon}+\frac{1}{\varepsilon} \mathcal{M}_{\varepsilon}\left(e\left(\mathbf{u}_{\varepsilon, \delta}\right)+\mathbf{v}_{\varepsilon, \delta}\right) .
$$

Passing to the limit in the above equality gives

$$
e_{y}(\widehat{\mathbf{Z}})+\nabla \mathbf{v} y^{c}+\widehat{\mathbf{v}}=0
$$

Then, as in the previous step we prove that

$$
\mathfrak{v}=\widehat{\mathbf{Z}}-\frac{1}{2} \sum_{j, k=1}^{N} \frac{\partial^{2} \mathbf{u}}{\partial x_{j} \partial x_{k}}\left(y_{j}^{c} y_{k}^{c}-\mathcal{M}_{Y}\left(y_{j}^{c} y_{k}^{c}\right)\right)
$$

is periodic. Thus $(\text { A.4 })_{2}$ is proved with $\mathfrak{u}=-\mathfrak{v}$.

As a consequence of Lemma A.2 one has (see also [10], Lem. 11.11).

Lemma A.3. Suppose $p \in(1,+\infty)$. Let $\left\{\left(u_{\varepsilon, \delta}, v_{\varepsilon, \delta}\right)\right\}_{\varepsilon, \delta}$ be a sequence in $W^{1, p}(\Omega) \times W^{1, p}(\Omega)^{N}$ converging weakly to $(u, v)$ in $W^{1, p}(\Omega) \times W^{1, p}(\Omega)^{N}$. Assume furthermore that there exist $\mathcal{X}$ in $L^{p}(\Omega)^{N}$ and $\widehat{v}$ in $L^{p}\left(\Omega ; W_{\text {per }, 0}^{1, p}(Y)\right)^{N}$ such that as $(\varepsilon, \delta) \rightarrow(0,0)$

$$
\begin{aligned}
\frac{1}{\delta}\left(\nabla u_{\varepsilon, \delta}+v_{\varepsilon, \delta}\right) & \rightarrow \mathcal{X} \quad \text { weakly } \quad \text { in } L^{p}(\Omega)^{N}, \\
\mathcal{T}_{\varepsilon, \delta}\left(\nabla v_{\varepsilon, \delta}\right) & \rightarrow \nabla v+\nabla_{y} \widehat{v} \quad \text { weakly in } L^{p}(\Omega \times Y)^{N \times N} .
\end{aligned}
$$

Then $u$ belongs to $W^{2, p}(\Omega)$ and there exists $\mathfrak{u} \in L^{p}\left(\Omega ; W_{\text {per }, 0}^{1, p}(Y)\right)$ such that, up to a subsequence,

$$
\begin{array}{ll}
\text { if } \quad \frac{\varepsilon}{\delta} \rightarrow \theta \in[0,+\infty), & \frac{1}{\delta} \mathcal{T}_{\varepsilon, \delta}\left(\nabla u_{\varepsilon, \delta}+v_{\varepsilon, \delta}\right) \rightarrow \mathcal{X}+\nabla_{y} \mathfrak{u}+\theta \widehat{v} \quad \text { weakly } \quad \text { in } \quad L^{p}(\Omega \times Y)^{N}, \\
\text { if } \quad \frac{\varepsilon}{\delta} \rightarrow+\infty, & \widehat{v}=\nabla_{y} \mathfrak{u} .
\end{array}
$$

Proof. Consider the field $\mathbf{u}_{\varepsilon, \delta} \in W^{1, p}(\Omega)^{N}$ and the symmetric matrix field $\mathbf{v}_{\varepsilon, \delta} \in W^{1, p}(\Omega)^{N \times N}$ defined by

$$
\begin{array}{rlrl}
\mathbf{u}_{\varepsilon, \delta} & =\left(u_{\varepsilon, \delta}, 0, \ldots, 0\right), & \left(\mathbf{v}_{\varepsilon, \delta}\right)_{11}=v_{1, \varepsilon}, \\
\left(\mathbf{v}_{\varepsilon, \delta}\right)_{1 i} & =\left(\mathbf{v}_{\varepsilon, \delta}\right)_{i 1}=\frac{1}{2} v_{i, \varepsilon}, \quad\left(\mathbf{v}_{\varepsilon, \delta}\right)_{i j}=0 \text { if }(i, j) \in\{2, \ldots, N\}^{2} .
\end{array}
$$

These fields satisfy the assumptions of Lemma A.2 and the convergences (A.3). Therefore, the results in (A.4) give (A.10). 


\section{A.3. Postponed proofs}

Proof of Proposition 5.1. Step 1. We start by showing the norm equivalences.

Take $\mathcal{V} \in D_{I}\left(\right.$ resp. $\left.\mathbb{D}_{I}\right)$, one has

$$
e_{11}(\mathcal{V})=-\frac{1}{a} \mathcal{V}_{3}, \quad e_{12}(\mathcal{V})=e_{22}(\mathcal{V})=0
$$

Now, the $2 D$-Korn inequality gives (recall that $\mathcal{V}_{1}=\mathcal{V}_{2}=0$ on $\Gamma_{0}$ )

$$
\left\|\mathcal{V}_{1}\right\|_{H^{1}(\omega)}^{2}+\left\|\mathcal{V}_{2}\right\|_{H^{1}(\omega)}^{2} \leq C\left\|\mathcal{V}_{3}\right\|_{L^{2}(\omega)}^{2} .
$$

Then we obtain

$$
\|\mathcal{V}\|_{\left[H^{1}(\omega)\right]^{2} \times L^{2}(\omega)}^{2}=\left\|\mathcal{V}_{1}\right\|_{H^{1}(\omega)}^{2}+\left\|\mathcal{V}_{2}\right\|_{H^{1}(\omega)}^{2}+\left\|\mathcal{V}_{3}\right\|_{L^{2}(\omega)}^{2} \leq C\left\|\mathcal{V}_{3}\right\|_{L^{2}(\omega)}^{2}=C\|\mathcal{V}\|_{D_{I}}^{2} .
$$

On the contrary, to estimate $\|\cdot\|_{D_{I}}$ by $\|\cdot\|_{\left[H^{1}(\omega)\right]^{2} \times L^{2}(\omega)}$ from above, we can use Young's inequality such that

$$
\begin{aligned}
\|\mathcal{V}\|_{D_{I}}^{2} & =\left\|\mathcal{V}_{3}\right\|_{L^{2}(\omega)}^{2}=\int_{\omega} \mathcal{V}_{3}^{2} \mathrm{~d} s^{\prime} \\
& \leq \int_{\omega}\left[\left(\frac{\partial \mathcal{V}_{1}}{\partial s_{1}}\right)^{2}+\frac{1}{a^{2}} \mathcal{V}_{3}^{2}+\left(\frac{\partial \mathcal{V}_{2}}{\partial s_{2}}\right)^{2}+\left(\frac{\partial \mathcal{V}_{1}}{\partial s_{2}}\right)^{2}+\left(\frac{\partial \mathcal{V}_{2}}{\partial s_{1}}\right)+\mathcal{V}_{3}^{2}\right] \mathrm{d} s^{\prime} \\
& \leq C\left(\left\|\mathcal{V}_{1}\right\|_{H^{1}(\omega)}^{2}+\left\|\mathcal{V}_{2}\right\|_{H^{1}(\omega)}^{2}+\left\|\mathcal{V}_{3}\right\|_{L^{2}(\omega)}^{2}\right)=C\|\mathcal{V}\|_{\left[H^{1}(\omega)\right]^{2} \times L^{2}(\omega)}^{2}
\end{aligned}
$$

Step 2. We prove the inequalities.

With expression (5.1) 3 we get

$$
\begin{aligned}
\|\mathcal{V}\|_{D_{I}}^{2} & =\left\|\mathcal{V}_{3}\right\|_{L^{2}(\omega)}^{2}=\int_{\omega} \mathcal{V}_{3}^{2} \mathrm{~d} s_{1} \mathrm{~d} s_{2} \\
& =a^{2} \int_{0}^{a \pi} \int_{0}^{l}\left[\left(s_{2}^{c} V_{2}^{\prime \prime}\left(s_{1}\right)\right)^{2}-2 s_{2}^{c} V_{2}^{\prime \prime}\left(s_{1}\right) V_{1}^{\prime}\left(s_{1}\right)+\left(V_{1}^{\prime}\left(s_{1}\right)\right)^{2}\right] \mathrm{d} s_{2} \mathrm{~d} s_{1} \\
& \leq C\left(\left\|V_{2}^{\prime \prime}\right\|_{L^{2}(0, a \pi)}^{2}+\left\|V_{1}^{\prime}\right\|_{L^{2}(0, a \pi)}^{2}\right) .
\end{aligned}
$$

First we note that $V_{2}\left(s_{1}\right)=V_{2}^{\prime}\left(s_{1}\right)=0$ for $s_{1} \in\{0, a \pi\}$, which follows by the expressions in (5.1) and since $\mathcal{V}_{1}\left(0, s_{2}\right)=\mathcal{V}_{1}\left(a \pi, s_{2}\right)=\mathcal{V}_{2}\left(0, s_{2}\right)=\mathcal{V}_{2}\left(a \pi, s_{2}\right)=0$ for a.e. $s_{2} \in(0, l)$. Moreover, we obtain with the Poincaré inequality in $H_{0}^{1}(0, a \pi)$ and $H_{0}^{2}(0, a \pi)$ that,

$$
\begin{aligned}
\left\|V_{2}\right\|_{H^{2}(0, a \pi)}^{2}+\left\|V_{1}\right\|_{H^{1}(0, a \pi)}^{2} & \leq C\left(\left\|V_{2}^{\prime \prime}\right\|_{L^{2}(0, a \pi)}^{2}+\left\|V_{1}^{\prime}\right\|_{L^{2}(0, a \pi)}^{2}\right) \\
& \leq C \int_{\omega}\left(\left(s_{2}^{c} V_{2}^{\prime \prime}\right)^{2}-2 s_{2}^{c} V_{2}^{\prime \prime} V_{1}^{\prime}+\left(V_{1}^{\prime}\right)^{2}\right) \mathrm{d} s^{\prime} \\
& \leq C \int_{\omega} a^{2}\left(s_{2}^{c} V_{2}^{\prime \prime}-V_{1}^{\prime}\right)^{2} \mathrm{~d} s^{\prime}=C\|\mathcal{V}\|_{D_{I}}^{2} .
\end{aligned}
$$

The second inequality is again obtained in a similar way, where we need to use that

$$
\left\|V_{2}\right\|_{H^{4}(0, a \pi)}^{2}+\left\|V_{1}\right\|_{H^{3}(0, a \pi)}^{2} \leq C\left(\left\|V_{2}^{\prime \prime}\right\|_{H^{2}(0, a \pi)}^{2}+\left\|V_{1}^{\prime}\right\|_{H^{2}(0, a \pi)}^{2}\right),
$$

which follows from the Poincaré inequality. 
Proof of Lemma 5.2. Since $\mathbb{D}_{E}$ is the completion of $D_{E}$ for the norm $\|\cdot\|_{E}$, if we prove the estimates of the lemma for $\mathcal{U} \in D_{E}$, then by density they will be satisfied for every element in $\mathbb{D}_{E}$.

Let $\mathcal{U}$ be in $D_{E}$, recall that

$$
\left\|\frac{\partial \mathcal{U}_{1}}{\partial s_{1}}+\frac{1}{a} \mathcal{U}_{3}\right\|_{L^{2}(\omega)} \leq\|\mathcal{U}\|_{E}, \quad\left\|\frac{\partial \mathcal{U}_{2}}{\partial s_{2}}\right\|_{L^{2}(\omega)} \leq\|\mathcal{U}\|_{E}, \quad\left\|\frac{\partial \mathcal{U}_{1}}{\partial s_{2}}+\frac{\partial \mathcal{U}_{2}}{\partial s_{1}}\right\|_{L^{2}(\omega)} \leq\|\mathcal{U}\|_{E}
$$

Recall also that there exists $\left(C_{1}, C_{2}, C_{3}\right) \in \mathbb{R}^{3}$ such that

$$
\mathcal{M}_{2}\left(\mathcal{U}_{3}\right)\left(s_{1}\right)=C_{1}, \quad \mathcal{M}_{2}^{c}\left(\mathcal{U}_{3}\right)\left(s_{1}\right)=C_{2} s_{1}^{c}+C_{3}, \quad \text { for } \quad \text { a.e. } \quad s_{1} \in(0, a \pi) .
$$

Step 1. In this step we prove

$$
\begin{aligned}
\left\|\mathcal{M}_{2}\left(\mathcal{U}_{1}\right)\right\|_{H^{1}(0, a \pi)} & +\left\|\mathcal{M}_{2}^{c}\left(\mathcal{U}_{1}\right)\right\|_{H^{1}(0, a \pi)}+\left\|\mathcal{M}_{2}\left(\mathcal{U}_{2}\right)\right\|_{L^{2}(0, a \pi)} \\
& +\left|\mathcal{M}_{2}\left(\mathcal{U}_{3}\right)\right|+\left\|\mathcal{M}_{2}^{c}\left(\mathcal{U}_{3}\right)\right\|_{L^{2}(0, a \pi)} \leq C\|\mathcal{U}\|_{E} .
\end{aligned}
$$

Set

$$
\begin{aligned}
\widetilde{\mathcal{M}}_{2}^{c}\left(\mathcal{U}_{3}\right)\left(s_{1}\right) & =C_{2} \frac{s_{1}\left(s_{1}-a \pi\right)}{2}, \\
\mathcal{M}_{22}\left(\mathcal{U}_{2}\right)\left(s_{1}\right) & =\frac{1}{l} \int_{0}^{l} \mathcal{U}_{2}\left(s_{1}, s_{2}\right) \frac{s_{2}\left(s_{2}-l\right)}{2} \mathrm{~d} s_{2} .
\end{aligned}
$$

One has $\widetilde{\mathcal{M}}_{2}^{c}\left(\mathcal{U}_{3}\right), \mathcal{M}_{22}\left(\mathcal{U}_{2}\right) \in H_{0}^{1}(0, a \pi)$.

We first show that

$$
\left\|\frac{\mathrm{d} \mathcal{M}_{2}\left(\mathcal{U}_{1}\right)}{\mathrm{d} s_{1}}+\frac{1}{a} \mathcal{M}_{2}\left(\mathcal{U}_{3}\right)\right\|_{L^{2}(0, a \pi)} \leq \frac{1}{\sqrt{l}}\|\mathcal{U}\|_{E} .
$$

By plugging in the definition for $\mathcal{M}_{2}\left(\mathcal{U}_{i}\right)$ we get

$$
\left\|\frac{1}{l} \frac{\mathrm{d}}{\mathrm{d} s_{1}} \int_{0}^{l} \mathcal{U}_{1}\left(s_{1}, s_{2}\right) \mathrm{d} s_{2}+\frac{1}{a l} \int_{0}^{l} \mathcal{U}_{3}\left(s_{1}, s_{2}\right) \mathrm{d} s_{2}\right\|_{L^{2}(0, a \pi)} .
$$

We interchange differentiation and integration, s.t. with Jensen

$$
\left\|\frac{1}{l} \int_{0}^{l}\left(\frac{\partial \mathcal{U}_{1}}{\partial s_{1}}+\frac{1}{a} \mathcal{U}_{3}\right) \mathrm{d} s_{2}\right\|_{L^{2}(0, a \pi)} \leq \frac{1}{\sqrt{l}}\left\|\frac{\partial \mathcal{U}_{1}}{\partial s_{1}}+\frac{1}{a} \mathcal{U}_{3}\right\|_{L^{2}(\omega)} \leq \frac{1}{\sqrt{l}}\|\mathcal{U}\|_{E} .
$$

Moreover, we have

$$
\begin{aligned}
\left\|\frac{\mathrm{d} \mathcal{M}_{2}\left(\mathcal{U}_{1}\right)}{\mathrm{d} s_{1}}\right\|_{L^{2}(0, a \pi)}^{2}+\left\|\frac{1}{a} \mathcal{M}_{2}\left(\mathcal{U}_{3}\right)\right\|_{L^{2}(0, a \pi)}^{2} & =\int_{0}^{a \pi}\left(\frac{\mathrm{d} \mathcal{M}_{2}\left(\mathcal{U}_{1}\right)}{\mathrm{d} s_{1}}+\frac{1}{a} \mathcal{M}_{2}\left(\mathcal{U}_{3}\right)\right)^{2} \mathrm{~d} s_{1} \\
& =\left\|\frac{\mathrm{d} \mathcal{M}_{2}\left(\mathcal{U}_{1}\right)}{\mathrm{d} s_{1}}+\frac{1}{a} \mathcal{M}_{2}\left(\mathcal{U}_{3}\right)\right\|_{L^{2}(0, a \pi)}^{2}
\end{aligned}
$$

since by partial integration

$$
\int_{0}^{a \pi} \frac{\mathrm{d} \mathcal{M}_{2}\left(\mathcal{U}_{1}\right)}{\mathrm{d} s_{1}} \mathcal{M}_{2}\left(\mathcal{U}_{3}\right) \mathrm{d} s_{1}=\left[\mathcal{M}_{2}\left(\mathcal{U}_{1}\right) \mathcal{M}_{2}\left(\mathcal{U}_{3}\right)\right]_{0}^{a \pi}-\int_{0}^{a \pi} \mathcal{M}_{2}\left(\mathcal{U}_{1}\right) \frac{\mathrm{d} \mathcal{M}_{2}\left(\mathcal{U}_{3}\right)}{\mathrm{d}} s_{1} \mathrm{~d} s_{1}=0
$$

Therefore, we obtain

$$
\left\|\frac{\mathrm{d} \mathcal{M}_{2}\left(\mathcal{U}_{1}\right)}{\mathrm{d} s_{1}}\right\|_{L^{2}(0, a \pi)}^{2}+\left\|\frac{1}{a} \mathcal{M}_{2}\left(\mathcal{U}_{3}\right)\right\|_{L^{2}(0, a \pi)}^{2} \leq C\|\mathcal{U}\|_{E}^{2}
$$


The Poincaré inequality in $H_{0}^{1}(0, a \pi)$ and the previous results lead to

$$
\left\|\mathcal{M}_{2}\left(\mathcal{U}_{1}\right)\right\|_{H^{1}(0, a \pi)} \leq C\left\|\frac{\mathrm{d} \mathcal{M}_{2}\left(\mathcal{U}_{1}\right)}{\mathrm{d} s_{1}}\right\|_{L^{2}(0, a \pi)} \leq C\|\mathcal{U}\|_{E}
$$

and since $\mathcal{M}_{2}\left(\mathcal{U}_{3}\right)$ is independent of $s_{1}$ we obtain $\left|\mathcal{M}_{2}\left(\mathcal{U}_{3}\right)\right| \leq C\|\mathcal{U}\|_{E}$. Below we show the inequality

$$
\left\|\frac{\mathrm{d} \mathcal{M}_{2}^{c}\left(\mathcal{U}_{1}\right)}{\mathrm{d} s_{1}}+\frac{1}{a} \frac{\mathrm{d} \widetilde{\mathcal{M}}_{2}^{c}\left(\mathcal{U}_{3}\right)}{\mathrm{d} s_{1}}+\frac{C_{3}}{a}\right\|_{L^{2}(0, a \pi)} \leq C\|\mathcal{U}\|_{E} .
$$

Plugging in the definition for $\mathcal{M}_{2}^{c}$ and $\widetilde{\mathcal{M}}_{2}^{c}$ we get again with Jensen and since $s_{2}^{c} \in(-l / 2, l / 2)$

$$
\begin{aligned}
& \left\|\frac{\mathrm{d} \mathcal{M}_{2}^{c}\left(\mathcal{U}_{1}\right)}{\mathrm{d} s_{1}}+\frac{1}{a} \frac{\mathrm{d} \widetilde{\mathcal{M}}_{2}^{c}\left(\mathcal{U}_{3}\right)}{\mathrm{d} s_{1}}+\frac{C_{3}}{a}\right\|_{L^{2}(0, a \pi)} \\
& \quad=\left\|\frac{1}{l} \int_{0}^{l}\left(\frac{\partial \mathcal{U}_{1}}{\partial s_{1}}+\frac{1}{a} \mathcal{U}_{3}\right) s_{2}^{c} \mathrm{~d} s_{2}\right\|_{L^{2}(0, a \pi)} \leq\left\|\frac{1}{\sqrt{l}}\left(\frac{\partial \mathcal{U}_{1}}{\partial s_{1}}+\frac{1}{a} \mathcal{U}_{3}\right) s_{2}^{c}\right\|_{L^{2}(\omega)} \leq C\|\mathcal{U}\|_{E} \cdot
\end{aligned}
$$

Now, we prove the inequality

$$
\left\|\mathcal{M}_{2}^{c}\left(\mathcal{U}_{1}\right)+\frac{1}{a} \widetilde{\mathcal{M}}_{2}^{c}\left(\mathcal{U}_{3}\right)\right\|_{L^{2}(0, a \pi)}^{2}+\left|C_{3}\right|^{2} \leq C\|\mathcal{U}\|_{E}^{2} .
$$

With the Poincaré inequality and since $C_{3} \in \mathbb{R}$ and $\mathcal{M}_{2}^{c}\left(\mathcal{U}_{1}\right), \widetilde{\mathcal{M}}_{2}^{c}\left(\mathcal{U}_{3}\right) \in H_{0}^{1}(0, a \pi)$ we obtain

$$
\begin{aligned}
& \left\|\mathcal{M}_{2}^{c}\left(\mathcal{U}_{1}\right)+\frac{1}{a} \widetilde{\mathcal{M}}_{2}^{c}\left(\mathcal{U}_{3}\right)\right\|_{L^{2}(0, a \pi)}^{2}+\left|C_{3}\right|^{2} \\
& \quad \leq C\left(\left\|\frac{\mathrm{d}}{\mathrm{d} s_{1}} \mathcal{M}_{2}^{c}\left(\mathcal{U}_{1}\right)+\frac{1}{a} \frac{\mathrm{d}}{\mathrm{d} s_{1}} \widetilde{\mathcal{M}}_{2}^{c}\left(\mathcal{U}_{3}\right)\right\|_{L^{2}(0, a \pi)}^{2}+\left\|\frac{C_{3}}{a}\right\|_{L^{2}(0, a \pi)}^{2}\right) \leq C\|\mathcal{U}\|_{E},
\end{aligned}
$$

using

$$
\int_{0}^{a \pi}\left(\frac{\mathrm{d}}{\mathrm{d} s_{1}} \mathcal{M}_{2}^{c}\left(\mathcal{U}_{1}\right)+\frac{1}{a} \frac{\mathrm{d}}{\mathrm{d} s_{1}} \widetilde{\mathcal{M}}_{2}^{c}\left(\mathcal{U}_{3}\right)\right) \frac{C_{3}}{a} \mathrm{~d} s_{1}=0 .
$$

In the following we show the inequality

$$
\left\|\frac{\mathrm{d} \mathcal{M}_{22}\left(\mathcal{U}_{2}\right)}{\mathrm{d} s_{1}}+\frac{1}{a} \widetilde{\mathcal{M}}_{2}^{c}\left(\mathcal{U}_{3}\right)\right\|_{L^{2}(0, a \pi)} \leq C\|\mathcal{U}\|_{E} .
$$

With the previous result, partial integration and (A.11) we get

$$
\begin{aligned}
& \left\|\frac{\mathrm{d} \mathcal{M}_{22}\left(\mathcal{U}_{2}\right)}{\mathrm{d} s_{1}}+\frac{1}{a} \widetilde{\mathcal{M}}_{2}^{c}\left(\mathcal{U}_{3}\right)\right\|_{L^{2}(0, a \pi)} \\
& \quad=\left\|\frac{\mathrm{d} \mathcal{M}_{22}\left(\mathcal{U}_{2}\right)}{\mathrm{d} s_{1}}-\mathcal{M}_{2}^{c}\left(\mathcal{U}_{1}\right)+\mathcal{M}_{2}^{c}\left(\mathcal{U}_{1}\right)+\frac{1}{a} \widetilde{\mathcal{M}}_{2}^{c}\left(\mathcal{U}_{3}\right)\right\| \|_{L^{2}(0, a \pi)} \\
& \quad \leq\left\|\frac{\mathrm{d} \mathcal{M}_{22}\left(\mathcal{U}_{2}\right)}{\mathrm{d} s_{1}}-\mathcal{M}_{2}^{c}\left(\mathcal{U}_{1}\right)\right\|+\left\|\mathcal{M}_{2}^{c}\left(\mathcal{U}_{1}\right)+\frac{1}{a} \widetilde{\mathcal{M}}_{2}^{c}\left(\mathcal{U}_{3}\right)\right\|_{L^{2}(0, a \pi)}
\end{aligned}
$$

where we have for the first term by plugging in the definition and swapping integration with differentiation

$$
\begin{aligned}
& \left\|\frac{1}{l} \int_{0}^{l} \frac{\partial \mathcal{U}_{2}}{\partial s_{1}} \frac{s_{2}\left(s_{2}-l\right)}{2} \mathrm{~d} s_{2}-\frac{1}{l} \int_{0}^{l} \mathcal{U}_{1}\left(\cdot, s_{2}\right)\left(s_{2}-\frac{l}{2}\right) \mathrm{d} s_{2}\right\|_{L^{2}(0, a \pi)} \\
& \quad=\left\|\frac{1}{l} \int_{0}^{l}\left(\frac{\partial \mathcal{U}_{2}}{\partial s_{1}}+\frac{\partial \mathcal{U}_{1}}{\partial s_{2}}\right) \frac{s_{2}\left(s_{2}-l\right)}{2} \mathrm{~d} s_{2}\right\|_{L^{2}(0, a \pi)} \leq C\left\|\frac{\partial \mathcal{U}_{2}}{\partial s_{1}}+\frac{\partial \mathcal{U}_{1}}{\partial s_{2}}\right\|_{L^{2}(\omega)} \leq C\|\mathcal{U}\|_{E} \cdot
\end{aligned}
$$


Integrating $\frac{\mathrm{d} \mathcal{M}_{22}\left(\mathcal{U}_{2}\right)}{\mathrm{d} s_{1}}+\frac{1}{a} \widetilde{\mathcal{M}}_{2}^{c}\left(\mathcal{U}_{3}\right)$ over $(0, a \pi)$ and due to the above estimate (A.16), one obtains $\left|C_{2}\right| \leq$ $C\|\mathcal{U}\|_{E}$ and then again with (A.16) and (A.14), (A.15)

$$
\left\|\widetilde{\mathcal{M}}_{2}^{c}\left(\mathcal{U}_{3}\right)\right\|_{L^{2}(0, a \pi)}+\left\|\mathcal{M}_{2}^{c}\left(\mathcal{U}_{3}\right)\right\|_{L^{2}(0, a \pi)}+\left\|\mathcal{M}_{2}^{c}\left(\mathcal{U}_{1}\right)\right\|_{H^{1}(0, a \pi)} \leq C\|\mathcal{U}\|_{E}+\left\|\frac{\mathrm{d} \mathcal{M}_{22}\left(\mathcal{U}_{2}\right)}{\mathrm{d} s_{1}}\right\|_{L^{2}(0, a \pi)} \leq C\|\mathcal{U}\|_{E} .
$$

Since $\mathcal{M}_{22}\left(\mathcal{U}_{2}\right) \in H_{0}^{1}(0, a \pi),\left\|\mathcal{M}_{22}\left(\mathcal{U}_{2}\right)\right\|_{L^{2}(0, a \pi)} \leq C\left\|\frac{\mathrm{d} \mathcal{M}_{22}\left(\mathcal{U}_{2}\right)}{\mathrm{d} s_{1}}\right\|_{L^{2}(0, a \pi)} \leq C\|\mathcal{U}\|_{E}$. The PoincaréWirtinger inequality gives

$$
\left\|\mathcal{U}_{2}-\mathcal{M}_{2}\left(\mathcal{U}_{2}\right)\right\|_{L^{2}(\omega)} \leq C\|\mathcal{U}\|_{E}
$$

Multiply $\mathcal{U}_{2}-\mathcal{M}_{2}\left(\mathcal{U}_{2}\right)$ by $\frac{s_{2}\left(s_{2}-l\right)}{2}$ and then integrate with respect to $s_{2}$ to get

$$
\left\|\mathcal{M}_{22}\left(\mathcal{U}_{2}\right)+\frac{l^{2}}{12} \mathcal{M}_{2}\left(\mathcal{U}_{2}\right)\right\|_{L^{2}(0, a \pi)} \leq C\|\mathcal{U}\|_{E}
$$

Therefore

$$
\left\|\mathcal{M}_{2}\left(\mathcal{U}_{2}\right)\right\|_{L^{2}(0, a \pi)} \leq C\|\mathcal{U}\|_{E}
$$

Step 2. We show the 3 inequalities in equation (5.3) by using Poincaré-Wirtinger inequality. We start with $\left\|\mathcal{U}_{2}\right\|_{L^{2}(\omega)} \leq C\|\mathcal{U}\|_{E}$. With the inequalities in (A.17) and (A.18) we get

$$
\left\|\mathcal{U}_{2}\right\|_{L^{2}(\omega)} \leq\left\|\mathcal{U}_{2}-\mathcal{M}_{2}\left(\mathcal{U}_{2}\right)\right\|_{L^{2}(\omega)}+\left\|\mathcal{M}_{2}\left(\mathcal{U}_{2}\right)\right\|_{L^{2}(\omega)} \leq C\|\mathcal{U}\|_{E}
$$

Recall that if $X$ is a separable Hilbert space, then the Poincaré-Wirtinger inequality is valid in $W^{1, p}(0, l ; X)$ $(p \in[1,+\infty])$. From $($ A.19) and (A.11) 3 we get

$$
\left\|\frac{\partial \mathcal{U}_{2}}{\partial s_{1}}\right\|_{L^{2}\left(0, l ;\left(H^{1}(0, a \pi)\right)^{\prime}\right)}+\left\|\frac{\partial \mathcal{U}_{1}}{\partial s_{2}}\right\|_{L^{2}\left(0, l ;\left(H^{1}(0, a \pi)\right)^{\prime}\right)} \leq C\|\mathcal{U}\|_{E}
$$

Then the Poincaré-Wirtinger inequality and estimate $(\mathrm{A} .12)_{1}$ in $H^{1}\left(0, l ;\left(H^{1}(0, a \pi)\right)^{\prime}\right)$ give

$$
\left\|\mathcal{U}_{1}\right\|_{L^{2}\left(0, l ;\left(H^{1}(0, a \pi)\right)^{\prime}\right)} \leq\left\|\mathcal{U}_{1}-\mathcal{M}_{2}\left(\mathcal{U}_{1}\right)\right\|_{L^{2}\left(0, l ;\left(H^{1}(0, a \pi)\right)^{\prime}\right)}+\left\|\mathcal{M}_{2}\left(\mathcal{U}_{1}\right)\right\|_{L^{2}\left(0, l ;\left(H^{1}(0, a \pi)\right)^{\prime}\right)} \leq C\|\mathcal{U}\|_{E}
$$

The above inequality leads to $\left\|\frac{\partial \mathcal{U}_{1}}{\partial s_{1}}\right\|_{L^{2}\left(0, l ;\left(H^{2}(0, a \pi)\right)^{\prime}\right)} \leq C\|\mathcal{U}\|_{E}$, which together with $($ A.11) 1 yields

$$
\left\|\mathcal{U}_{3}\right\|_{L^{2}\left(0, l ;\left(H^{2}(0, a \pi)\right)^{\prime}\right)} \leq C\|\mathcal{U}\|_{E} .
$$

This ends the proof of the lemma.

\section{REFERENCES}

[1] A. Bensoussan, J.-L. Lions and G. Papanicolaou, Asymptotic Analysis for Periodic Structures. In: Vol. 5 of Studies in Mathematics and its Applications. North Holland (1978).

[2] M. Bernadou, P.G. Ciarlet and B. Miara, Existence theorems for two-dimensional linear shell theories. J. Elast. 34 (1994) $111-138$.

[3] D. Blanchard and G. Griso, Decomposition of the deformations of a thin shell. Asymptotic behavior of the Green-St Venant's strain tensor. J. Elast. 101 (2010) 179-205.

[4] D. Blanchard and G. Griso, Decomposition of deformations of thin rods: application to nonlinear elasticity. Anal. Appl. 7 (2009) 21-71.

[5] D. Caillerie, Thin elastic and periodic plates. Math. Methods Appl. Sci. 6 (1984) 159-191. 
[6] P.G. Ciarlet, Mathematical Elasticity. Vol. III. Theory of shells. In: Vol. 29 of Studies in Mathematics and its Applications. North-Holland (2000).

[7] P.G. Ciarlet and V. Lods, Asymptotic analysis of linearly elastic shells. I. Justification of membrane shell equations. Arch. Ratio. Mech. Anal. 136 (1996) 119-161.

[8] P.G. Ciarlet and B. Miara, On the ellipticity of linear shell models. Z. Angew. Math. Phys. 43 (1992) $243-253$.

[9] D. Cioranescu, A. Damlamian and G. Griso, The periodic unfolding method in homogenization. SIAM J. Math. Anal. 40 (2008) 1585-1620.

[10] D. Cioranescu, A. Damlamian and G. Griso, The Periodic Unfolding Method: Theory and Applications to Partial Differential Problems. Vol. 3 of Series in Contemporary Mathematics. Springer, Singapore (2018).

[11] E. De Giorgi and S. Spagnolo, Sulla convergenza degli integrali dell'energia per operatori ellittici del secondo ordine. Boll. Un. Mat. Ital. 4 (1973) 391-411.

[12] M. Ghergu, G. Griso, H. Mechkour and B. Miara, Homogenization of thin piezoelectricperforated shells. ESAIM: M2AN 41 (2007) 875-895.

[13] G. Griso, Asymptotic behaviour of curved rods by the unfolding method. Math. Methods Appl. Sci. 27 (2004) $2081-2110$.

[14] G. Griso, Decompositions of displacements of thin structures. J. Math. Pure Appl. 89 (2008) 199-223.

[15] G. Griso and B. Miara, Homogenization of periodically heterogeneous thin beams. Chin. Ann. Math. Ser. B 39 (2018) $397-426$.

[16] G. Griso, J. Orlik and S. Wackerle, Asymptotic behavior for textiles in von-Kármán regime (to appear in JMPA). Preprint arXiv:1912.10928 (2019).

[17] B. Miara and V. Valente, Exact controllability of a Koiter shell by a boundary action. J. Elast. 52 (1998/1999) 267-287.

[18] G. Nguetseng, A general convergence result for a functional related to the theory of homogenization. SIAM J. Math. Anal. 20 (1989) 608-629.

[19] M. Neuss-Radu, Mathematical modelling and multi-scale analysis of transport processes through membranes, Diss. Habilitation thesis. University of Heidelberg (2017).

[20] G. Panasenko, Multi-Scale Modelling for Structures and Composites. Springer, Dordrecht (2005).

[21] E. Sanchez-Palencia, Non Homogeneous Media and Vibration Theory. In: Lecture Notes in Physics. Springer, Berlin-Heidelberg (1980).

[22] K. Yosida, Functional Analysis. Springer, Berlin-Heidelberg (1980).

[23] E. Zeidler, Nonlinear Functional Analysis and its Applications. II/B. Nonlinear Monotone Operators. Translated from the German by the author and Leo F. Boron. Springer, New York, NY (1990). 